\title{
The calculus of Volterra Mellin pseudodifferential operators with operator-valued symbols
}

\author{
Thomas Krainer ${ }^{1}$ \\ Institut für Mathematik \\ Universität Potsdam \\ Postfach 601553 \\ D-14415 Potsdam \\ Germany \\ krainer@math.uni-potsdam.de
}

December 14, 2001

\begin{abstract}
We introduce the calculus of Mellin pseudodifferential operators with parameters based on "twisted" operator-valued Volterra symbols as well as the abstract Mellin calculus with holomorphic symbols. We establish the properties of the symbolic and operational calculi, and we give and make use of explicit oscillatory integral formulas on the symbolic side, e. g., for the Leibniz-product, kernel cut-off, and Mellin quantization.

Moreover, we introduce the notion of parabolicity for the calculi of Volterra Mellin operators, and construct Volterra parametrices for parabolic operators within the calculi.
\end{abstract}

Mathematics Subject Classification (2000): 35S05 (Primary) 35S15, 35K40, 35K50, 35K65 (Secondary)

\section{Contents}

\section{Introduction}

1 Basic notation and general conventions 5

1.1 Sets of real and complex numbers . . . . . . . . . . . . 5

1.2 Multi-index notation .................. 6

1.3 Functional analysis and basic function spaces . . . . . . . . . 7

1.4 Tempered distributions and the Fourier transform . . . . . . . 8

2 Preliminaries on function spaces and the Mellin transform 8

2.1 A Paley-Wiener type theorem . . . . . . . . . . . . . 10

2.2 The Mellin transform in distributions . . . . . . . . . . . . 10

\footnotetext{
${ }^{1}$ Supported by the Deutsche Forschungsgemeinschaft
} 
3 The calculus of Volterra symbols $\quad 11$

3.1 General anisotropic parameter-dependent symbols . . . . . . . . . 11

3.1.1 Asymptotic expansion . . . . . . . . . . . . . . . . 14

3.2 Homogeneity and classical symbols . . . . . . . . . . . . . . . . 14

3.3 Parameter-dependent Volterra symbols . . . . . . . . . . . . . . . 17

3.3.1 The translation operator in Volterra symbols . . . . . . . . 19

3.4 Holomorphic Volterra symbols . . . . . . . . . . . . . . . . . . 21

4 The calculus of Volterra Mellin operators 24

4.1 General Volterra Mellin operators . . . . . . . . . . . . . . . . . 24

4.2 Continuity in Mellin Sobolev spaces . . . . . . . . . . . . 33

4.3 Volterra Mellin operators with analytic symbols . . . . . . . . . 35

5 Kernel cut-off and Mellin quantization $\quad 40$

5.1 The Mellin kernel cut-off operator . . . . . . . . . . . . . . 40

5.2 Degenerate symbols and Mellin quantization . . . . . . . . . . 42

6 Parabolicity and Volterra parametrices 49

6.1 Ellipticity and parabolicity on symbolic level . . . . . . . . . . 49

6.2 The parametrix construction ............. 53

References

\section{Introduction}

The present article contributes to the axiomatic framework of the analysis of degenerate partial differential equations, e. g., of PDEs on manifolds with singularities, with pseudodifferential methods.

Substantial progress has been achieved in recent years, in particular, in the theory of degenerate elliptic equations. The general concept is to embed the natural systems of elliptic partial differential equations into a suitable calculus of pseudodifferential operators that admits the construction of parametrices of elliptic elements within, and to study the qualitative properties of the equations such as regularity and asymptotics of solutions and the Fredholm property via algebraic methods on side of the operator algebra, see, e. g., Schulze [40], [43], [44].

Just recently, this concept has been further developed also in the study of parabolic equations, see, e. g., Buchholz and Schulze [6], and Krainer [24], Krainer and Schulze [25], [26]. More precisely, the natural systems of parabolic partial differential equations are embedded in a suitable calculus of pseudodifferential operators that admits the construction of inverses of parabolic elements. In particular, in addition to the elliptic theory, the existence and uniqueness of solutions follow, and, via analyzing the operator and symbolic structure of the calculus, insights about 
the qualitative properties of the equations and the structure of solution operators are obtained.

A typical feature of the parabolic theory is that the pseudodifferential operators have the so-called Volterra property with respect to time, i. e., the (anisotropic) symbols extend holomorphically in the time covariable to a complex half-plane, including the symbol estimates. The classical calculus of such operators was introduced by Piriou [30], [31] in the study of parabolic pseudodifferential equations on a finite time interval, and a closed (compact) spatial manifold.

The (pseudodifferential) analysis of (degenerate) partial differential equations on manifolds with singularities and boundary value problems encompasses, in particular, the crucial task to describe the behaviour of the equations close to the singular sets and the boundary, where, typically, extra conditions of trace and potential type are involved that are associated with the operators in a natural way. In this context, the abstract theory of pseudodifferential calculus with "twisted" operator-valued symbols was introduced by Schulze (see, e. g., [38], [40]) in order to describe the general structure of these conditions, as well as the structure of the operators and the singular Green remainders as they have to be (re)formulated with respect to a given splitting of coordinates on and transversal to wedges or boundaries.

Several authors have contributed since to the pseudodifferential calculus with operator-valued symbols for it provides a general axiomatic framework for the pseudodifferential analysis of degenerate partial differential equations and boundary value problems, i. e., in concrete situations such as the calculus on manifolds with conical singularities, edges, and corners, many functional analytic properties can be traced back to the calculus of operators with operator-valued symbols; see, e. g., Behm [2], Dorschfeldt [7], Dorschfeldt, Grieme, and Schulze [8], Krainer [24], and Seiler [47]. Material about pseudodifferential calculus with anisotropic operator-valued symbols can be found in Buchholz and Schulze [6], Gil [13], and Krainer [24], and the theory of operators with operator-valued Volterra symbols has so far been considered in Buchholz [4], Buchholz and Schulze [6], and Krainer [23], [24].

The calculus of Mellin operators occurs, in particular, in the study of operators near conical points, or, more generally, corner singularities (see, e. g., Schulze [39], [40], [41]). The operators are formulated as Mellin operators with respect to the distance variable (and covariable) to the corner point, where the singularity is located at the origin. In order to handle the functional analytic structures for higher corners, there have been also investigations about abstract Mellin calculus with operator-valued symbols, see, e. g., Dorschfeldt [7], Dorschfeldt and Schulze [9]. Up to now, however, any considerations in the direction of Volterra Mellin operators seemed to be unnecessary.

Recently, in the pseudodifferential analysis of parabolic partial differential equations on an infinite time interval a connection has been drawn to the pseudodif- 
ferential analysis on manifolds with singularities via interpreting $t=\infty$ as an anisotropic conical or corner point for the infinite space-time configuration, and it has been shown that this interpretation in combination with the Volterra calculus furnishes the appropriate idea to study (degenerate) parabolic equations and their qualitative properties in infinite space-time, see Krainer [24], Krainer and Schulze [25], [26]. In this context, considerations about Mellin pseudodifferential calculus built upon operator-valued Volterra symbols are a necessary and natural step, and they provide the functional analytic background that is crucial to handle these problems.

The purpose of the present paper is twofold: On the one hand, the main results about Mellin operators with operator-valued symbols that are available in the literature so far are collected and extended in order to give a unified and efficient approach to the theory. To this end, we employ explicit oscillatory integral techniques and symbols that satisfy global estimates in the variables ("Kumano-go's technique"), and we formulate the theory with an extra (anisotropic) parameter $\xi \in \mathbb{R}^{n}$ that represents, heuristically, the presence of an $n$-dimensional covariable transversal to the Mellin covariable, which is the general setting in applications. On the other hand, we introduce the holomorphic Mellin calculus with operatorvalued symbols, as well as the subcalculi of Volterra Mellin operators. The direct approach via oscillatory integral techniques enables us to establish the symbolic and operational calculi in a transparent form, where, in particular, manipulations on both sides are considered separated from each other.

The text is organized as follows: In Section 1 we give an account on the basic notation and general conventions that are freely used throughout this work, while Section 2 is devoted to recall the definition and some elementary properties of the vector-valued (weighted) Mellin transform and associated function spaces.

Section 3 deals with the symbolic calculus of the classes of operator-valued (Volterra) symbols. We briefly recall the basic definitions and properties of general anisotropic symbols as well as the concept of homogeneity and classical symbols in Sections 3.1 and 3.2 before we enter the discussion of Volterra symbols and holomorphic (Volterra) symbols. The analyticity in the covariable represents the major difficulty in the symbolic calculus because arguments involving excision functions cannot be employed. Usually, excision functions are used, e. g., to establish the asymptotic completeness, i. e., that it is possible to find symbols having a prescribed asymptotic expansion, as well as to prove that the principal symbol sequence is exact; both aspects are important for the construction of parametrices. The analysis of the translation operator in Volterra symbols, and the kernel cut-off operator (see Section 5.1), provide the appropriate tools to overcome these difficulties.

In Section 4 we study the operational calculi of Mellin operators that are built upon operator-valued (Volterra) symbols, and we analyze how the manipulations on side of the operators are reflected on the symbolic side. The approach via direct 
oscillatory integral techniques furnishes explicit formulas that are used to give easy proofs that the calculus of Volterra operators and the holomorphic subcalculi are indeed closed with respect to the relevant operations, e. g., taking compositions.

Traditionally, the calculus of Mellin pseudodifferential operators has been used, e. g., in the boundary symbolic calculus, and, more generally, edge symbolic calculus (see Eskin [12], Schulze [40], [42], Gil, Schulze, and Seiler [14], [15]). More precisely, (Kohn-Nirenberg quantized) pseudodifferential operators with degenerate symbols are reformulated as Mellin operators modulo smoothing remainders in order to analyze the behaviour of the operators in weighted function spaces adapted to the singular structures. This reformulation is also known as Mellin quantization, and several authors have contributed to the task of constructing such operator conventions. We give in Section 5 (more precisely in Section 5.2) a direct Mellin quantization in terms of an oscillatory integral formula purely on symbolic level, and prove the isomorphism between Kohn-Nirenberg quantized pseudodifferential operators with degenerate operator-valued (Volterra) symbols and holomorphic (Volterra) Mellin pseudodifferential operators up to smoothing (Volterra) remainders. Moreover, in Section 5.1 we give an extended definition of the Mellin kernel cut-off operator in terms of a direct oscillatory integral formula and summarize the resulting properties. The kernel cut-off technique is an appropriate tool to prove the asymptotic completeness of the operator-valued Volterra symbol classes, see also Buchholz and Schulze [6]; a detailed discussion of the kernel cut-off technique (based on the Fourier transform) in operator-valued symbols can be found, e. g., in Krainer [23].

Finally, in Section 6, we recall the notion of ellipticity and introduce the notion of parabolicity for the Volterra symbol classes, and we give a proof of the existence of (Volterra) parametrices within the Mellin calculi. The parametrix construction is performed via symbolic inversion and the classical formal Neumann series argument, and the algebraic properties and results from the preceding sections are used, in particular, in the discussion of symbolic invertibility and asymptotic expansions.

Acknowledgement: The author expresses his gratitude to Professor B.-W. Schulze from the University of Potsdam for numerous scientific discussions, and for his encouragement to prepare the present article.

\section{Basic notation and general conventions}

\subsection{Sets of real and complex numbers}

- We denote:

$\mathbb{C}$ the complex numbers, 


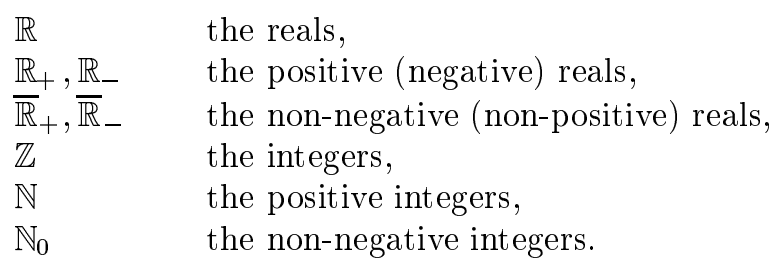

- Let $\mathbb{C}^{N}$ and $\mathbb{R}^{N}$ denote the complex $N$-space, respectively the Euclidean $N$ space, in the variables $\left(z_{1}, \ldots, z_{N}\right) \in \mathbb{C}^{N}$ or $\left(x_{1}, \ldots, x_{N}\right) \in \mathbb{R}^{N}$, respectively. In general, we allow $N$ to be zero, and in this case these spaces degenerate to the set containing a single point only.

- The upper and lower half-plane in $\mathbb{C}$ will be denoted as

$$
\begin{aligned}
\mathbb{H} & :=\{z \in \mathbb{C} ; \operatorname{Im}(z) \geq 0\}, \\
\mathbb{H}_{-}: & :=\{z \in \mathbb{C} ; \operatorname{Im}(z) \leq 0\},
\end{aligned}
$$

respectively. Moreover, for $\beta \in \mathbb{R}$ let

$$
\begin{aligned}
\Gamma_{\beta} & :=\{z \in \mathbb{C} ; \operatorname{Re}(z)=\beta\}, \\
\mathbb{H}_{\beta}: & =\{z \in \mathbb{C} ; \operatorname{Re}(z) \geq \beta\} .
\end{aligned}
$$

We refer to $\Gamma_{\beta}$ also as a weight line. With the splitting $z=\beta+i \tau$ into real and imaginary part we shall identify $\Gamma_{\beta}$ with $\mathbb{R}$ via $\Gamma_{\beta} \ni z=\beta+i \tau \leftrightarrow \tau \in \mathbb{R}$. Analogously, we have an identification of $\mathbb{H}_{\beta}$ with the right half-plane $\mathbb{H}_{0}$ via $\mathbb{H}_{\beta} \ni z=\beta+\zeta \leftrightarrow \zeta \in \mathbb{H}_{0}$, i. e., $\mathbb{H}_{\beta}$ originates from $\mathbb{H}_{0}$ via translation, and we shall also employ the identification of $\mathbb{H}_{\beta}$ with the upper half-plane $\mathbb{H}$ via $\mathbb{H} \ni \zeta \leftrightarrow \beta-i \zeta \in \mathbb{H}_{\beta}$.

- The Euclidean norm of $x=\left(x_{1}, \ldots, x_{N}\right) \in \mathbb{R}^{N}$ is denoted as $|x|=\left(\sum_{j=1}^{N} x_{j}^{2}\right)^{\frac{1}{2}}$. Moreover, let $\langle x\rangle=\left(1+|x|^{2}\right)^{\frac{1}{2}}$ be the standard regularized distance in $\mathbb{R}^{N}$. The inner product in $\mathbb{R}^{N}$ is denoted as $\langle x, \xi\rangle=x \xi=\sum_{j=1}^{N} x_{j} \xi_{j}$.

\subsection{Multi-index notation}

We employ the standard multi-index notation.

For multi-indices $\alpha=\left(\alpha_{1}, \ldots, \alpha_{N}\right), \beta=\left(\beta_{1}, \ldots, \beta_{N}\right) \in \mathbb{N}_{0}^{N}$ we denote

$$
\left(\begin{array}{l}
\alpha \\
\beta
\end{array}\right)=\left(\begin{array}{c}
\alpha_{1} \\
\beta_{1}
\end{array}\right) \ldots\left(\begin{array}{c}
\alpha_{N} \\
\beta_{N}
\end{array}\right) \quad \alpha !=\prod_{j=1}^{N} \alpha_{j} ! \quad|\alpha|=\sum_{j=1}^{N} \alpha_{j} .
$$


We write $\alpha \leq \beta$ if the inequality holds componentwise. Moreover, (normalized) partial derivatives with respect to the variables $x=\left(x_{1}, \ldots, x_{N}\right) \in \mathbb{R}^{N}$ are written as

$$
\partial_{x}^{\alpha}=\frac{\partial^{|\alpha|}}{\partial_{x_{1}}^{\alpha_{1}} \ldots \partial_{x_{N}}^{\alpha_{N}}} \quad D_{x}^{\alpha}=(-i)^{|\alpha|} \partial_{x}^{\alpha} .
$$

In case a function $f(x, \lambda)$ depends on the group of complex variables $\lambda \in \mathbb{C}^{M}$ we also use the notations

$$
\begin{array}{ll}
\partial_{\lambda}^{\beta} f=\frac{\partial^{|\beta|}}{\partial_{\lambda_{1}}^{\beta_{1}} \ldots \partial_{\lambda_{M}}^{\beta_{M}}} f & D_{\lambda}^{\beta} f=(-i)^{|\beta|} \partial_{\lambda}^{\beta} f, \\
\partial_{\bar{\lambda}}^{\beta} f=\frac{\partial^{|\beta|}}{\partial_{\bar{\lambda}_{1}}^{\beta_{1}} \ldots \partial_{\bar{\lambda}_{M}}^{\beta_{M}}} f & D_{\bar{\lambda}}^{\beta} f=(-i)^{|\beta|} \partial_{\bar{\lambda}}^{\beta} f .
\end{array}
$$

For $z=\left(z_{1}, \ldots, z_{N}\right) \in \mathbb{C}^{N}$ and $\alpha=\left(\alpha_{1}, \ldots, \alpha_{N}\right) \in \mathbb{N}_{0}^{N}$ we write $z^{\alpha}=z_{1}^{\alpha_{1}} \cdot \ldots \cdot z_{N}^{\alpha_{N}}$.

\subsection{Functional analysis and basic function spaces}

Unless stated explicitly otherwise, the spaces in this work are always assumed to be complex. For topological vector spaces $E$ and $F$ we denote the space of continuous linear operators $E \longrightarrow F$ as $\mathcal{L}(E, F)$. Moreover, the topological dual of $E$ is denoted as $E^{\prime}$. We write $E \otimes F$ for the algebraical tensor product of $E$ and $F$. The projective topology on $E \otimes F$ is indicated by the subscript $E \otimes_{\pi} F$, while $E \widehat{\otimes}_{\pi} F$ denotes the completion. We employ the notation $\langle\cdot, \cdot\rangle_{E, F}$, or just $\langle\cdot, \cdot\rangle$, when we deal with a duality $E \times F \longrightarrow \mathbb{C}$. The inner product in a Hilbert space $E$ is also denoted as $\langle\cdot, \cdot\rangle_{E}$, or simply as $\langle\cdot, \cdot\rangle$.

Moreover, we have the following spaces of $E$-valued functions on $M$ (where $M$ and $E$ are appropriate):

$\begin{array}{ll}L^{p}(M, E) & \text { measurable functions } u \text { with } \int_{M}\|u(x)\|_{E}^{p} d x<\infty \\ & \text { (with respect to Lebesgue measure, } 1 \leq p<\infty), \\ C(M, E) & \text { continuous functions, } \\ \mathcal{A}(M, E) & \text { analytic functions, } \\ C^{k}(M, E) & \text {-times continuously differentiable functions, } \\ C^{\infty}(M, E) & \text { smooth functions, } \\ C_{0}^{\infty}(M, E) & \text { smooth functions with compact support, } \\ C_{b}^{\infty}(M, E) & \text { smooth functions with bounded derivatives, } \\ \mathcal{S}(M, E) & \text { rapidly decreasing functions, } \\ \mathcal{D}^{\prime}(M, E)=\mathcal{L}\left(C_{0}^{\infty}(M), E\right) & \text { distributions, } \\ \mathcal{E}^{\prime}(M, E)=\mathcal{L}\left(C^{\infty}(M), E\right) & \text { distributions with compact support, } \\ \mathcal{S}^{\prime}(M, E)=\mathcal{L}(\mathcal{S}(M), E) & \text { tempered distributions. }\end{array}$


Let $E$ be a Fréchet space. Define

$$
C_{B}^{\infty}\left(\left(\mathbb{R}_{+}\right)^{q}, E\right):=\left\{u \in C^{\infty}\left(\left(\mathbb{R}_{+}\right)^{q}, E\right) ;\left(\left(-r \partial_{r}\right)^{k} u\right)\left(\left(\mathbb{R}_{+}\right)^{q}\right) \subseteq E\right.
$$

is bounded for all $\left.k \in \mathbb{N}_{0}^{q}\right\}$,

endowed with the Fréchet topology of uniform convergence of $\left(-r \partial_{r}\right)^{k} u$ on $\left(\mathbb{R}_{+}\right)^{q}$ for every $k \in \mathbb{N}_{0}^{q}$. Here we use the notation $\left(-r \partial_{r}\right)^{k}:=\left(-r_{1} \partial_{r_{1}}\right)^{k_{1}} \ldots\left(-r_{q} \partial_{r_{q}}\right)^{k_{q}}$ for $r=\left(r_{1}, \ldots, r_{q}\right) \in\left(\mathbb{R}_{+}\right)^{q}$ and $k=\left(k_{1}, \ldots, k_{q}\right) \in \mathbb{N}_{0}^{q}$.

Moreover, let $C_{B}^{\infty}\left(\left(\overline{\mathbb{R}}_{+}\right)^{q}, E\right):=C_{B}^{\infty}\left(\left(\mathbb{R}_{+}\right)^{q}, E\right) \cap C_{b}^{\infty}\left(\left(\mathbb{R}_{+}\right)^{q}, E\right)$ be the subspace of all functions that extend smoothly to $\left(\overline{\mathbb{R}}_{+}\right)^{q}$.

If $E=\mathbb{C}$ we drop it from the notation.

\subsection{Tempered distributions and the Fourier transform}

Let $E$ be a Hilbert space. Partial derivatives of a distribution $u \in \mathcal{S}^{\prime}\left(\mathbb{R}^{n}, E\right)$ are defined as $\left\langle\partial_{x}^{\alpha} u, \varphi\right\rangle=(-1)^{|\alpha|}\left\langle u, \partial_{x}^{\alpha} \varphi\right\rangle$, while multiplication with a function $\psi$ of tempered growth is given as $\langle\psi u, \varphi\rangle=\langle u, \psi \varphi\rangle$. A distribution $u \in \mathcal{S}^{\prime}\left(\mathbb{R}^{n}, E\right)$ is called regular, if $u$ is a Bochner measurable function, and there exists $N \in \mathbb{N}_{0}$ with $\int_{\mathbb{R}^{n}}\langle x\rangle^{-N}\|u(x)\|_{E} d x<\infty$. Note that we identify regular distributions with their densities. In this sense we in particular have $L^{p}\left(\mathbb{R}^{n}, E\right) \hookrightarrow \mathcal{S}^{\prime}\left(\mathbb{R}^{n}, E\right)$.

We employ the normalized Fourier transform $\mathcal{F}: \mathcal{S}\left(\mathbb{R}^{n}\right) \longrightarrow \mathcal{S}\left(\mathbb{R}^{n}\right)$, i. e.,

$$
\begin{aligned}
(\mathcal{F} u)(\xi) & =(2 \pi)^{-\frac{n}{2}} \int_{\mathbb{R}^{n}} e^{-i x \xi} u(x) d x, \\
\left(\mathcal{F}^{-1} u\right)(x) & =(2 \pi)^{-\frac{n}{2}} \int_{\mathbb{R}^{n}} e^{i x \xi} u(\xi) d \xi
\end{aligned}
$$

for $u \in \mathcal{S}\left(\mathbb{R}^{n}\right)$. For Fréchet spaces $E$ the Fourier transform extends to an isomorphism $\mathcal{S}\left(\mathbb{R}^{n}, E\right) \longrightarrow \mathcal{S}\left(\mathbb{R}^{n}, E\right)$ via $\mathcal{F}=\mathcal{F} \widehat{\otimes}_{\pi} \mathrm{id}_{E}$, noting that $\mathcal{S}\left(\mathbb{R}^{n}, E\right) \cong$ $\mathcal{S}\left(\mathbb{R}^{n}\right) \widehat{\otimes}_{\pi} E$. If $E$ is a Hilbert space we have $\mathcal{F}: \mathcal{S}^{\prime}\left(\mathbb{R}^{n}, E\right) \longrightarrow \mathcal{S}^{\prime}\left(\mathbb{R}^{n}, E\right)$ via $\langle\mathcal{F} u, \varphi\rangle=\langle u, \mathcal{F} \varphi\rangle$.

In oscillatory integral formulas, however, we shall follow the tradition and employ the normalized measure $d \xi=(2 \pi)^{-n} d \xi$ on the side of the covariables.

\section{Preliminaries on function spaces and the Mellin transform}

Let $E$ be a Fréchet space. 
- For $\gamma \in \mathbb{R}$ let

$$
\mathcal{T}_{\gamma}\left(\mathbb{R}_{+}, E\right):=\left\{u \in C^{\infty}\left(\mathbb{R}_{+}, E\right) ;\left(r^{\frac{1}{2}-\gamma}\langle\log (r)\rangle^{m}\left(-r \partial_{r}\right)^{k} u\right)\left(\mathbb{R}_{+}\right) \subseteq E\right.
$$

is bounded for all $\left.k, m \in \mathbb{N}_{0}\right\}$.

This space is endowed with the Fréchet topology of uniform convergence of $r^{\frac{1}{2}-\gamma}\langle\log (r)\rangle^{m}\left(-r \partial_{r}\right)^{k} u$ on $\mathbb{R}_{+}$for every $k, m \in \mathbb{N}_{0}$.

Note that for every $\delta \in \mathbb{R}$ the operator of multiplication with the function $r^{\delta}$ induces a topological isomorphism $r^{\delta}: \mathcal{T}_{\gamma}\left(\mathbb{R}_{+}, E\right) \longrightarrow \mathcal{T}_{\gamma+\delta}\left(\mathbb{R}_{+}, E\right)$.

- For $\gamma \in \mathbb{R}$ define the operator

$$
S_{\gamma}: u(r) \longmapsto e^{\left(\gamma-\frac{1}{2}\right) t} u\left(e^{-t}\right)
$$

and its inverse

$$
S_{\gamma}^{-1}: u(t) \longmapsto r^{\gamma-\frac{1}{2}} u(-\log r) .
$$

The operator (2.i) is well-defined as a topological isomorphism

$$
S_{\gamma}: \mathcal{D}^{\prime}\left(\mathbb{R}_{+}\right) \rightarrow \mathcal{D}^{\prime}(\mathbb{R})
$$

and restricts to topological isomorphisms on various subspaces, e. g.

$$
S_{\gamma}:\left\{\begin{array}{l}
C_{0}^{\infty}\left(\mathbb{R}_{+}\right) \longrightarrow C_{0}^{\infty}(\mathbb{R}) \\
\mathcal{T}_{\gamma}\left(\mathbb{R}_{+}\right) \longrightarrow \mathcal{S}(\mathbb{R})
\end{array}\right.
$$

This shows, in particular, that $\mathcal{T}_{\gamma}\left(\mathbb{R}_{+}\right)$is a nuclear Fréchet space with $C_{0}^{\infty}\left(\mathbb{R}_{+}\right)$as a dense subspace, and we have a canonical isomorphism $\mathcal{T}_{\gamma}\left(\mathbb{R}_{+}, E\right) \cong \mathcal{T}_{\gamma}\left(\mathbb{R}_{+}\right) \widehat{\otimes}_{\pi} E$.

- The (weighted) Mellin transform (defined on $C_{0}^{\infty}\left(\mathbb{R}_{+}\right)$)

$$
\left(\mathcal{M}_{\gamma} u\right)(z):=\int_{\mathbb{R}_{+}} r^{z} u(r) \frac{d r}{r}
$$

for $z \in \Gamma_{\frac{1}{2}-\gamma}$ with its inverse

$$
\left(\mathcal{M}_{\gamma}^{-1} u\right)(r):=\frac{1}{2 \pi i} \int_{\Gamma_{\frac{1}{2}-\gamma}} r^{-z} u(z) d z
$$

extends via $\mathcal{M}_{\gamma}=\mathcal{M}_{\gamma} \widehat{\otimes}_{\pi} \mathrm{id}_{E}$ to a topological isomorphism

$$
\mathcal{M}_{\gamma}: \mathcal{T}_{\gamma}\left(\mathbb{R}_{+}, E\right) \longrightarrow \mathcal{S}\left(\Gamma_{\frac{1}{2}-\gamma}, E\right) .
$$

For $u \in \mathcal{T}_{\gamma}\left(\mathbb{R}_{+}, E\right)$ we have

$$
\begin{gathered}
\mathcal{M}_{\gamma}\left(\left(-r \partial_{r}\right) u\right)(z)=z \mathcal{M}_{\gamma}(u)(z), \quad \mathcal{M}_{\gamma}((\log r) u)(z)=D_{\tau} \mathcal{M}_{\gamma}(u)(z), \\
\mathcal{M}_{\gamma+\delta}\left(r^{\delta} u\right)(z)=\mathcal{M}_{\gamma}(u)(z+\delta) .
\end{gathered}
$$


- For $u \in C_{0}^{\infty}\left(\mathbb{R}_{+}, E\right)$ the Mellin transform $\mathcal{M} u$ extends to an entire function such that $\left.\mathcal{M} u\right|_{\Gamma_{\frac{1}{2}-\gamma}}=\mathcal{M}_{\gamma} u$, and the mapping

$$
\mathbb{R} \ni \gamma \mapsto \mathcal{M}_{\gamma} u \in \mathcal{S}\left(\Gamma_{\frac{1}{2}-\gamma}, E\right)
$$

is a $C^{\infty}$-function taking values in the rapidly decreasing functions.

\subsection{A Paley-Wiener type theorem}

Let $E$ be a Fréchet space. For $r_{0} \in \mathbb{R}_{+}$and $\gamma \in \mathbb{R}$ let

$$
\mathcal{T}_{\gamma, 0}\left(\left(0, r_{0}\right), E\right):=\left\{u \in \mathcal{T}_{\gamma}\left(\mathbb{R}_{+}, E\right) ; \operatorname{supp}(u) \subseteq\left(0, r_{0}\right]\right\},
$$

and let

$$
\begin{aligned}
\mathcal{A}\left(\mathbb{H}_{\frac{1}{2}-\gamma}, E ; r_{0}\right):=\left\{f \in C^{\infty}\left(\mathbb{H}_{\frac{1}{2}-\gamma}, E\right) \cap \mathcal{A}\left(\stackrel{\circ}{\mathbb{H}}_{\frac{1}{2}-\gamma}, E\right)\right. & \\
{\left[\mathbb{H}_{\frac{1}{2}-\gamma} \ni z\right.} & \left.\left.\mapsto r_{0}^{-z} f(z) \in E\right] \in \mathcal{S}\left(\mathbb{H}_{\frac{1}{2}-\gamma}, E\right)\right\} .
\end{aligned}
$$

The latter is a Fréchet space with the projective topology with respect to the mapping

$$
\mathcal{A}\left(\mathbb{H}_{\frac{1}{2}-\gamma}, E ; r_{0}\right) \ni f \longmapsto r_{0}^{-z} f(z) \in \mathcal{S}\left(\mathbb{H}_{\frac{1}{2}-\gamma}, E\right) .
$$

Then the weighted Mellin transform $\mathcal{M}_{\gamma}: \mathcal{T}_{\gamma}\left(\mathbb{R}_{+}, E\right) \stackrel{\cong}{\longrightarrow} \mathcal{S}\left(\Gamma_{\frac{1}{2}-\gamma}, E\right)$ restricts to a topological isomorphism

$$
\mathcal{M}_{\gamma}: \mathcal{T}_{\gamma, 0}\left(\left(0, r_{0}\right), E\right) \stackrel{\cong}{\longrightarrow}\left(\mathbb{H}_{\frac{1}{2}-\gamma}, E ; r_{0}\right)
$$

for every $r_{0} \in \mathbb{R}_{+}$and every $\gamma \in \mathbb{R}$.

\subsection{The Mellin transform in distributions}

Let $E$ be a Hilbert space.

- For $\gamma \in \mathbb{R}$ the space $\mathcal{T}_{\gamma}^{\prime}\left(\mathbb{R}_{+}, E\right)$ consists of all continuous linear functionals $\mathcal{T}_{-\gamma}\left(\mathbb{R}_{+}\right) \rightarrow E$. Consequently, we have $\mathcal{T}_{\gamma}^{\prime}\left(\mathbb{R}_{+}, E\right) \subseteq \mathcal{D}^{\prime}\left(\mathbb{R}_{+}, E\right)$ in view of the density of $C_{0}^{\infty}\left(\mathbb{R}_{+}\right)$in $\mathcal{T}_{-\gamma}\left(\mathbb{R}_{+}\right)$.

- A distribution $u \in \mathcal{T}_{\gamma}^{\prime}\left(\mathbb{R}_{+}, E\right)$ is called regular if

$$
\langle u, \varphi\rangle=\int_{0}^{\infty} \tilde{u}(r) \varphi(r) d r, \quad \varphi \in \mathcal{T}_{-\gamma}\left(\mathbb{R}_{+}\right),
$$

for some Bochner measurable function $\tilde{u}$ such that $r^{-\left(\frac{1}{2}+\gamma\right)}\langle\log r\rangle^{-N} \tilde{u}(r) \in$ $L^{1}\left(\mathbb{R}_{+}, E\right)$ for some $N \in \mathbb{N}_{0}$. In particular, we have $\mathcal{T}_{\gamma}\left(\mathbb{R}_{+}, E\right) \subseteq \mathcal{T}_{\gamma}^{\prime}\left(\mathbb{R}_{+}, E\right)$, and more generally even $L^{2, \gamma}\left(\mathbb{R}_{+}, E\right):=r^{\gamma} L^{2}\left(\mathbb{R}_{+}, E\right) \subseteq \mathcal{T}_{\gamma}^{\prime}\left(\mathbb{R}_{+}, E\right)$ as regular distributions. 
- For every $\delta \in \mathbb{R}$ the product with functions $\psi \in C^{\infty}\left(\mathbb{R}_{+}\right)$such that $r^{-\delta}\left(-r \partial_{r}\right)^{\nu} \psi(r)$ is majorized by some power of $\langle\log r\rangle$, uniformly on $\mathbb{R}_{+}$for every $\nu \in \mathbb{N}_{0}$, provides an operator $\mathcal{T}_{\gamma}^{\prime}\left(\mathbb{R}_{+}, E\right) \rightarrow \mathcal{T}_{\gamma+\delta}^{\prime}\left(\mathbb{R}_{+}, E\right)$. Recall that $\langle\psi u, \varphi\rangle=\langle u, \psi \varphi\rangle$ for $\varphi \in \mathcal{T}_{-(\gamma+\delta)}\left(\mathbb{R}_{+}\right)$.

- The totally characteristic derivative $\left(-r \partial_{r}\right): \mathcal{D}^{\prime}\left(\mathbb{R}_{+}, E\right) \longrightarrow \mathcal{D}^{\prime}\left(\mathbb{R}_{+}, E\right)$ restricts to $\mathcal{T}_{\gamma}^{\prime}\left(\mathbb{R}_{+}, E\right)$, i. e. $\left(-r \partial_{r}\right)\left(\mathcal{T}_{\gamma}^{\prime}\left(\mathbb{R}_{+}, E\right)\right) \subseteq \mathcal{T}_{\gamma}^{\prime}\left(\mathbb{R}_{+}, E\right)$.

- The isomorphism $S_{\gamma}: \mathcal{D}^{\prime}\left(\mathbb{R}_{+}, E\right) \longrightarrow \mathcal{D}^{\prime}\left(\mathbb{R}_{+}, E\right)$ from (2.i) restricts to an isomorphism $S_{\gamma}: \mathcal{T}_{\gamma}^{\prime}\left(\mathbb{R}_{+}, E\right) \longrightarrow \mathcal{S}^{\prime}(\mathbb{R}, E)$. Note that we may write $\left\langle S_{\gamma} u, \varphi\right\rangle=\left\langle u, S_{-\gamma}^{-1} \varphi\right\rangle$ for $\varphi \in \mathcal{S}(\mathbb{R})$.

- The weighted Mellin transform $\mathcal{M}_{\gamma}$ extends to $\mathcal{T}_{\gamma}^{\prime}\left(\mathbb{R}_{+}, E\right)$ by means of the identity

$$
\left(\mathcal{M}_{\gamma} u\right)\left(\frac{1}{2}-\gamma+i \tau\right)=\left(\sqrt{2 \pi} \mathcal{F} S_{\gamma} u\right)(\tau)
$$

which provides an isomorphism $\mathcal{M}_{\gamma}: \mathcal{T}_{\gamma}^{\prime}\left(\mathbb{R}_{+}, E\right) \longrightarrow \mathcal{S}^{\prime}\left(\Gamma_{\frac{1}{2}-\gamma}, E\right)$. It restricts to an isomorphism

$$
\mathcal{M}_{\gamma}: L^{2, \gamma}\left(\mathbb{R}_{+}, E\right) \longrightarrow L^{2}\left(\Gamma_{\frac{1}{2}-\gamma}, E\right),
$$

and we have Parseval's identity

$$
\langle u, v\rangle_{L^{2, \gamma}\left(\mathbb{R}_{+}, E\right)}=\frac{1}{2 \pi}\left\langle\mathcal{M}_{\gamma} u, \mathcal{M}_{\gamma} v\right\rangle_{L^{2}\left(\Gamma_{\frac{1}{2}-\gamma}, E\right)} .
$$

The relations in (2.iii) hold in the distributional sense.

\section{The calculus of Volterra symbols}

\subsection{General anisotropic parameter-dependent symbols}

3.1 Definition. Let $\ell \in \mathbb{N}$ be a given anisotropy.

a) For $(\xi, \lambda) \in \mathbb{R}^{n} \times \mathbb{R}^{q}$ define

$$
\begin{aligned}
|\xi, \lambda|_{\ell} & :=\left(|\xi|^{2 \ell}+|\lambda|^{2}\right)^{\frac{1}{2 \ell}} \\
\langle\xi, \lambda\rangle_{\ell} & :=\left(1+|\xi|^{2 \ell}+|\lambda|^{2}\right)^{\frac{1}{2 \ell}},
\end{aligned}
$$

where $|\cdot|$ denotes the Euclidean norm.

b) For a multi-index $\beta=\left(\alpha, \alpha^{\prime}\right) \in \mathbb{N}_{0}^{n+q}$ let

$$
|\beta|_{\ell}:=|\alpha|+\ell \cdot\left|\alpha^{\prime}\right|,
$$

where $|\cdot|$ denotes the usual length of a multi-index as the sum of its components. 
3.2 Lemma. There exists a constant $c>0$ such that for all $s \in \mathbb{R}$ and $\xi_{1}, \xi_{2} \in$ $\mathbb{R}^{n}, \lambda_{1}, \lambda_{2} \in \mathbb{R}^{q}$ the following inequality is fulfilled (Peetre's inequality):

$$
\left\langle\xi_{1}+\xi_{2}, \lambda_{1}+\lambda_{2}\right\rangle_{\ell}^{s} \leq c^{|s|}\left\langle\xi_{1}, \lambda_{1}\right\rangle_{\ell}^{|s|}\left\langle\xi_{2}, \lambda_{2}\right\rangle_{\ell}^{s} .
$$

Morover, we can compare the regularized "anisotropic distance" $\langle\cdot, \cdot\rangle_{\ell}$ with the "isotropic distance", i. e., there exist constants $c_{1}, c_{2}>0$ such that

$$
c_{1}\langle\xi, \lambda\rangle_{\ell} \leq\langle\xi, \lambda\rangle \leq c_{2}\langle\xi, \lambda\rangle_{\ell}^{\ell} .
$$

3.3 Definition. Let $E$ be a Hilbert space. A strongly continuous group-action on $E$ is a strongly continuous group-representation

$$
\kappa:\left(\mathbb{R}_{+}, \cdot\right) \longrightarrow \mathcal{L}(E) .
$$

From the uniform boundedness principle we obtain the existence of constants $c, M \geq 0$ such that

$$
\left\|\kappa_{\varrho}\right\|_{\mathcal{L}(E)} \leq c \max \left\{\varrho, \frac{1}{\varrho}\right\}^{M} \text { for } \varrho \in \mathbb{R}_{+} .
$$

By the trivial group-action we mean the trivial representation, i. e., $\kappa_{\varrho}=\operatorname{Id}_{E}$ for all $\varrho \in \mathbb{R}_{+}$.

3.4 Definition. Let $E$ and $\tilde{E}$ be Hilbert spaces endowed with strongly continuous group-actions $\left\{\kappa_{\varrho}\right\}$ and $\left\{\tilde{\kappa}_{\varrho}\right\}$, respectively. For $\mu \in \mathbb{R}$ we define

$$
\begin{aligned}
& S^{\mu ; \ell}\left(\mathbb{R}^{n} \times \mathbb{R}^{q} ; E, \tilde{E}\right):=\left\{a \in C^{\infty}\left(\mathbb{R}^{n} \times \mathbb{R}^{q}, \mathcal{L}(E, \tilde{E})\right) ; \text { for all } k \in \mathbb{N}_{0}:\right. \\
& \left.p_{k}(a):=\sup _{\substack{(\xi, \lambda) \in \mathbb{R}^{n} \times \mathbb{R}^{q} \\
|\beta|_{\ell} \leq k}}\left\|\tilde{\kappa}_{\langle\xi, \lambda\rangle_{\ell}}^{-1} \partial_{(\xi, \lambda)}^{\beta} a(\xi, \lambda) \kappa_{\langle\xi, \lambda\rangle_{\ell}}\right\|\langle\xi, \lambda\rangle_{\ell}^{-\mu+|\beta|_{\ell}}<\infty\right\} .
\end{aligned}
$$

This is a Fréchet space with the topology induced by the seminorm-system $\left\{p_{k} ; k \in\right.$ $\left.\mathbb{N}_{0}\right\}$. Define

$$
S^{-\infty}\left(\mathbb{R}^{n} \times \mathbb{R}^{q} ; E, \tilde{E}\right):=\bigcap_{\mu \in \mathbb{R}} S^{\mu ; \ell}\left(\mathbb{R}^{n} \times \mathbb{R}^{q} ; E, \tilde{E}\right) .
$$

By (3.ii) and Definition 3.3 this space does not depend on $\ell \in \mathbb{N}$ and the groupactions involved on $E$ and $\tilde{E}$, and we have $S^{-\infty}\left(\mathbb{R}^{n} \times \mathbb{R}^{q} ; E, \tilde{E}\right)=\mathcal{S}\left(\mathbb{R}^{n} \times\right.$ $\left.\mathbb{R}^{q}, \mathcal{L}(E, \tilde{E})\right)$. If $E=\tilde{E}=\mathbb{C}$ with the trivial group-action involved we suppress the Hilbert spaces from the notation.

More generally, let $\left\{E_{j}\right\}_{j \in \mathbb{N}}$ and $\left\{\tilde{E}_{j}\right\}_{j \in \mathbb{N}}$ be scales of Hilbert spaces such that $E_{j} \hookrightarrow E_{j+1}$ and $\tilde{E}_{j+1} \hookrightarrow \tilde{E}_{j}$ for $j \in \mathbb{N}$. Moreover, let $\left\{\kappa_{\varrho}\right\}$ and $\left\{\tilde{\kappa}_{\varrho}\right\}$ be defined on the unions of the $\left\{E_{j}\right\}$ and $\left\{\tilde{E}_{j}\right\}$, respectively, such that the restrictions on each $E_{j}$ and $\tilde{E}_{j}$ are strongly continuous group-actions. Define

$$
S^{\mu ; \ell}\left(\mathbb{R}^{n} \times \mathbb{R}^{q} ; \underset{j \in \mathbb{N}}{\operatorname{ind}-\lim } E_{j}, \underset{k \in \mathbb{N}}{\operatorname{proj-lim}} \tilde{E}_{k}\right):=\bigcap_{j, k \in \mathbb{N}} S^{\mu ; \ell}\left(\mathbb{R}^{n} \times \mathbb{R}^{q} ; E_{j}, \tilde{E}_{k}\right)
$$


with the natural Fréchet topologies induced. The spaces of order $-\infty$ are defined in an analogous manner. With this notion the case of single Hilbert spaces $E$ and $\tilde{E}$ corresponds to the constant scales.

3.5 Notation. Let $\left\{E_{j}\right\}$ and $\left\{\tilde{E}_{j}\right\}$ be scales of Hilbert spaces with group-actions $\left\{\kappa_{\varrho}\right\}$ and $\left\{\tilde{\kappa}_{\varrho}\right\}$, respectively, in the sense of Definition 3.4. For short, we set

$$
\mathcal{E}:=\underset{j \in \mathbb{N}}{\operatorname{ind}-\lim } E_{j} \quad \text { and } \quad \tilde{\mathcal{E}}:=\underset{j \in \mathbb{N}}{\operatorname{proj}-\lim } \tilde{E}_{j}
$$

3.6 Lemma. Let $E, \tilde{E}$ and $\hat{E}$ be Hilbert-spaces with strongly continuous groupactions $\left\{\kappa_{\varrho}\right\},\left\{\tilde{\kappa}_{\varrho}\right\}$, and $\left\{\hat{\kappa}_{\varrho}\right\}$.

a) For $\mu \geq \mu^{\prime}$ the embedding $S^{\mu^{\prime} ; \ell}\left(\mathbb{R}^{n} \times \mathbb{R}^{q} ; E, \tilde{E}\right) \hookrightarrow S^{\mu ; \ell}\left(\mathbb{R}^{n} \times \mathbb{R}^{q} ; E, \tilde{E}\right)$ is well-defined and continuous.

b) The embeddings $S^{\mu ; \ell}\left(\mathbb{R}^{n} \times \mathbb{R}^{q} ; E, \tilde{E}\right) \hookrightarrow S_{\perp}^{\mu+M+\tilde{M} ; \ell}\left(\mathbb{R}^{n} \times \mathbb{R}^{q} ; E, \tilde{E}\right)$ and $S_{1}^{\mu ; \ell}\left(\mathbb{R}^{n} \times \mathbb{R}^{q} ; E, \tilde{E}\right) \hookrightarrow S^{\mu+M+\tilde{M} ; \ell}\left(\mathbb{R}^{n} \times \mathbb{R}^{q} ; E, \tilde{E}\right)$ are well-defined and continuous, where the subscript 1 indicates that the trivial group-actions are involved on the spaces $E$ and $\tilde{E}$. Here $M$ and $\tilde{M}$ are the constants in the estimates for the operator-norms of the group-actions from (3.iv).

c) For $\beta \in \mathbb{N}_{0}^{n+q}$ the operator of differentiation $\partial_{(\xi, \lambda)}^{\beta}: S^{\mu ; \ell}\left(\mathbb{R}^{n} \times \mathbb{R}^{q} ; E, \tilde{E}\right) \rightarrow$ $S^{\mu-|\beta|_{\ell} ; \ell}\left(\mathbb{R}^{n} \times \mathbb{R}^{q} ; E, \tilde{E}\right)$ is continuous.

d) For $\mu, \mu^{\prime} \in \mathbb{R}$ pointwise multiplication (composition of operators) induces a continuous bilinear mapping

$$
S^{\mu ; \ell}\left(\mathbb{R}^{n} \times \mathbb{R}^{q} ; \tilde{E}, \hat{E}\right) \times S^{\mu ; \ell}\left(\mathbb{R}^{n} \times \mathbb{R}^{q} ; E, \tilde{E}\right) \rightarrow S^{\mu+\mu^{\prime} ; \ell}\left(\mathbb{R}^{n} \times \mathbb{R}^{q} ; E, \hat{E}\right) .
$$

\subsubsection{Asymptotic expansion}

3.7 Definition. Let $\mathcal{E}$ and $\tilde{\mathcal{E}}$ be associated to scales of Hilbert spaces according to Notation 3.5.

Let $\left(\mu_{k}\right) \subseteq \mathbb{R}$ be a sequence of reals such that $\mu_{k} \underset{k \rightarrow \infty}{\longrightarrow}-\infty$ and $\bar{\mu}:=\max _{k \in \mathbb{N}} \mu_{k}$. Moreover, let $a_{k} \in S^{\mu_{k} ; \ell}\left(\mathbb{R}^{n} \times \mathbb{R}^{q} ; \mathcal{E}, \tilde{\mathcal{E}}\right)$. A symbol $a \in S^{\bar{\mu} ; \ell}\left(\mathbb{R}^{n} \times \mathbb{R}^{q} ; \mathcal{E}, \tilde{\mathcal{E}}\right)$ is called the asymptotic expansion of the $a_{k}$, if for every $R \in \mathbb{R}$ there is a $k_{0} \in \mathbb{N}$ such that for $k>k_{0}$

$$
a-\sum_{j=1}^{k} a_{j} \in S^{R ; \ell}\left(\mathbb{R}^{n} \times \mathbb{R}^{q} ; \mathcal{E}, \tilde{\mathcal{E}}\right) .
$$

The symbol $a$ is uniquely determined modulo $S^{-\infty}\left(\mathbb{R}^{n} \times \mathbb{R}^{q} ; \mathcal{E}, \tilde{\mathcal{E}}\right)$.

For short, we write $a \sim \sum_{j=1}^{\infty} a_{j}$. 
3.8 Remark. Given a sequence of symbols $a_{j}$ according to Definition 3.7, there exists a symbol $a$ such that $a \sim \sum_{j=1}^{\infty} a_{j}$. The proof relies on a Borel argument, and it can be found in the standard monographs about pseudodifferential operators (see, e. g., Shubin [48]). More precisely, the symbol $a$ is given as a convergent series

$$
a(\xi, \lambda):=\sum_{j=1}^{\infty} \chi\left(\frac{\xi}{c_{j}}, \frac{\lambda}{c_{j}^{\ell}}\right) a_{j}(\xi, \lambda)
$$

where $\chi \in C^{\infty}\left(\mathbb{R}^{n} \times \mathbb{R}^{q}\right)$ is a 0 -excision function, i. e., $\chi \equiv 0$ near 0 and $\chi \equiv 1$ near infinity, and $\left(c_{j}\right) \subseteq \mathbb{R}_{+}$such that $c_{j} \rightarrow \infty$ as $j \rightarrow \infty$ sufficiently fast.

This argument extends to a variety of more general symbol classes, in particular, when an additional dependence of variables is involved. However, it breaks down for holomorphic symbols, for the analyticity in the covariables is not preserved.

\section{$3.2 \quad$ Homogeneity and classical symbols}

3.9 Definition. Let $E$ and $\tilde{E}$ be Hilbert spaces with group-actions $\left\{\kappa_{\varrho}\right\}$ and $\left\{\tilde{\kappa}_{\varrho}\right\}$, respectively. A function $f:\left(\mathbb{R}^{n} \times \mathbb{R}^{q}\right) \backslash\{0\} \rightarrow \mathcal{L}(E, \tilde{E})$ is called (anisotropic) homogeneous of degree $\mu \in \mathbb{R}$, if for $(\xi, \lambda) \in\left(\mathbb{R}^{n} \times \mathbb{R}^{q}\right) \backslash\{0\}$ and $\varrho>0$

$$
f\left(\varrho \xi, \varrho^{\ell} \lambda\right)=\varrho^{\mu} \tilde{\kappa}_{\varrho} f(\xi, \lambda) \kappa_{\varrho}^{-1} .
$$

A function $f: \mathbb{R}^{n} \times \mathbb{R}^{q} \rightarrow \mathcal{L}(E, \tilde{E})$ is called (anisotropic) homogeneous of degree $\mu \in \mathbb{R}$ for large $(\xi, \lambda)$, if for $(\xi, \lambda) \in \mathbb{R}^{n} \times \mathbb{R}^{q}$ with $|(\xi, \lambda)|$ sufficiently large and $\varrho \geq 1$

$$
f\left(\varrho \xi, \varrho^{\ell} \lambda\right)=\varrho^{\mu} \tilde{\kappa}_{\varrho} f(\xi, \lambda) \kappa_{\varrho}^{-1} .
$$

In this work, homogeneity always is meant in this anisotropic sense.

3.10 Lemma. Let $a \in C^{\infty}\left(\mathbb{R}^{n} \times \mathbb{R}^{q}, \mathcal{L}(E, \tilde{E})\right)$ be homogeneous of degree $\mu \in \mathbb{R}$ for large $(\xi, \lambda)$. Then $a \in S^{\mu ; \ell}\left(\mathbb{R}^{n} \times \mathbb{R}^{q} ; E, \tilde{E}\right)$.

Proof. By differentiating relation (3.vi) we see, that for $\beta \in \mathbb{N}_{0}^{n+q}$ the function $\partial_{(\xi, \lambda)}^{\beta} a$ is homogeneous of degree $\mu-|\beta|_{\ell}$ for large $(\xi, \lambda)$. Thus it suffices to show that $\left\|\tilde{\kappa}_{\langle\xi, \lambda\rangle_{\ell}}^{-1} a(\xi, \lambda) \kappa_{\langle\xi, \lambda\rangle_{\ell}}\right\|=O\left(\langle\xi, \lambda\rangle_{\ell}^{\mu}\right)$ for $|(\xi, \lambda)| \rightarrow \infty$. Extension by homogeneity shows that there is a homogeneous function $f$ of degree $\mu$ such that $a=f$ for large $|(\xi, \lambda)|$. Note that $f$ is continuous on $\left(\mathbb{R}^{n} \times \mathbb{R}^{q}\right) \backslash\{0\}$ with values in $\mathcal{L}_{\sigma}(E, \tilde{E})$ for the group-actions are strongly continuous. In particular, $f$ maps compact sets to bounded sets in $\mathcal{L}(E, \tilde{E})$ by the uniform boundedness principle. Employing the 
identity

$$
\begin{aligned}
\tilde{\kappa}_{\langle\xi, \lambda\rangle_{\ell}}^{-1} f(\xi, \lambda) \kappa_{\langle\xi, \lambda\rangle_{\ell}} & =\tilde{\kappa}_{\langle\xi, \lambda\rangle_{\ell}}^{-1} f\left(|\xi, \lambda|_{\ell} \frac{\xi}{|\xi, \lambda|_{\ell}},|\xi, \lambda|_{\ell}^{\ell} \frac{\lambda}{|\xi, \lambda|_{\ell}^{\ell}}\right) \kappa_{\langle\xi, \lambda\rangle_{\ell}} \\
& =|\xi, \lambda|_{\ell}^{\mu} \tilde{\kappa}_{\langle\xi, \lambda\rangle_{\ell}^{-1}|\xi, \lambda|_{\ell}} f\left(\frac{\xi}{|\xi, \lambda|_{\ell}}, \frac{\lambda}{|\xi, \lambda|_{\ell}^{\ell}}\right) \kappa_{|\xi, \lambda|_{\ell}^{-1}\langle\xi, \lambda\rangle_{\ell}}
\end{aligned}
$$

for $(\xi, \lambda) \neq 0$ yields that $\left\|\tilde{\kappa}_{\langle\xi, \lambda\rangle_{\ell}}^{-1} f(\xi, \lambda) \kappa_{\langle\xi, \lambda\rangle_{\ell}}\right\|=O\left(\langle\xi, \lambda\rangle_{\ell}^{\mu}\right)$ for $|(\xi, \lambda)| \rightarrow \infty$. This proves the lemma.

3.11 Corollary. For $\mu \in \mathbb{R}$ the function $\langle\cdot, \cdot\rangle_{\ell}^{\mu}$ belongs to $S^{\mu ; \ell}\left(\mathbb{R}^{n} \times \mathbb{R}^{q}\right)$.

Proof. Induction over the length $|\beta|$ of $\beta \in \mathbb{N}_{0}^{n+q}$ shows

$$
\partial_{(\xi, \lambda)}^{\beta}\langle\xi, \lambda\rangle_{\ell}^{\mu}=\sum_{k=0}^{|\beta|} p_{\beta, k}(\xi, \lambda)\langle\xi, \lambda\rangle_{\ell}^{\mu-2 k l}
$$

with suitable polynomials $p_{\beta, k}$ that are (anisotropic) homogeneous of degree $2 k l-$ $|\beta|_{\ell}$. From Lemma 3.10 we obtain the assertion.

3.12 Definition. Let $E$ and $\tilde{E}$ be Hilbert spaces with group-actions $\left\{\kappa_{\varrho}\right\}$ and $\left\{\tilde{\kappa}_{\varrho}\right\}$, respectively. Let $\mu \in \mathbb{R}$. We define

$$
S_{c l}^{\mu ; \ell}\left(\mathbb{R}^{n} \times \mathbb{R}^{q} ; E, \tilde{E}\right):=\left\{a \in S^{\mu ; \ell}\left(\mathbb{R}^{n} \times \mathbb{R}^{q} ; E, \tilde{E}\right) ; a \sim \sum_{k=0}^{\infty} \chi a_{(\mu-k)}\right\}
$$

where $\chi \in C^{\infty}\left(\mathbb{R}^{n} \times \mathbb{R}^{q}\right)$ is a 0-excision function and $a_{(\mu-k)} \in C^{\infty}\left(\left(\mathbb{R}^{n} \times \mathbb{R}^{q}\right) \backslash\right.$ $\{0\}, \mathcal{L}(E, \tilde{E}))$ are (anisotropic) homogeneous functions of degree $\mu-k$, the so called homogeneous components of $a$.

3.13 Remark. By Lemma 3.10 the space $S_{c l}^{\mu ; \ell}\left(\mathbb{R}^{n} \times \mathbb{R}^{q} ; E, \tilde{E}\right)$ is well-defined.

The homogeneous components of $a \in S_{c l}^{\mu ; \ell}\left(\mathbb{R}^{n} \times \mathbb{R}^{q} ; E, \tilde{E}\right)$ are uniquely determined by $a$. They can iteratively be recovered from the relation

$$
\frac{1}{\varrho^{\mu-k}} \tilde{\kappa}_{\varrho}^{-1}\left(a\left(\varrho \xi, \varrho^{\ell} \lambda\right)-\sum_{j=0}^{k-1} a_{(\mu-j)}\left(\varrho \xi, \varrho^{\ell} \lambda\right)\right) \kappa_{\varrho} \underset{\varrho \rightarrow \infty}{\longrightarrow} a_{(\mu-k)}(\xi, \lambda)
$$

with convergence in $\mathcal{L}(E, \tilde{E})$, which holds locally uniformly for $0 \neq(\xi, \lambda) \in \mathbb{R}^{n} \times$ $\mathbb{R}^{q}$.

Note that $S_{c l}^{\mu ; \ell}\left(\mathbb{R}^{n} \times \mathbb{R}^{q} ; E, \tilde{E}\right)$ is a Fréchet space with respect to the projective topology of the mappings

$$
S_{c l}^{\mu ; \ell}\left(\mathbb{R}^{n} \times \mathbb{R}^{q} ; E, \tilde{E}\right) \ni a \longmapsto\left\{\begin{aligned}
a-\sum_{j=0}^{k-1} \chi a_{(\mu-j)} & \in S^{\mu-k ; \ell}\left(\mathbb{R}^{n} \times \mathbb{R}^{q} ; E, \tilde{E}\right) \\
a_{(\mu-k)} & \in C^{\infty}\left(\left(\mathbb{R}^{n} \times \mathbb{R}^{q}\right) \backslash\{0\}, \mathcal{L}(E, \tilde{E})\right)
\end{aligned}\right.
$$


for $k \in \mathbb{N}_{0}$.

The space of classical symbols is closed with respect to taking asymptotic expansions. More precisely, let $\mu \in \mathbb{R}$ and $a^{\mu-j} \in S_{c l}^{\mu-j ; \ell}\left(\mathbb{R}^{n} \times \mathbb{R}^{q} ; E, \tilde{E}\right)$ for $j \in \mathbb{N}_{0}$. Then there is a symbol $a \in S_{c l}^{\mu ; \ell}\left(\mathbb{R}^{n} \times \mathbb{R}^{q} ; E, \tilde{E}\right)$ such that $a \sim \sum_{j=0}^{\infty} a^{\mu-j}$. In fact, the homogeneous components of $a$ are given by

$$
a_{(\mu-k)}=\sum_{j \leq k} a_{(\mu-k)}^{\mu-j}
$$

for $k \in \mathbb{N}_{0}$.

Analogously, we define the spaces of classical symbols when we start from scales of Hilbert spaces $\left\{E_{j}\right\}$ and $\left\{\tilde{E}_{j}\right\}$ instead of single spaces.

Proof. Because of its importance we prove relation (3.vii):

Note first, that for $(\xi, \lambda) \in K \Subset\left(\mathbb{R}^{n} \times \mathbb{R}^{q}\right) \backslash\{0\}$ we have $\chi\left(\varrho \xi, \varrho^{\ell} \lambda\right) \equiv 1$ for sufficiently large $\varrho$ since $\chi$ is a 0 -excision function. Therefore, we see for $\varrho$ sufficiently large on $K$ :

$$
\begin{aligned}
\left\|\frac{1}{\varrho^{\mu-k}} \tilde{\kappa}_{\varrho}^{-1}\left(a\left(\varrho \xi, \varrho^{\ell} \lambda\right)-\sum_{j=0}^{k-1} a_{(\mu-j)}\left(\varrho \xi, \varrho^{\ell} \lambda\right)\right) \kappa_{\varrho}-a_{(\mu-k)}(\xi, \lambda)\right\| \\
\quad=\left\|\frac{1}{\varrho^{\mu-k}} \tilde{\kappa}_{\varrho}^{-1}\left(a\left(\varrho \xi, \varrho^{\ell} \lambda\right)-\sum_{j=0}^{k} \chi\left(\varrho \xi, \varrho^{\ell} \lambda\right) a_{(\mu-j)}\left(\varrho \xi, \varrho^{\ell} \lambda\right)\right) \kappa_{\varrho}\right\| \\
\leq \text { Const } \cdot\left\|\tilde{\kappa}_{\varrho^{-1}}\left\langle\varrho \xi, \varrho^{\ell} \lambda\right\rangle_{\ell}\right\| \cdot\left\|\kappa_{\left\langle\varrho \xi, \varrho^{\ell} \lambda\right\rangle_{\ell}^{-1} \varrho}\right\| \cdot \frac{1}{\varrho^{\mu-k}}\left\langle\varrho \xi, \varrho^{\ell} \lambda\right\rangle_{\ell}^{\mu-k-1} \\
\leq \text { Const } \cdot \frac{1}{\varrho^{\mu-k}}\left\langle\varrho \xi, \varrho^{\ell} \lambda\right\rangle_{\ell}^{\mu-k-1} \underset{\varrho \rightarrow \infty}{\longrightarrow} 0
\end{aligned}
$$

uniformly for $(\xi, \lambda) \in K$.

3.14 Remark. The considerations about general anisotropic symbols carry over to the case, where the space $\mathbb{R}^{q}$ is replaced by a conical subset $\emptyset \neq \Lambda \subseteq \mathbb{R}^{q}$, which is the closure of its interior. There only arise notational modifications. In this work, we will mainly make use of symbols and operators with either $\Lambda=\mathbb{R}$ (more precisely, a vertical line $\Lambda=\Gamma_{\beta}$ in $\mathbb{C}$ parallel to the imaginary axis), or with the right half-plane $\Lambda=\mathbb{H}_{0} \subseteq \mathbb{C} \cong \mathbb{R}^{2}$.

\subsection{Parameter-dependent Volterra symbols}

Let

$$
\mathbb{H}_{0}:=\{z \in \mathbb{C} ; \operatorname{Re}(z) \geq 0\} \subseteq \mathbb{C} \cong \mathbb{R}^{2}
$$


be the right half-plane in $\mathbb{C}$. The significant property of Volterra symbols is, that in addition to the symbol estimates we employ the analyticity in the interior of $\mathbb{H}_{0}$.

3.15 Definition. Let $E$ and $\tilde{E}$ be Hilbert spaces endowed with strongly continuous group-actions $\left\{\kappa_{\varrho}\right\}$ and $\left\{\tilde{\kappa}_{\varrho}\right\}$, respectively. For $\mu \in \mathbb{R}$ we define

$$
S_{V(c l)}^{\mu ; \ell}\left(\mathbb{R}^{n} \times \mathbb{H}_{0} ; E, \tilde{E}\right):=S_{(c l)}^{\mu ; \ell}\left(\mathbb{R}^{n} \times \mathbb{H}_{0} ; E, \tilde{E}\right) \cap \mathcal{A}\left(\stackrel{\circ}{\mathbb{H}_{0}}, C^{\infty}\left(\mathbb{R}^{n}, \mathcal{L}(E, \tilde{E})\right)\right)
$$

which is a closed subspace of $S_{(c l)}^{\mu ; \ell}\left(\mathbb{R}^{n} \times \mathbb{H}_{0} ; E, \tilde{E}\right)$. Analogously, we define

$$
S_{V}^{-\infty}\left(\mathbb{R}^{n} \times \mathbb{H}_{0} ; E, \tilde{E}\right):=\bigcap_{\mu \in \mathbb{R}} S_{V}^{\mu ; \ell}\left(\mathbb{R}^{n} \times \mathbb{H}_{0} ; E, \tilde{E}\right)
$$

These symbols are called symbols with the Volterra property, or simply Volterra symbols, which is indicated by the subscript $V$. Of course, this notion also applies to the case of scales of Hilbert spaces involved instead of single spaces, and we shall employ the same conventions as in the case without the extra analyticity condition, see Definition 3.4.

From the definition we obtain that the properties in Lemma 3.6 apply to symbols with the Volterra property, i. e., the analyticity condition remains preserved.

3.16 Proposition. a) The restriction of the parameter to the imaginary axis induces a continuous embedding $S_{V(c l)}^{\mu ; \ell}\left(\mathbb{R}^{n} \times \mathbb{H}_{0} ; E, \tilde{E}\right) \hookrightarrow S_{(c l)}^{\mu ; \ell}\left(\mathbb{R}^{n} \times \Gamma_{0} ; E, \tilde{E}\right)$. Notice that we identify $\Gamma_{0} \cong \mathbb{R}$.

b) The homogeneous components of a symbol $a \in S_{V c l}^{\mu ; \ell}\left(\mathbb{R}^{n} \times \mathbb{H}_{0} ; E, \tilde{E}\right)$ are analytic in the interior of $\mathbb{H}_{0}$.

Proof. The first assertion is immediate. Note that the homogeneous components of the "restricted" symbol in the classical case originate from the restrictions of the homogeneous components to the real line. The second assertion follows by induction from relation (3.vii) in Remark 3.13 together with the Weierstrass approximation theorem.

3.17 Definition. Let $\mathcal{E}$ and $\tilde{\mathcal{E}}$ be scales of Hilbert spaces with group-actions $\left\{\kappa_{\varrho}\right\}$ and $\left\{\tilde{\kappa}_{\varrho}\right\}$, respectively, as in Notation 3.5. Let $\left(\mu_{k}\right) \subseteq \mathbb{R}$ be a sequence of reals such that $\mu_{k} \underset{k \rightarrow \infty}{\longrightarrow}-\infty$, and $\bar{\mu}:=\max _{k \in \mathbb{N}} \mu_{k}$. Moreover, let $a_{k} \in S_{V}^{\mu_{k} ; \ell}\left(\mathbb{R}^{n} \times \mathbb{H}_{0} ; \mathcal{E}, \tilde{\mathcal{E}}\right)$. A symbol $a \in S_{V}^{\bar{\mu} ; \ell}\left(\mathbb{R}^{n} \times \mathbb{H}_{0} ; \mathcal{E}, \tilde{\mathcal{E}}\right)$ is called the asymptotic expansion of the $a_{k}$, if for every $R \in \mathbb{R}$ there is a $k_{0} \in \mathbb{N}$ such that for $k>k_{0}$

$$
a-\sum_{j=1}^{k} a_{j} \in S_{V}^{R ; \ell}\left(\mathbb{R}^{n} \times \mathbb{H}_{0} ; \mathcal{E}, \tilde{\mathcal{E}}\right) .
$$


The symbol $a$ is uniquely determined modulo $S_{V}^{-\infty}\left(\mathbb{R}^{n} \times \mathbb{H}_{0} ; \mathcal{E}, \tilde{\mathcal{E}}\right)$.

For short, we write $a \underset{V}{\sim} \sum_{j=1}^{\infty} a_{j}$.

3.18 Remark. Note that the notion of asymptotic expansion for Volterra symbols from Definition 3.17 is more refined than that of Definition 3.7. We distinguish here between the notions of asymptotic expansions for symbols with and without the Volterra property, i. e., for symbols with and without the extra analyticity condition in the half-plane.

In particular, the proof of the existence of a Volterra symbol having a prescribed asymptotic expansion is more complicated than in the standard setting of general symbols (see Remark 3.8), but the result holds true also in this case. A suitable way to achieve it makes use of a Borel argument, where kernel cut-off techniques are used to replace the excision function in the covariables, such that the analyticity in the half-plane remains preserved; see, e. g., Krainer [23], [24], and Section 5.1 — especially Remark 5.4 - below.

\subsubsection{The translation operator in Volterra symbols}

3.19 Definition. Let $\mathcal{E}$ and $\tilde{\mathcal{E}}$ be scales of Hilbert spaces according to Notation 3.5. For $\tau \geq 0$ define the translation operator $T_{\tau}$ on $S_{V}^{\mu ; \ell}\left(\mathbb{R}^{n} \times \mathbb{H}_{0} ; \mathcal{E}, \tilde{\mathcal{E}}\right)$ via

$$
\left(T_{\tau} a\right)(\xi, \lambda):=a(\xi, \lambda+\tau) .
$$

3.20 Proposition. For every $\tau \geq 0$ the translation operator $T_{\tau}$ acts linear and continuous in the spaces

$$
T_{\tau}: S_{V(c l)}^{\mu ; \ell}\left(\mathbb{R}^{n} \times \mathbb{H}_{0} ; \mathcal{E}, \tilde{\mathcal{E}}\right) \longrightarrow S_{V(c l)}^{\mu ; \ell}\left(\mathbb{R}^{n} \times \mathbb{H}_{0} ; \mathcal{E}, \tilde{\mathcal{E}}\right) .
$$

Moreover, $T_{\tau} a$ has the following asymptotic expansion in terms of $\tau$ and $a$ :

$$
T_{\tau} a \underset{V}{\sim} \sum_{k=0}^{\infty} \frac{\tau^{k}}{k !} \cdot \partial_{\lambda}^{k} a .
$$

In particular, the operator $I-T_{\tau}$ is continuous in the spaces

$$
I-T_{\tau}: S_{V(c l)}^{\mu ; \ell}\left(\mathbb{R}^{n} \times \mathbb{H}_{0} ; \mathcal{E}, \tilde{\mathcal{E}}\right) \longrightarrow S_{V(c l)}^{\mu-\ell ; \ell}\left(\mathbb{R}^{n} \times \mathbb{H}_{0} ; \mathcal{E}, \tilde{\mathcal{E}}\right) .
$$

Proof. Without loss of generality we may restrict to single Hilbert spaces $E$ and $\tilde{E}$. For $T_{\tau}$ acts continuously in $\mathcal{A}\left(\mathbb{H}_{0}, C^{\infty}\left(\mathbb{R}^{n}, \mathcal{L}(E, \tilde{E})\right)\right) \cap C^{\infty}\left(\mathbb{R}^{n} \times \mathbb{H}_{0}, \mathcal{L}(E, \tilde{E})\right)$ we only have to check that $T_{\tau} a \in S_{V}^{\mu ; \ell}\left(\mathbb{R}^{n} \times \mathbb{H}_{0} ; E, \tilde{E}\right)$ for $a \in S_{V}^{\mu ; \ell}\left(\mathbb{R}^{n} \times \mathbb{H}_{0} ; E, \tilde{E}\right)$, which is trivially fulfilled in view of (3.i) and (3.iv), as well as the asymptotic 
expansion of $T_{\tau} a$ in terms of $\tau$ and $a$. Then the closed graph theorem implies the remaining assertions.

Carrying out a Taylor expansion in $\tau=0$ implies that for each $N \in \mathbb{N}$ we may write

$$
a(\xi, \lambda+\tau)=\sum_{k=0}^{N-1} \frac{\tau^{k}}{k !} \cdot \partial_{\lambda}^{k} a(\xi, \lambda)+\frac{\tau^{N}}{(N-1) !} \int_{0}^{1}(1-\theta)^{N-1}\left(\partial_{\lambda}^{N} a\right)(\xi, \lambda+\theta \tau) d \theta
$$

For the integrand in the remainder may be regarded as a continuous function in $\theta \in[0,1]$ with values in $S_{V}^{\mu-N \ell ; \ell}\left(\mathbb{R}^{n} \times \mathbb{H}_{0} ; E, \tilde{E}\right)$, we obtain the desired asymptotic expansion for $T_{\tau} a$.

3.21 Notation. Let $E$ and $\tilde{E}$ be Hilbert spaces endowed with group-actions as before. For $\mu \in \mathbb{R}$ let $S^{(\mu ; \ell)}\left(\left(\mathbb{R}^{n} \times \mathbb{H}_{0}\right) \backslash\{0\} ; E, \tilde{E}\right)$ denote the closed subspace of $C^{\infty}\left(\left(\mathbb{R}^{n} \times \mathbb{H}_{0}\right) \backslash\{0\}, \mathcal{L}(E, \tilde{E})\right)$ consisting of all anisotropic homogeneous functions of degree $\mu$. Moreover, let

$$
\begin{aligned}
S_{V}^{(\mu ; \ell)}\left(\left(\mathbb{R}^{n} \times \mathbb{H}_{0}\right) \backslash\{0\} ; E, \tilde{E}\right):=S^{(\mu ; \ell)}\left(\left(\mathbb{R}^{n} \times \mathbb{H}_{0}\right) \backslash\{0\} ; E, \tilde{E}\right) \\
\\
\cap \mathcal{A}\left(\mathbb{H}_{0}, C^{\infty}\left(\mathbb{R}^{n}, \mathcal{L}(E, \tilde{E})\right)\right),
\end{aligned}
$$

which is a closed subspace of $S^{(\mu ; \ell)}\left(\left(\mathbb{R}^{n} \times \mathbb{H}_{0}\right) \backslash\{0\} ; E, \tilde{E}\right)$.

3.22 Theorem. For every $\tau>0$ the mapping $T_{\tau}: a(\xi, \lambda) \mapsto a(\xi, \lambda+\tau)$ is continuous in the spaces

$$
T_{\tau}: S_{V}^{(\mu ; \ell)}\left(\left(\mathbb{R}^{n} \times \mathbb{H}_{0}\right) \backslash\{0\} ; E, \tilde{E}\right) \longrightarrow S_{V c l}^{\mu ; \ell}\left(\mathbb{R}^{n} \times \mathbb{H}_{0} ; E, \tilde{E}\right) .
$$

Moreover, for every 0-excision function $\chi \in C^{\infty}\left(\mathbb{R}^{n} \times \mathbb{H}_{0}\right)$ the following asymptotic expansion holds for $T_{\tau} a$ :

$$
T_{\tau} a \sim \sum_{k=0}^{\infty} \frac{\tau^{k}}{k !} \cdot \chi\left(\partial_{\lambda}^{k} a\right)
$$

This shows, in particular, that for the homogeneous component of order $\mu$ we have the identity $\left(T_{\tau} a\right)_{(\mu)}=a$.

In other words, the "principal symbol sequence" for Volterra symbols is topologically exact and splits:

$$
\begin{aligned}
0 \longrightarrow & S_{V c l}^{\mu-1 ; \ell}\left(\mathbb{R}^{n} \times \mathbb{H}_{0} ; E, \tilde{E}\right) \longrightarrow S_{V c l}^{\mu ; \ell}\left(\mathbb{R}^{n} \times \mathbb{H}_{0} ; E, \tilde{E}\right) \longrightarrow \\
& S_{V}^{(\mu ; \ell)}\left(\left(\mathbb{R}^{n} \times \mathbb{H}_{0}\right) \backslash\{0\} ; E, \tilde{E}\right) \longrightarrow 0 .
\end{aligned}
$$

The operator $T_{\tau}$ provides a splitting of this sequence. Analogous assertions hold in case of scales of Hilbert spaces involved. 
Proof. For $T_{\tau}$ acts continuously from $S_{V}^{(\mu ; \ell)}\left(\left(\mathbb{R}^{n} \times \mathbb{H}_{0}\right) \backslash\{0\} ; E, \tilde{E}\right)$ into the space $C^{\infty}\left(\mathbb{R}^{n} \times \mathbb{H}_{0}, \mathcal{L}(E, \tilde{E})\right) \cap \mathcal{A}\left(\mathbb{H}_{0}, C^{\infty}\left(\mathbb{R}^{n}, \mathcal{L}(E, \tilde{E})\right)\right)$, we only have to check that $T_{\tau} a \in S_{c l}^{\mu ; \ell}\left(\mathbb{R}^{n} \times \mathbb{H}_{0} ; E, \tilde{E}\right)$ in view of the closed graph theorem, as well as the asymptotic expansion for $T_{\tau} a$. For the assertion concerning the principal symbol sequence recall that the homogeneous components of a Volterra symbol are analytic in the interior of $\mathbb{H}_{0}$ according to Proposition 3.16.

Let $\alpha^{\prime}, \alpha^{\prime \prime} \in \mathbb{N}_{0}, \alpha:=\alpha^{\prime}+\alpha^{\prime \prime}$, and $\beta \in \mathbb{N}_{0}^{n}$. For every $N \in \mathbb{N}$ we have for $|\xi, \lambda|_{\ell}$ sufficiently large, using Taylor expansion,

$$
\begin{aligned}
& \left(\partial_{\lambda}+\partial_{\bar{\lambda}}\right)^{\alpha^{\prime}}\left(\partial_{\lambda}-\partial_{\bar{\lambda}}\right)^{\alpha^{\prime \prime}} \partial_{\xi}^{\beta}\left[a(\xi, \lambda+\tau)-\sum_{k=0}^{N-1} \frac{\tau^{k}}{k !} \cdot \chi(\xi, \lambda)\left(\partial_{\lambda}^{k} a\right)(\xi, \lambda)\right] \\
& =\partial_{\lambda}^{\alpha} \partial_{\xi}^{\beta}\left[a(\xi, \lambda+\tau)-\sum_{k=0}^{N-1} \frac{\tau^{k}}{k !} \cdot\left(\partial_{\lambda}^{k} a\right)(\xi, \lambda)\right] \\
& =\frac{\tau^{N}}{(N-1) !} \int_{0}^{1}(1-\theta)^{N-1}\left(\partial_{\lambda}^{N+\alpha} \partial_{\xi}^{\beta} a\right)(\xi, \lambda+\tau \theta) d \theta .
\end{aligned}
$$

The function $\left(\partial_{\lambda}^{N+\alpha} \partial_{\xi}^{\beta} a\right)$ is anisotropic homogeneous of degree $\mu-\ell(N+\alpha)-|\beta|$. Consequently, we are done if we show that for a smooth anisotropic homogeneous function $a$ of degree $\mu \in \mathbb{R}$ which is analytic in the interior of $\mathbb{H}_{0}$ we have

$$
\left\|\tilde{\kappa}_{\langle\xi, \lambda\rangle_{\ell}}^{-1} a(\xi, \lambda+\tau \theta) \kappa_{\langle\xi, \lambda\rangle_{\ell}}\right\|=O\left(\langle\xi, \lambda\rangle_{\ell}^{\mu}\right)
$$

for $|\xi, \lambda|_{\ell} \rightarrow \infty$, uniformly for $\theta \in[0,1]$. Let $M$ and $\tilde{M}$ be the constants in the norm estimates for the group-actions from (3.iv). Then we conclude, using (3.i), for $(\xi, \lambda) \neq 0$

$$
\begin{aligned}
& \left\|\tilde{\kappa}_{\langle\xi, \lambda\rangle_{\ell}}^{-1} a(\xi, \lambda+\tau \theta) \kappa_{\langle\xi, \lambda\rangle_{\ell}}\right\| \leq \operatorname{Const}\langle\tau \theta\rangle^{M+\tilde{M}} . \\
& \quad \cdot\left\|\tilde{\kappa}_{\langle\xi, \lambda+\tau \theta\rangle_{\ell}}^{-1} a\left(\langle\xi, \lambda+\tau \theta\rangle_{\ell} \frac{\xi}{\langle\xi, \lambda+\tau \theta\rangle_{\ell}},\langle\xi, \lambda+\tau \theta\rangle_{\ell}^{\ell} \frac{\lambda+\tau \theta}{\langle\xi, \lambda+\tau \theta\rangle_{\ell}^{\ell}}\right) \kappa_{\langle\xi, \lambda+\tau \theta\rangle_{\ell}}\right\| \\
& =\operatorname{Const}\langle\tau \theta\rangle^{M+\tilde{M}}\langle\xi, \lambda+\tau \theta\rangle_{\ell}^{\mu}\left\|a\left(\frac{\xi}{\langle\xi, \lambda+\tau \theta\rangle_{\ell}}, \frac{\lambda+\tau \theta}{\langle\xi, \lambda+\tau \theta\rangle_{\ell}^{\ell}}\right)\right\| \\
& \leq \operatorname{Const}\langle\tau \theta\rangle^{M+\tilde{M}+|\mu|}\langle\xi, \lambda\rangle_{\ell}^{\mu}\left\|a\left(\frac{\xi}{\langle\xi, \lambda+\tau \theta\rangle_{\ell}}, \frac{\lambda+\tau \theta}{\langle\xi, \lambda+\tau \theta\rangle_{\ell}^{\ell}}\right)\right\| .
\end{aligned}
$$

Observe that for $|\xi, \lambda|_{\ell} \geq 1$ and $\theta \in[0,1]$ we have, using (3.i), with a suitable 
constant $c>0$

$$
\begin{aligned}
1 & \geq\left|\frac{\xi}{\langle\xi, \lambda+\tau \theta\rangle_{\ell}}, \frac{\lambda+\tau \theta}{\langle\xi, \lambda+\tau \theta\rangle_{\ell}^{\ell}}\right|_{\ell}=\frac{1}{\langle\xi, \lambda+\tau \theta\rangle_{\ell}} \cdot|\xi, \lambda+\tau \theta|_{\ell} \\
& \geq c \cdot \frac{1}{\langle\xi, \lambda\rangle_{\ell}\langle\tau \theta\rangle} \cdot|\xi, \lambda+\tau \theta|_{\ell}=c \cdot \frac{1}{\left(1+|\xi, \lambda|_{\ell}^{2 \ell}\right)^{\frac{1}{2 \ell}}\langle\tau \theta\rangle} \cdot|\xi, \lambda+\tau \theta|_{\ell} \\
& \geq c \cdot \frac{1}{\left(2 \cdot|\xi, \lambda|_{\ell}^{2 \ell}\right)^{\frac{1}{2 \ell}} \cdot\langle\tau \theta\rangle} \cdot|\xi, \lambda|_{\ell} \geq c \cdot 2^{-\frac{1}{2 \ell}} \cdot\langle\tau\rangle^{-1}=: \tilde{c}>0 .
\end{aligned}
$$

Summing up, we thus obtain for $|\xi, \lambda|_{\ell} \geq 1$ for all $\theta \in[0,1]$

$$
\left\|\tilde{\kappa}_{\langle\xi, \lambda\rangle_{\ell}}^{-1} a(\xi, \lambda+\tau \theta) \kappa_{\langle\xi, \lambda\rangle_{\ell}}\right\| \leq\left(\text { Const } \cdot\langle\tau\rangle^{M+\tilde{M}+|\mu|} . \sup _{\tilde{c} \leq|\tilde{\xi}, \tilde{\lambda}|_{\ell} \leq 1}\|a(\tilde{\xi}, \tilde{\lambda})\|\right) \cdot\langle\xi, \lambda\rangle_{\ell}^{\mu} .
$$

This finishes the proof of the theorem.

\subsection{Holomorphic Volterra symbols}

3.23 Notation. For an interval $\emptyset \neq I \subseteq \mathbb{R}$ we shall use the notation

$$
\Gamma_{I}:=\{z \in \mathbb{C} ; \operatorname{Re}(z) \in I\}
$$

for the strip in the complex plane over $I$.

3.24 Definition. Let $E$ and $\tilde{E}$ be Hilbert spaces with group-actions $\left\{\kappa_{\varrho}\right\}$ and $\left\{\tilde{\kappa}_{\varrho}\right\}$, respectively. Moreover, let $z=\beta+i \tau \in \mathbb{C}$ be the splitting of $z \in \mathbb{C}$ in real and imaginary part. For $\mu \in \mathbb{R}$ define the Fréchet spaces

$$
\begin{aligned}
S_{O(c l)}^{\mu ; \ell}\left(\mathbb{R}^{n} \times \mathbb{C} ; E, \tilde{E}\right) & :=\mathcal{A}\left(\mathbb{C}, S^{\mu}\left(\mathbb{R}^{n} ; E, \tilde{E}\right)\right) \cap C^{\infty}\left(\mathbb{R}_{\beta}, S_{(c l)}^{\mu ; \ell}\left(\mathbb{R}^{n} \times \Gamma_{\beta} ; E, \tilde{E}\right)\right), \\
S_{V, O(c l)}^{\mu ; \ell}\left(\mathbb{R}^{n} \times \mathbb{C} ; E, \tilde{E}\right) & :=S_{O(c l)}^{\mu ; \ell}\left(\mathbb{R}^{n} \times \mathbb{C} ; E, \tilde{E}\right) \cap S_{V(c l)}^{\mu ; \ell}\left(\mathbb{R}^{n} \times \mathbb{H}_{0} ; E, \tilde{E}\right)
\end{aligned}
$$

with the induced topologies. Analogously, we define the corresponding symbol spaces when we deal with scales of Hilbert spaces.

3.25 Proposition. Let $\emptyset \neq I \subseteq \mathbb{R}$ be an open interval and $\mu \in \mathbb{R}$. Let

$$
\begin{aligned}
& \ell_{\text {loc }}^{\infty} \mathcal{A}_{(c l)}^{\mu ; \ell}(E, \tilde{E}):=\left\{a \in \mathcal{A}\left(\Gamma_{I}, S^{\mu}\left(\mathbb{R}^{n} ; E, \tilde{E}\right)\right) ;\left.a\right|_{\Gamma_{\beta}} \in S_{(c l)}^{\mu ; \ell}\left(\mathbb{R}^{n} \times \Gamma_{\beta} ; E, \tilde{E}\right)\right. \\
&\text { locally uniformly for } \beta \in I\} \\
& C^{\infty} \mathcal{A}_{(c l)}^{\mu ; \ell}(E, \tilde{E}):=\left\{a \in \mathcal{A}\left(\Gamma_{I}, S^{\mu}\left(\mathbb{R}^{n} ; E, \tilde{E}\right)\right) ; a \in C^{\infty}\left(I_{\beta}, S_{(c l)}^{\mu ; \ell}\left(\mathbb{R}^{n} \times \Gamma_{\beta} ; E, \tilde{E}\right)\right)\right\}
\end{aligned}
$$

endowed with their natural Fréchet topologies. Observe that for $I=\mathbb{R}$ we recover $S_{O(c l)}^{\mu ; \ell}\left(\mathbb{R}^{n} \times \mathbb{C} ; E, \tilde{E}\right)=C^{\infty} \mathcal{A}_{(c l)}^{\mu ; \ell}(E, \tilde{E})$. 
a) The embedding $\iota: C^{\infty} \mathcal{A}_{(c l)}^{\mu ; \ell}(E, \tilde{E}) \hookrightarrow \ell_{l o c}^{\infty} \mathcal{A}_{(c l)}^{\mu ; \ell}(E, \tilde{E})$ is onto and provides an isomorphism between these spaces.

b) The complex derivative is a linear and continuous operator in the spaces $\partial_{z}$ : $\ell_{l o c}^{\infty} \mathcal{A}_{(c l)}^{\mu ; \ell}(E, \tilde{E}) \rightarrow \ell_{l o c}^{\infty} \mathcal{A}_{(c l)}^{\mu-\ell ; \ell}(E, \tilde{E})$.

c) Given $a \in \ell_{\text {loc }}^{\infty} \mathcal{A}_{(c l)}^{\mu ; \ell}(E, \tilde{E})$, we have the following asymptotic expansion for $\left.a\right|_{\Gamma_{\beta_{0}}}$ in terms of $\left.a\right|_{\Gamma_{\beta}}$ for every $\beta_{0}, \beta \in I$ which depends smoothly on $\left(\beta_{0}, \beta\right) \in I \times I$ :

$$
\left.\left.a\right|_{\Gamma_{\beta_{0}}} \sim \sum_{k=0}^{\infty} \frac{\left(\beta_{0}-\beta\right)^{k}}{k !}\left(\partial_{z}^{k} a\right)\right|_{\Gamma_{\beta}} .
$$

d) For arbitrary $\beta \in \mathbb{R}$ we have $S_{V, O(c l)}^{\mu ; \ell}\left(\mathbb{R}^{n} \times \mathbb{C} ; E, \tilde{E}\right) \hookrightarrow S_{V(c l)}^{\mu ; \ell}\left(\mathbb{R}^{n} \times \mathbb{H}_{\beta} ; E, \tilde{E}\right)$. If $a \in S_{V, O(c l)}^{\mu ; \ell}\left(\mathbb{R}^{n} \times \mathbb{C} ; E, \tilde{E}\right)$, then we have $\left.a\right|_{\mathbb{H}_{\beta}} \in S_{V(c l)}^{\mu ; \ell}\left(\mathbb{R}^{n} \times \mathbb{H}_{\beta} ; E, \tilde{E}\right)$ as a smooth function of $\beta \in \mathbb{R}$, and the asymptotic expansion

$$
\left.\left.a\right|_{\mathbb{H}_{\beta_{0}}} \sim \sum_{k=0}^{\infty} \frac{\left(\beta_{0}-\beta\right)^{k}}{k !}\left(\partial_{z}^{k} a\right)\right|_{\mathbb{H}_{\beta}}
$$

is valid, which depends smoothly on $\left(\beta_{0}, \beta\right) \in \mathbb{R} \times \mathbb{R}$.

e) For $\beta \in I$ and $\mu \geq \mu^{\prime}$ the identity

$$
\ell_{\text {loc }}^{\infty} \mathcal{A}^{\mu ; \ell}(E, \tilde{E}) \cap S_{(c l)}^{\mu^{\prime} ; \ell}\left(\mathbb{R}^{n} \times \Gamma_{\beta} ; E, \tilde{E}\right)=\ell_{l o c}^{\infty} \mathcal{A}_{(c l)}^{\mu^{\prime} ; \ell}(E, \tilde{E})
$$

holds algebraically and topologically.

f) For $\beta \in \mathbb{R}$ and $\mu \geq \mu^{\prime}$ the identity

$$
S_{V, O}^{\mu ; \ell}\left(\mathbb{R}^{n} \times \mathbb{C} ; E, \tilde{E}\right) \cap S_{V(c l)}^{\mu^{\prime} ; \ell}\left(\mathbb{R}^{n} \times \mathbb{H}_{\beta} ; E, \tilde{E}\right)=S_{V, O(c l)}^{\mu^{\prime} ; \ell}\left(\mathbb{R}^{n} \times \mathbb{C} ; E, \tilde{E}\right)
$$

holds algebraically and topologically.

From the expansions in c) and d) we see, that in the classical cases the homogeneous principal symbols of the restrictions do not depend on the particular weight line or half-plane.

Proof. Let $a \in \ell_{\mathrm{loc}}^{\infty} \mathcal{A}_{(c l)}^{\mu ; \ell}(E, \tilde{E})$. Carrying out a Taylor expansion we may write for $\beta, \beta_{0} \in I$ and $\tau \in \mathbb{R}$, for every $N \in \mathbb{N}$ :

$$
\begin{aligned}
a\left(\beta_{0}+i \tau\right)=\sum_{k=0}^{N-1} & \frac{\left(\beta_{0}-\beta\right)^{k}}{k !}\left(\partial_{z}^{k} a\right)(\beta+i \tau) \\
& +\underbrace{\frac{\left(\beta_{0}-\beta\right)^{N}}{(N-1) !} \int_{0}^{1}(1-\theta)^{N-1}\left(\partial_{z}^{N} a\right)\left(\beta+\theta\left(\beta_{0}-\beta\right)+i \tau\right) d \theta}_{=: r_{N}\left(\beta_{0}, \beta, \tau\right)} .
\end{aligned}
$$


Observe that $\partial_{z} a=D_{\tau} a$, and thus for $N=2$ equation (1) gives

$$
\begin{aligned}
\frac{a\left(\beta_{0}+i \tau\right)-a(\beta+i \tau)}{\beta_{0}-\beta} & -\left(\partial_{z} a\right)(\beta+i \tau) \\
& =\left(\beta_{0}-\beta\right) \underbrace{\int_{0}^{1}(1-\theta)\left(D_{\tau}^{2} a\right)\left(\beta+\theta\left(\beta_{0}-\beta\right)+i \tau\right) d \theta} .
\end{aligned}
$$

By assumption $(*)$ remains bounded in $S_{(c l)}^{\mu ; \ell}\left(\mathbb{R}^{n} \times \mathbb{R}_{\tau} ; E, \tilde{E}\right)$ for $\beta_{0} \rightarrow \beta$, and consequently we see that there exists $\left(\partial_{\beta} a\right)(\beta+i \cdot)=\left(\partial_{z} a\right)(\beta+i \cdot)$ for $\beta \in I$ in $S_{(c l)}^{\mu ; \ell}\left(\mathbb{R}^{n} \times \Gamma_{\beta} ; E, \tilde{E}\right)$. For $\partial_{z} a=D_{\tau} a \in \ell_{\mathrm{loc}}^{\infty} \mathcal{A}_{(c l)}^{\mu-\ell ; \ell}(E, \tilde{E})$ we obtain by induction the assertions a) and b) of the proposition in view of the closed graph theorem.

If $a \in S_{V, O}^{\mu ; \ell}\left(\mathbb{R}^{n} \times \mathbb{C} ; E, \tilde{E}\right)$, then $\left.a\right|_{\mathbb{H}_{\beta}} \in S_{V}^{\mu ; \ell}\left(\mathbb{R}^{n} \times \mathbb{H}_{\beta} ; E, \tilde{E}\right)$ locally uniformly for $\beta \in \mathbb{R}$ with continuous embedding. This follows at once from (3.i) and (3.iv), see also Proposition 3.20. For $\tau \in \mathbb{H}_{-}$and $\beta, \beta_{0} \in \mathbb{R}$ we conclude from formula (1) and (2) with the same reasoning as above that $\left.a\right|_{\mathbb{H}_{\beta}} \in S_{V}^{\mu ; \ell}\left(\mathbb{R}^{n} \times \mathbb{H}_{\beta} ; E, \tilde{E}\right)$ as a smooth function of $\beta \in \mathbb{R}$.

Let us now show the claim about the asymptotic expansion in c) and d). From the already proven part of the proposition we conclude that for $N \in \mathbb{N}$ the function $\left(\partial_{z}^{N} a\right)\left(\beta+\theta\left(\beta_{0}-\beta\right)+i \tau\right)$ may be regarded as a continuous function of $\theta \in[0,1]$ taking values in the space $C^{\infty}\left(I_{\beta_{0}} \times I_{\beta}, S^{\mu-N \ell ; \ell}\left(\mathbb{R}^{n} \times \mathbb{R}_{\tau} ; E, \tilde{E}\right)\right)$, and thus the remainder $r_{N}\left(\beta_{0}, \beta, \tau\right)$ in (1) is a smooth function of $\left(\beta_{0}, \beta\right) \in I \times I$ taking values in $S^{\mu-N \ell ; \ell}\left(\mathbb{R}^{n} \times \mathbb{R}_{\tau} ; E, \tilde{E}\right)$. This shows the validity of the asymptotic expansion in c). The expansion in d) follows analogously, but now we deal with Volterra symbols with respect to the variable $\tau \in \mathbb{H}_{-}$. From this we also obtain the missing part of d) in the classical case.

The assertions in e) and f) follow from the expansions in c) and d). This finishes the proof of the proposition.

3.26 Remark. Assume that we are given symbols $a_{k} \in S_{(V,) O}^{\mu_{k} ; \ell}\left(\mathbb{R}^{n} \times \mathbb{C} ; E, \tilde{E}\right)$, as well as $a \in S_{(V,) O}^{\bar{\mu} ; \ell}\left(\mathbb{R}^{n} \times \mathbb{C} ; E, \tilde{E}\right)$, where $\left(\mu_{k}\right) \subseteq \mathbb{R}$ with $\mu_{k} \rightarrow-\infty$ as $k \rightarrow \infty$, and $\bar{\mu}=\max _{k \in \mathbb{N}_{0}} \mu_{k}$. Moreover, assume that for some $\beta \in \mathbb{R}$ we have $\left.\left.a\right|_{\Gamma_{\beta}} \sim \sum_{k=0}^{\infty} a_{k}\right|_{\Gamma_{\beta}}$, or $\left.\left.a\right|_{\mathbb{H}_{\beta}} \widetilde{V} \sum_{k=0}^{\infty} a_{k}\right|_{\mathbb{H}_{\beta}}$, in the sense of Definition 3.7 or Definition 3.17 , respectively.

Then Proposition 3.25 implies that $a \underset{(V)}{\sim} \sum_{k=0}^{\infty} a_{k}$ in the sense of holomorphic (Volterra) symbols, i. e., the remainders already belong to the class of holomorphic (Volterra) symbols. In particular, for holomorphic symbols there is no proper refinement of the notion of asymptotic expansion. 


\section{The calculus of Volterra Mellin operators}

\subsection{General Volterra Mellin operators}

4.1 Definition. Let $E$ and $\tilde{E}$ be Hilbert spaces endowed with strongly continuous group-actions $\left\{\kappa_{\varrho}\right\}$ and $\left\{\tilde{\kappa}_{\varrho}\right\}$, respectively. We define for $\mu \in \mathbb{R}$ the spaces of $\left(r, r^{\prime}\right)$ resp. $r$-dependent (classical) parameter-dependent Mellin symbols with respect to the weight $\gamma \in \mathbb{R}$ and parameter-space $\mathbb{R}^{n}$ as

$$
M_{\gamma} S_{(c l)}^{\mu ; \ell}\left(\left(\mathbb{R}_{+}\right)^{q} \times \mathbb{R}^{n} \times \Gamma_{\frac{1}{2}-\gamma} ; E, \tilde{E}\right):=C_{B}^{\infty}\left(\left(\mathbb{R}_{+}\right)^{q}, S_{(c l)}^{\mu ; \ell}\left(\mathbb{R}^{n} \times \Gamma_{\frac{1}{2}-\gamma} ; E, \tilde{E}\right)\right)
$$

for $q=1,2$. Correspondingly, the spaces of (classical) Volterra Mellin symbols of order $\mu$ with respect to the weight $\gamma \in \mathbb{R}$ are defined as

$$
M_{\gamma} S_{V(c l)}^{\mu ; \ell}\left(\left(\mathbb{R}_{+}\right)^{q} \times \mathbb{R}^{n} \times \mathbb{H}_{\frac{1}{2}-\gamma} ; E, \tilde{E}\right):=C_{B}^{\infty}\left(\left(\mathbb{R}_{+}\right)^{q}, S_{V(c l)}^{\mu ; \ell}\left(\mathbb{R}^{n} \times \mathbb{H}_{\frac{1}{2}-\gamma} ; E, \tilde{E}\right)\right)
$$

for $q=1,2$. Analogously, we obtain the spaces of order $-\infty$ with respect to the weight $\gamma \in \mathbb{R}$. All these spaces carry Fréchet topologies in a canonical way. With the same conventions as in Definition 3.4 we also have the (Volterra) Mellin symbol spaces when we deal with scales of Hilbert spaces instead of single Hilbert spaces only.

From Proposition 3.16 we see that the operator of restriction of the half-plane $\mathbb{H}_{\frac{1}{2}-\gamma}$ to the weight line $\Gamma_{\frac{1}{2}-\gamma}$ induces continuous embeddings

$$
M_{\gamma} S_{V(c l)}^{\mu ; \ell}\left(\mathbb{R}_{+} \times \mathbb{R}^{n} \times \mathbb{H}_{\frac{1}{2}-\gamma} ; E, \tilde{E}\right) \hookrightarrow M_{\gamma} S_{(c l)}^{\mu ; \ell}\left(\mathbb{R}_{+} \times \mathbb{R}^{n} \times \Gamma_{\frac{1}{2}-\gamma} ; E, \tilde{E}\right)
$$

4.2 Definition. Let $E$ and $\tilde{E}$ be Hilbert spaces with group-actions $\left\{\kappa_{\varrho}\right\}$ and $\left\{\tilde{\kappa}_{\varrho}\right\}$, respectively, and let $\mu \in \mathbb{R}$. With a Mellin double-symbol $a \in M_{\gamma} S^{\mu ; \ell}\left(\mathbb{R}_{+} \times\right.$ $\left.\mathbb{R}_{+} \times \mathbb{R}^{n} \times \Gamma_{\frac{1}{2}-\gamma} ; E, \tilde{E}\right)$ we associate a family of Mellin pseudodifferential operators $\operatorname{op}_{M}^{\gamma}(a)(\xi) \in \mathcal{L}\left(\mathcal{T}_{\gamma}\left(\mathbb{R}_{+}, E\right), \mathcal{T}_{\gamma}\left(\mathbb{R}_{+}, \tilde{E}\right)\right)$ for $\xi \in \mathbb{R}^{n}$ by means of the following Mellin oscillatory integral:

$$
\begin{aligned}
\left(\operatorname{op}_{M}^{\gamma}(a)(\xi) u\right)(r): & =\frac{1}{2 \pi i} \int_{\Gamma_{\frac{1}{2}-\gamma}} \int_{\mathbb{R}_{+}}\left(\frac{r}{r^{\prime}}\right)^{-z} a\left(r, r^{\prime}, \xi, z\right) u\left(r^{\prime}\right) \frac{d r^{\prime}}{r^{\prime}} d z \\
& =\int_{\mathbb{R}_{\mathbb{R}_{+}}} \int_{r^{\frac{1}{2}-\gamma+i \tau}} a\left(r, r r^{\prime}, \xi, \frac{1}{2}-\gamma+i \tau\right) u\left(r r^{\prime}\right) \frac{d r^{\prime}}{r^{\prime}} d \tau .
\end{aligned}
$$

Taking into account the operator $S_{\gamma}$ and its inverse from (2.i) and (2.ii) we see that we may write

$$
\mathrm{op}_{M}^{\gamma}(a)(\xi)=S_{\gamma}^{-1} \mathrm{op}_{t}\left(a_{\gamma}\right)(\xi) S_{\gamma}
$$


as operators on $\mathcal{T}_{\gamma}\left(\mathbb{R}_{+}, E\right)$, where the (Fourier) double-symbol $a_{\gamma} \in C_{b}^{\infty}(\mathbb{R} \times$ $\left.\mathbb{R}, S^{\mu ; \ell}\left(\mathbb{R}^{n} \times \mathbb{R} ; E, \tilde{E}\right)\right)$ is given as

$$
a_{\gamma}\left(t, t^{\prime}, \xi, \tau\right)=a\left(e^{-t}, e^{-t^{\prime}}, \xi, \frac{1}{2}-\gamma+i \tau\right),
$$

and $\mathrm{op}_{t}\left(a_{\gamma}\right)(\xi): \mathcal{S}(\mathbb{R}, E) \longrightarrow \mathcal{S}(\mathbb{R}, \tilde{E})$ is a usual pseudodifferential operator based on the Fourier transform (Kohn-Nirenberg quantization). From (4.i) we thus see that the theory of Mellin pseudodifferential operators can be carried over to some extent from the setting of operators based on the Fourier transform, and vice versa.

4.3 Theorem. Let $a \in M_{\gamma} S_{(c l)}^{\mu ; \ell}\left(\mathbb{R}_{+} \times \mathbb{R}_{+} \times \mathbb{R}^{n} \times \Gamma_{\frac{1}{2}-\gamma} ; E, \tilde{E}\right)$. Then there exist unique Mellin left- and right-symbols $a_{L}(r, \xi, z), a_{R}\left(r^{\prime}, \xi, z\right) \in M_{\gamma} S_{(c l)}^{\mu, \ell}\left(\mathbb{R}_{+} \times \mathbb{R}^{n} \times\right.$ $\left.\Gamma_{\frac{1}{2}-\gamma} ; E, \tilde{E}\right)$ such that $o p_{M}^{\gamma}(a)(\xi)=o p_{M}^{\gamma}\left(a_{L}\right)(\xi)=o p_{M}^{\gamma}\left(a_{R}\right)(\xi)$ as operators on $\mathcal{T}_{\gamma}\left(\mathbb{R}_{+}, E\right)$.

These symbols are given by the following Mellin oscillatory integrals:

$$
\begin{aligned}
a_{L}(r, \xi, z) & =\iint_{\mathbb{R}_{+}} s^{i \eta} a(r, s r, \xi, z+i \eta) \frac{d s}{s} d \eta, \\
a_{R}\left(r^{\prime}, \xi, z\right) & =\iint_{\mathbb{R}_{\mathbb{R}_{+}}} s^{i \eta} a\left(s r^{\prime}, r^{\prime}, \xi, z-i \eta\right) \frac{d s}{s} d \eta .
\end{aligned}
$$

The mappings $a \mapsto a_{L}$ and $a \mapsto a_{R}$ are continuous. Moreover, we have the asymptotic expansions

$$
\begin{aligned}
a_{L}(r, \xi, z) & \left.\sim \sum_{k=0}^{\infty} \frac{1}{k !} D_{\tau}^{k}\left(-r^{\prime} \partial_{r^{\prime}}\right)^{k} a\left(r, r^{\prime}, \xi, z\right)\right|_{r^{\prime}=r}, \\
a_{R}\left(r^{\prime}, \xi, z\right) & \left.\sim \sum_{k=0}^{\infty} \frac{1}{k !}(-1)^{k} D_{\tau}^{k}\left(-r \partial_{r}\right)^{k} a\left(r, r^{\prime}, \xi, z\right)\right|_{r=r^{\prime}} .
\end{aligned}
$$

If $a \in M_{\gamma} S_{V(c l)}^{\mu, \ell}\left(\mathbb{R}_{+} \times \mathbb{R}_{+} \times \mathbb{R}^{n} \times \mathbb{H}_{\frac{1}{2}-\gamma} ; E, \tilde{E}\right)$ then also $a_{L}, a_{R} \in M_{\gamma} S_{V(c l)}^{\mu ; \ell}\left(\mathbb{R}_{+} \times\right.$ $\left.\mathbb{R}^{n} \times \mathbb{H}_{\frac{1}{2}-\gamma} ; E, \tilde{E}\right)$, and the mappings $a \mapsto a_{L}$ and $a \mapsto a_{R}$ are continuous with respect to the topology of the Volterra Mellin symbol spaces. In this case we have the asymptotic expansions

$$
\begin{aligned}
& \left.a_{L}(r, \xi, z) \underset{V}{\sim} \sum_{k=0}^{\infty} \frac{1}{k !} \partial_{z}^{k}\left(-r^{\prime} \partial_{r^{\prime}}\right)^{k} a\left(r, r^{\prime}, \xi, z\right)\right|_{r^{\prime}=r}, \\
& \left.a_{R}\left(r^{\prime}, \xi, z\right) \underset{V}{\sim} \sum_{k=0}^{\infty} \frac{1}{k !}(-1)^{k} \partial_{z}^{k}\left(-r \partial_{r}\right)^{k} a\left(r, r^{\prime}, \xi, z\right)\right|_{r=r^{\prime}}
\end{aligned}
$$

in the Volterra sense. 
Proof. In view of (4.i) the assertions of the theorem in the non-Volterra case concerning the existence of unique Mellin left- and right-symbols, as well as their oscillatory integral formulas and the asymptotic expansions, follow from the calculus of standard pseudodifferential operators with symbols that satisfy global estimates, see, e. g., Kumano-go [27], Shubin [48] (in the scalar case), or Dorschfeldt, Grieme, and Schulze [8] (in case of operator-valued symbols). In the framework of Mellin operators notice, in particular, the work of Lewis and Parenti [28], and in case of operator-valued symbols the works of Dorschfeldt [7], Dorschfeldt and Schulze [9], and Seiler [46], [47].

What remains to show are the assertions in the Volterra case. In order to do so, we only need to prove that the left- and right-symbols, which we obtain from the first part of the theorem, are Volterra symbols (more precisely that they originate from Volterra symbols via restriction to the weight line), and that the asymptotic expansions hold in the Volterra sense. From these expansions we then see that the class of classical Volterra symbols remains preserved, and the asserted continuities follow from the closed graph theorem. We carry out the proof for the left-symbol only, because the proof for the right-symbol is analogous.

Let $a \in M_{\gamma} S_{V}^{\mu ; \ell}\left(\mathbb{R}_{+} \times \mathbb{R}_{+} \times \mathbb{R}^{n} \times \mathbb{H}_{\frac{1}{2}-\gamma} ; E, \tilde{E}\right)$ be a Mellin double-symbol, and

$$
a_{L}(r, \xi, z)=\int_{\mathbb{R}_{\mathbb{R}_{+}}} s^{i \eta} a(r, s r, \xi, z+i \eta) \frac{d s}{s} d \eta
$$

$z \in \Gamma_{\frac{1}{2}-\gamma}$, the associated left-symbol to $\left.a\right|_{\Gamma_{\frac{1}{2}-\gamma}}$. Note first that the oscillatory integral formula (1) does not only make sense for $z \in \Gamma_{\frac{1}{2}-\gamma}$ but also for $z \in \mathbb{H}_{\frac{1}{2}-\gamma}$, which provides an extension of $a_{L}(r, \xi, z)$ from the weight line to the half-plane. More precisely, the function $a(r, s r, \xi, z+i \eta)$ may be regarded as a smooth function of $(r, \xi, z) \in \mathbb{R}_{+} \times \mathbb{R}^{n} \times \mathbb{H}_{\frac{1}{2}-\gamma}$ which is analytic in the interior of $\mathbb{H}_{\frac{1}{2}-\gamma}$, taking values in the operator-valued Mellin amplitude functions in the variables $(s, \eta) \in \mathbb{R}_{+} \times \mathbb{R}$, since the symbol $a$ is a Volterra Mellin double-symbol by assumption. This shows that $a_{L}(r, \xi, z)$ is a smooth function of $(r, \xi, z) \in \mathbb{R}_{+} \times \mathbb{R}^{n} \times \mathbb{H}_{\frac{1}{2}-\gamma}$ which is analytic in the interior of $\mathbb{H}_{\frac{1}{2}-\gamma}$. We need to prove that the symbol estimates for $a_{L}(r, \xi, z)$ extend to the half-plane.

First observe that we may interchange the differentiation with respect to $\xi \in \mathbb{R}^{n}$ and $z \in \mathbb{H}_{\frac{1}{2}-\gamma}$ with the oscillatory integral in (1). Thus for $\alpha \in \mathbb{N}_{0}^{n}$ and $\beta \in \mathbb{N}_{0}$ we obtain

$$
\left(\partial_{\xi}^{\alpha} \partial_{z}^{\beta} a_{L}\right)(r, \xi, z)=\int_{\mathbb{R}_{\mathbb{R}_{+}}} \int^{i \eta}\left(\partial_{\xi}^{\alpha} \partial_{z}^{\beta} a\right)(r, s r, \xi, z+i \eta) \frac{d s}{s} d \eta,
$$

where $\partial_{\xi}^{\alpha} \partial_{z}^{\beta} a \in M_{\gamma} S_{V}^{\mu-|\alpha|-\ell|\beta| ; \ell}\left(\mathbb{R}_{+} \times \mathbb{R}_{+} \times \mathbb{R}^{n} \times \mathbb{H}_{\frac{1}{2}-\gamma} ; E, \tilde{E}\right)$, i. e., $\partial_{\xi}^{\alpha} \partial_{z}^{\beta} a_{L}$ is the associated left-symbol to $\partial_{\xi}^{\alpha} \partial_{z}^{\beta} a$. Consequently, we simply have to prove that for 
all $k \in \mathbb{N}_{0}$ we have

$$
\left\|\tilde{\kappa}_{\langle\xi, \zeta\rangle_{\ell}}^{-1}\left(-r \partial_{r}\right)^{k} a_{L}\left(r, \xi, \frac{1}{2}-\gamma+\zeta\right) \kappa_{\langle\xi, \zeta\rangle_{\ell}}\right\|=O\left(\langle\xi, \zeta\rangle_{\ell}^{\mu}\right)
$$

for $(\xi, \zeta) \in \mathbb{R}^{n} \times \mathbb{H}_{0}$ as $|\xi, \zeta|_{\ell} \rightarrow \infty$, uniformly for $r \in \mathbb{R}_{+}$.

For $k \in \mathbb{N}_{0}$ we have according to (1)

$$
\left(-r \partial_{r}\right)^{k} a_{L}\left(r, \xi, \frac{1}{2}-\gamma+\zeta\right)=\int_{\mathbb{R}_{\mathbb{R}_{+}}} s^{i \eta}\left(-r \partial_{r}\right)^{k} a\left(r, s r, \xi, \frac{1}{2}-\gamma+\zeta+i \eta\right) \frac{d s}{s} d \eta
$$

where we may write

$$
\begin{aligned}
& \left(-r \partial_{r}\right)^{k} a\left(r, s r, \xi, \frac{1}{2}-\gamma+\zeta+i \eta\right)= \\
& \quad \sum_{j=0}^{k}\left(\begin{array}{l}
k \\
j
\end{array}\right)\left[\left(-r \partial_{r}\right)^{j}\left(-r^{\prime} \partial_{r^{\prime}}\right)^{k-j} a\right]\left(r, s r, \xi, \frac{1}{2}-\gamma+\zeta+i \eta\right) .
\end{aligned}
$$

Consequently, the proof reduces to consider the case $k=0$. Regularizing the oscillatory integral (1) yields

$$
\begin{aligned}
& a_{L}\left(r, \xi, \frac{1}{2}-\gamma+\zeta\right)=\iint_{\mathbb{R}_{\mathbb{R}_{+}}} s^{i \eta} a\left(r, s r, \xi, \frac{1}{2}-\gamma+\zeta+i \eta\right) \frac{d s}{s} d \eta=\iint_{\mathbb{R}_{\mathbb{R}_{+}}} s^{i \eta}\langle\log s\rangle^{-2} \\
& \underbrace{\left(1-\partial_{\eta}^{2}\right)\left[\langle\eta\rangle^{-2 \nu}\left(\left(1-\left(-r^{\prime} \partial_{r^{\prime}}\right)^{2}\right)^{\nu} a\right)\left(r, s r, \xi, \frac{1}{2}-\gamma+\zeta+i \eta\right)\right]}_{(*)} \frac{d s}{s} d \eta,
\end{aligned}
$$

where $\nu \in \mathbb{N}_{0}$ is chosen sufficiently large. $(*)$ is a linear combination of terms of the form

$$
\left(D_{\eta}^{i}\langle\eta\rangle^{-2 \nu}\right) \cdot\left(\left(1-\left(-r^{\prime} \partial_{r^{\prime}}\right)^{2}\right)^{\nu} \partial_{\zeta}^{j} a\right)\left(r, s r, \xi, \frac{1}{2}-\gamma+\zeta+i \eta\right)
$$

for $0 \leq i, j \leq 2$. For $a$ is a Volterra Mellin double-symbol by assumption we conclude, using (3.i) and the constants $M, \tilde{M}$ of the norm estimates for the groupactions from (3.iv), that

$$
\begin{aligned}
& \left\|\tilde{\kappa}_{\langle\xi, \zeta\rangle_{\ell}}^{-1}\left(\left(1-\left(-r^{\prime} \partial_{r^{\prime}}\right)^{2}\right)^{\nu} \partial_{\zeta}^{j} a\right)\left(r, s r, \xi, \frac{1}{2}-\gamma+\zeta+i \eta\right) \kappa_{\langle\xi, \zeta\rangle_{\ell}}\right\| \leq\left\|\tilde{\kappa}_{\langle\xi, \zeta+i \eta\rangle_{\ell}\langle\xi, \zeta\rangle_{\ell}^{-1}}\right\| \\
& \left\|\tilde{\kappa}_{\langle\xi, \zeta+i \eta\rangle_{\ell}}^{-1}\left(\left(1-\left(-r^{\prime} \partial_{r^{\prime}}\right)^{2}\right)^{\nu} \partial_{\zeta}^{j} a\right)\left(r, s r, \xi, \frac{1}{2}-\gamma+\zeta+i \eta\right) \kappa_{\langle\xi, \zeta+i \eta\rangle_{\ell}}\right\| \\
& \left\|\kappa_{\langle\xi, \zeta+i \eta\rangle_{\ell}^{-1}\langle\xi, \zeta\rangle_{\ell}}\right\| \leq \mathrm{Const}\langle\eta\rangle^{\frac{M+\tilde{M}}{\ell}}\langle\xi, \zeta+i \eta\rangle_{\ell}^{\mu-j \ell} \leq \mathrm{Const}\langle\eta\rangle^{\frac{M+\tilde{M}+|\mu|}{\ell}}\langle\xi, \zeta\rangle_{\ell}^{\mu} .
\end{aligned}
$$

Now we see that if we choose $\nu>\frac{M+\tilde{M}+|\mu|}{2 \ell}+1$ the desired estimates indeed hold for $a_{L}$. Summing up, we have shown that $a_{L}$ is a Volterra Mellin symbol. It 
remains to prove the validity of the asymptotic expansion for $a_{L}$ in terms of $a$ in the Volterra sense.

Employing a Taylor expansion in $\eta=0$ we may write for each $N \in \mathbb{N}$

$$
\begin{aligned}
a(r, s r, \xi, z+i \eta)= & \sum_{k=0}^{N-1} \frac{1}{k !}\left(\partial_{z}^{k} a\right)(r, s r, \xi, z)(i \eta)^{k} \\
& +(i \eta)^{N} \int_{0}^{1} \frac{(1-\theta)^{N-1}}{(N-1) !}\left(\partial_{z}^{N-1} a\right)(r, s r, \xi, z+i \theta \eta) d \theta .
\end{aligned}
$$

The terms of the Taylor polynomial are Mellin amplitude functions in the variables $(s, \eta)$. Moreover, the integrand in the Taylor remainder can be viewed as a continuous function of $\theta \in[0,1]$ with values in the Mellin amplitude functions in $(s, \eta)$. Integrating by parts in the Mellin oscillatory integral formula (1) for $a_{L}$ and interchanging integrals of the remainder gives

$$
a_{L}(r, \xi, z)=\left.\sum_{k=0}^{N-1} \frac{1}{k !} \partial_{z}^{k}\left(-r^{\prime} \partial_{r^{\prime}}\right)^{k} a\left(r, r^{\prime}, \xi, z\right)\right|_{r^{\prime}=r}+r_{L, N}(r, \xi, z),
$$

where

$$
r_{L, N}(r, \xi, z)=\int_{0}^{1} \frac{(1-\theta)^{N-1}}{(N-1) !} \underbrace{\int_{\mathbb{R}^{R}} \int_{\mathbb{R}_{+}} s^{i \eta}\left(\partial_{z}^{N}\left(-r^{\prime} \partial_{r^{\prime}}\right)^{N} a\right)(r, s r, \xi, z+i \theta \eta) \frac{d s}{s} d \eta}_{(* *)} d \theta .
$$

Similar arguments as above show that $(* *)$ may be viewed as a continuous function of $\theta \in[0,1]$ taking values in the Volterra Mellin symbols, and thus we have

$$
\begin{aligned}
\left.\frac{1}{k !} \partial_{z}^{k}\left(-r^{\prime} \partial_{r^{\prime}}\right)^{k} a\left(r, r^{\prime}, \xi, z\right)\right|_{r^{\prime}=r} & \in M_{\gamma} S_{V}^{\mu-k \ell ; \ell}\left(\mathbb{R}_{+} \times \mathbb{R}^{n} \times \mathbb{H}_{\frac{1}{2}-\gamma} ; E, \tilde{E}\right), \\
r_{L, N}(r, \xi, z) & \in M_{\gamma} S_{V}^{\mu-N \ell ; \ell}\left(\mathbb{R}_{+} \times \mathbb{R}^{n} \times \mathbb{H}_{\frac{1}{2}-\gamma} ; E, \tilde{E}\right),
\end{aligned}
$$

which yields the desired asymptotic expansion.

4.4 Definition. For $\gamma \in \mathbb{R}$ define

$$
\begin{aligned}
M_{\gamma} L_{(c l)}^{\mu ; \ell}\left(\mathbb{R}_{+} ; \mathbb{R}^{n} ; E, \tilde{E}\right) & :=\left\{\operatorname{op}_{M}^{\gamma}(a)(\xi) ; a \in M_{\gamma} S_{(c l)}^{\mu ; \ell}\left(\mathbb{R}_{+} \times \mathbb{R}^{n} \times \Gamma_{\frac{1}{2}-\gamma} ; E, \tilde{E}\right)\right\} \\
M_{\gamma} L_{V(c l)}^{\mu ; \ell}\left(\mathbb{R}_{+} ; \mathbb{R}^{n} ; E, \tilde{E}\right) & :=\left\{\operatorname{op}_{M}^{\gamma}(a)(\xi) ; a \in M_{\gamma} S_{V(c l)}^{\mu ; \ell}\left(\mathbb{R}_{+} \times \mathbb{R}^{n} \times \mathbb{H}_{\frac{1}{2}-\gamma} ; E, \tilde{E}\right)\right\}
\end{aligned}
$$

In view of Theorem 4.3 we conclude that $\operatorname{op}_{M}^{\gamma}(\cdot)(\xi)$ provides an isomorphism between these spaces and the corresponding (left-) symbol spaces. Via that isomorphism we carry over the topologies which turns the operator spaces into Fréchet spaces. 
4.5 Theorem. Let $E, \tilde{E}$, and $\hat{E}$ be Hilbert spaces with group-actions $\left\{\kappa_{\varrho}\right\}$, $\left\{\tilde{\kappa}_{\varrho}\right\}$, and $\left\{\hat{\kappa}_{\varrho}\right\}$, respectively. Let $a \in M_{\gamma} S_{(c l)}^{\mu ; \ell}\left(\mathbb{R}_{+} \times \mathbb{R}^{n} \times \Gamma_{\frac{1}{2}-\gamma} ; \tilde{E}, \hat{E}\right)$ and $b \in M_{\gamma} S_{(c l)}^{\mu^{\prime} ; \ell}\left(\mathbb{R}_{+} \times \mathbb{R}^{n} \times \Gamma_{\frac{1}{2}-\gamma} ; E, \tilde{E}\right)$. Then the composition as operators on $\mathcal{T}_{\gamma}\left(\mathbb{R}_{+}, E\right)$ may be written as

$$
o p_{M}^{\gamma}(a)(\xi) \circ o p_{M}^{\gamma}(b)(\xi)=o p_{M}^{\gamma}(a \# b)(\xi)
$$

with the Leibniz-product $a \# b \in M_{\gamma} S_{(c l)}^{\mu+\mu^{\prime} ; \ell}\left(\mathbb{R}_{+} \times \mathbb{R}^{n} \times \Gamma_{\frac{1}{2}-\gamma} ; E, \hat{E}\right)$. More precisely, the Leibniz-product is given by the Mellin oscillatory integral formula

$$
a \# b(r, \xi, z)=\iint_{\mathbb{R}_{\mathbb{R}_{+}}} s^{i \eta} a(r, \xi, z+i \eta) b(r s, \xi, z) \frac{d s}{s} d \eta,
$$

and the following asymptotic expansion holds for $a \# b$ :

$$
a \# b \sim \sum_{k=0}^{\infty} \frac{1}{k !}\left(D_{\tau}^{k} a\right)\left(\left(-r \partial_{r}\right)^{k} b\right) .
$$

The mapping $(a, b) \mapsto a \# b$ is bilinear and continuous.

If $a \in M_{\gamma} S_{V(c l)}^{\mu ; \ell}\left(\mathbb{R}_{+} \times \mathbb{R}^{n} \times \mathbb{H}_{\frac{1}{2}-\gamma} ; \tilde{E}, \hat{E}\right)$ and $b \in M_{\gamma} S_{V(c l)}^{\mu^{\prime} ; \ell}\left(\mathbb{R}_{+} \times \mathbb{R}^{n} \times \mathbb{H}_{\frac{1}{2}-\gamma} ; E, \tilde{E}\right)$, then also $a \# b \in M_{\gamma} S_{V(c l)}^{\mu+\mu^{\prime} ; \ell}\left(\mathbb{R}_{+} \times \mathbb{R}^{n} \times \mathbb{H}_{\frac{1}{2}-\gamma} ; E, \hat{E}\right)$, and the oscillatory integral formula (4.ii) is valid for $z \in \mathbb{H}_{\frac{1}{2}-\gamma}$, and the asymptotic expansion (4.iii) holds in the Volterra sense, i. e.,

$$
a \# b \widetilde{V} \sum_{k=0}^{\infty} \frac{1}{k !}\left(\partial_{z}^{k} a\right)\left(\left(-r \partial_{r}\right)^{k} b\right) .
$$

In this case the mapping $(a, b) \mapsto a \# b$ is bilinear and continuous within the Volterra Mellin symbol spaces.

In the classical case we conclude from the asymptotic expansions (4.iii), (4.iv) the identity $(a \# b)_{\left(\mu+\mu^{\prime}\right)}=a_{(\mu)} b_{\left(\mu^{\prime}\right)}$ for the principal symbol of the composition.

Proof. The assertion follows from Theorem 4.3. Note that $a(r, \xi, z) b_{R}\left(r^{\prime}, \xi, z\right)$ is a double-symbol for the composition, and the Leibniz-product is the associated left-symbol. This also implies the continuity of $(a, b) \mapsto a \# b$.

The oscillatory integral formula (4.ii) necessarily holds in the preceding situation, for it holds in the non-Volterra case without parameters and trivial group-actions, and by uniqueness of analytic continuation the formula is valid within the halfplane $\mathbb{H}_{\frac{1}{2}-\gamma}$.

The asymptotic expansions (4.iii) and (4.iv) follow from (4.ii) via Taylor expansion as in the proof of Theorem 4.3. 
4.6 Proposition. Let $a\left(r, r^{\prime}, \xi, z\right) \in M_{\gamma} S^{\mu ; \ell}\left(\mathbb{R}_{+} \times \mathbb{R}_{+} \times \mathbb{R}^{n} \times \Gamma_{\frac{1}{2}-\gamma} ; E, \tilde{E}\right)$, such that $a\left(r, r^{\prime}, \xi, \lambda\right) \equiv 0$ for $\left|\frac{r}{r^{\prime}}-1\right|<\varepsilon$ for a sufficiently small $\varepsilon>0$. Then $o p_{M}^{\gamma}(a)(\xi)=o p_{M}^{\gamma}(c)(\xi)$ with a symbol $c \in M_{\gamma} S^{-\infty}\left(\mathbb{R}_{+} \times \mathbb{R}^{n} \times \Gamma_{\frac{1}{2}-\gamma} ; E, \tilde{E}\right)$.

If even $a \in M_{\gamma} S_{V}^{\mu, \ell}\left(\mathbb{R}_{+} \times \mathbb{R}_{+} \times \mathbb{R}^{n} \times \mathbb{H}_{\frac{1}{2}-\gamma} ; E, \tilde{E}\right)$, then also $c \in M_{\gamma} S_{V}^{-\infty}\left(\mathbb{R}_{+} \times\right.$ $\left.\mathbb{R}^{n} \times \mathbb{H}_{\frac{1}{2}-\gamma} ; E, \tilde{E}\right)$.

Proof. We obtain $c$ as the associated left-symbol to $a$ from Theorem 4.3. All ingredients in the asymptotic expansion of $c$ in terms of $a$ vanish by assumption which implies the assertion.

4.7 Corollary. Let $a(r, \xi, z) \in M_{\gamma} S^{\mu ; \ell}\left(\mathbb{R}_{+} \times \mathbb{R}^{n} \times \Gamma_{\frac{1}{2}-\gamma} ; E, \tilde{E}\right)$, and $\varphi, \psi \in$ $C_{B}^{\infty}\left(\mathbb{R}_{+}\right)$such that $\operatorname{dist}(\operatorname{supp} \varphi, \operatorname{supp} \psi)>0$. Then $\varphi \operatorname{op}_{M}^{\gamma}(a)(\xi) \psi=o p_{M}^{\gamma}\left(a_{\varphi, \psi}\right)(\xi)$ with a symbol $a_{\varphi, \psi} \in M_{\gamma} S^{-\infty}\left(\mathbb{R}_{+} \times \mathbb{R}^{n} \times \Gamma_{\frac{1}{2}-\gamma} ; E, \tilde{E}\right)$. The mapping $a \longmapsto a_{\varphi, \psi}$ is continuous.

If even $a(r, \xi, z) \in M_{\gamma} S_{V}^{\mu, \ell}\left(\mathbb{R}_{+} \times \mathbb{R}^{n} \times \mathbb{H}_{\frac{1}{2}-\gamma} ; E, \tilde{E}\right)$, then also $a_{\varphi, \psi} \in M_{\gamma} S_{V}^{-\infty}\left(\mathbb{R}_{+} \times\right.$ $\left.\mathbb{R}^{n} \times \mathbb{H}_{\frac{1}{2}-\gamma} ; E, \tilde{E}\right)$. In this case the mapping $a \longmapsto a_{\varphi, \psi}$ is continuous with respect to the Volterra Mellin symbol spaces.

Proof. Note that $\varphi(r) a(r, \xi, z) \psi\left(r^{\prime}\right)$ is a double-symbol associated with the operator $\varphi \mathrm{op}_{M}^{\gamma}(a)(\xi) \psi$. Consequently, we obtain $a_{\varphi, \psi}$ as the left-symbol from Theorem 4.3. From Proposition 4.6 we conclude that $a_{\varphi, \psi}$ is of order $-\infty$. The continuity of the mapping $a \longmapsto a_{\varphi, \psi}$ follows from the closed graph theorem.

4.8 Remark. From Proposition 4.6 and Corollary 4.7 it follows, in particular, that the calculus of Volterra Mellin pseudodifferential operators (with parameters) is "well-behaved" what pseudolocality is concerned, i. e., localization is possible up to remainder terms of order $-\infty$ within the Volterra calculus.

4.9 Theorem. Let $a \in M_{\gamma} S_{V}^{\mu ; \ell}\left(\mathbb{R}_{+} \times \mathbb{R}^{n} \times \mathbb{H}_{\frac{1}{2}-\gamma} ; E, \tilde{E}\right)$. Then op ${ }_{M}^{\gamma}(a)(\xi)$ restricts for every $r_{0} \in \mathbb{R}_{+}$to a family of continuous operators

$$
o p_{M}^{\gamma}(a)(\xi): \mathcal{T}_{\gamma, 0}\left(\left(0, r_{0}\right), E\right) \longrightarrow \mathcal{T}_{\gamma, 0}\left(\left(0, r_{0}\right), \tilde{E}\right) .
$$

Proof. Without loss of generality assume $n=0$. We may write

$$
\left(\operatorname{op}_{M}^{\gamma}(a) u\right)(r)=\left.\left(\mathcal{M}_{\gamma, z \rightarrow r^{\prime}}^{-1} a(r, z) \mathcal{M}_{\gamma, r^{\prime} \rightarrow z} u\right)\right|_{r^{\prime}=r}
$$

for $u \in \mathcal{T}_{\gamma}\left(\mathbb{R}_{+}, E\right)$. Now let $u \in \mathcal{T}_{\gamma, 0}\left(\left(0, r_{0}\right), E\right)$ be given and $r \in \mathbb{R}_{+}$fixed. In view of the Paley-Wiener theorem from 2.1 we have that $\mathcal{M}_{\gamma} u \in \mathcal{A}\left(\mathbb{H}_{\frac{1}{2}-\gamma}, E ; r_{0}\right)$. For $a$ is a Volterra symbol by assumption we see that $a(r, z)\left(\mathcal{M}_{\gamma} u\right)(z)$ may be regarded as an element of $\mathcal{A}\left(\mathbb{H}_{\frac{1}{2}-\gamma}, \tilde{E} ; r_{0}\right)$, i. e., $a$ acts as a "multiplier" in the 
spaces $\mathcal{A}\left(\mathbb{H}_{\frac{1}{2}-\gamma}, \cdot ; r_{0}\right)$. Employing again the Paley-Wiener theorem from 2.1 we now conclude that $\mathcal{M}_{\gamma, z \rightarrow r^{\prime}}^{-1} a(r, z) \mathcal{M}_{\gamma, r^{\prime} \rightarrow z} u \in \mathcal{T}_{\gamma, 0}\left(\left(0, r_{0}\right)_{r^{\prime}}, \tilde{E}\right)$, where the subscript $r^{\prime}$ indicates that we consider the latter function space in the variable $r^{\prime}$. In particular, evaluation at $r^{\prime}=r$ yields that (1) necessarily vanishes for $r>r_{0}$ which finishes the proof of the theorem.

4.10 Remark. Theorem 4.9 provides the motivation for the name "Volterra" symbols respectively operators:

If we regard the Mellin pseudodifferential operators as

$$
\mathrm{op}_{M}^{\gamma}(a)(\xi): C_{0}^{\infty}\left(\mathbb{R}_{+}\right) \longrightarrow C^{\infty}\left(\mathbb{R}_{+}, \mathcal{L}(E, \tilde{E})\right),
$$

then we obtain for every $r_{0} \in \mathbb{R}_{+}$that $\left(\operatorname{op}_{M}^{\gamma}(a)(\xi) u\right)(r) \equiv 0$ for $r>r_{0}$, for all functions $u \in C_{0}^{\infty}\left(\mathbb{R}_{+}\right)$such that $u \equiv 0$ for $r>r_{0}$. In other words, the operatorvalued Schwartz kernel $K_{\mathrm{op}_{M}^{\gamma}(a)(\xi)} \in \mathcal{D}^{\prime}\left(\mathbb{R}_{+} \times \mathbb{R}_{+}, \mathcal{L}(E, \tilde{E})\right)$ satisfies

$$
\operatorname{supp} K_{\mathrm{op}_{M}^{\gamma}(a)(\xi)} \subseteq\left\{\left(r, r^{\prime}\right) \in \mathbb{R}_{+} \times \mathbb{R}_{+} ; r \leq r^{\prime}\right\}
$$

for all $\xi \in \mathbb{R}^{n}$. This gives the link to (classical) Volterra integral equations where the kernel is supported on one side of the diagonal only.

4.11 Remark. A triple $\left\{E_{0}, E, E_{1} ; \kappa\right\}$ is called a Hilbert triple, if the following conditions are fulfilled:

a) There exists a Hausdorff topological vector space $X$ such that $E_{0}, E$ and $E_{1}$ are embedded in $X$.

b) $\kappa:\left(\mathbb{R}_{+}, \cdot\right) \rightarrow \mathcal{L}(X)$ is a representation, which restricts to strongly continuous group-actions on $E_{0}, E$ and $E_{1}$. On $E$ the action is assumed to be unitary.

c) $E_{0} \cap E \cap E_{1}$ is dense in $E_{0}, E$ and $E_{1}$.

d) The inner product on $E$ induces a non-degenerate sesquilinear pairing $\langle\cdot, \cdot\rangle$ : $E_{0} \times E_{1} \rightarrow \mathbb{C}$, that provides antilinear isomorphisms $E_{0}^{\prime} \cong E_{1}$ and $E_{1}^{\prime} \cong E_{0}$.

Notice, in particular, that for each $\delta \in \mathbb{R}$ the scalar product on $L^{2, \delta}\left(\mathbb{R}_{+}, E\right)$ induces a non-degenerate sesquilinear pairing

$$
\langle\cdot, \cdot\rangle: \mathcal{T}_{\delta+\gamma}\left(\mathbb{R}_{+}, E_{0}\right) \times \mathcal{T}_{\delta-\gamma}\left(\mathbb{R}_{+}, E_{1}\right) \rightarrow \mathbb{C}
$$

for every $\gamma \in \mathbb{R}$.

Let $\left\{E_{0}, E, E_{1} ; \kappa\right\}$ and $\left\{\tilde{E}_{0}, \tilde{E}, \tilde{E}_{1} ; \tilde{\kappa}\right\}$ be Hilbert triples. To each $A \in \mathcal{L}\left(E_{0}, \tilde{E}_{0}\right)$ there exists a unique (formal) adjoint operator $A^{*} \in \mathcal{L}\left(\tilde{E}_{1}, E_{1}\right)$ such that $\left\langle A e_{0}, \tilde{e}_{1}\right\rangle_{\tilde{E}}=\left\langle e_{0}, A^{*} \tilde{e}_{1}\right\rangle_{E}$ for all $e_{0} \in E_{0}$ and $\tilde{e}_{1} \in \tilde{E}_{1}$. The mapping $A \mapsto A^{*}$ provides an antilinear isomorphism $\mathcal{L}\left(E_{0}, \tilde{E}_{0}\right) \rightarrow \mathcal{L}\left(\tilde{E}_{1}, E_{1}\right)$. 
The framework of Hilbert triples provides the appropriate setting to study the behaviour of pseudodifferential operators with operator-valued symbols under taking formal adjoints, see Theorem 4.12 below. However, Volterra operators are not closed with respect to this operation, as is also obvious in view of Remark 4.10 above.

4.12 Theorem. Let $\left\{E_{0}, E, E_{1} ; \kappa\right\}$ and $\left\{\tilde{E}_{0}, \tilde{E}, \tilde{E}_{1} ; \tilde{\kappa}\right\}$ be Hilbert triples. Moreover, let $a(r, \xi, z) \in M_{\gamma} S_{(c l)}^{\mu ; \ell}\left(\mathbb{R}_{+} \times \mathbb{R}^{n} \times \Gamma_{\frac{1}{2}-\gamma} ; E_{0}, \tilde{E}_{0}\right)$, and $A(\xi):=o p_{M}^{\gamma}(a)(\xi):$ $\mathcal{T}_{\gamma}\left(\mathbb{R}_{+}, E_{0}\right) \longrightarrow \mathcal{T}_{\gamma}\left(\mathbb{R}_{+}, \tilde{E}_{0}\right)$.

For every $\delta \in \mathbb{R}$ the formal adjoint operator $A^{(*, \delta)}(\xi)$ with respect to the $L^{2, \delta}$-inner product, which is determined by the identity $\langle A(\xi) u, v\rangle_{L^{2, \delta}\left(\mathbb{R}_{+}, \tilde{E}\right)}=$ $\left\langle u, A^{(*, \delta)}(\xi) v\right\rangle_{L^{2, \delta}\left(\mathbb{R}_{+}, E\right)}$ for $u \in \mathcal{T}_{\gamma}\left(\mathbb{R}_{+}, E_{0}\right)$ and $v \in \mathcal{T}_{2 \delta-\gamma}\left(\mathbb{R}_{+}, \tilde{E}_{1}\right)$, is well-defined as a continuous operator $A^{(*, \delta)}(\xi): \mathcal{T}_{2 \delta-\gamma}\left(\mathbb{R}_{+}, \tilde{E}_{1}\right) \longrightarrow \mathcal{T}_{2 \delta-\gamma}\left(\mathbb{R}_{+}, E_{1}\right)$.

More precisely, we have $A^{(*, \delta)}(\xi)=o p_{M}^{2 \delta-\gamma}\left(a^{(*, \delta)}\right)(\xi)$ with the formal adjoint symbol $a^{(*, \delta)}(r, \xi, z) \in M_{2 \delta-\gamma} S_{(c l)}^{\mu ; \ell}\left(\mathbb{R}_{+} \times \mathbb{R}^{n} \times \Gamma_{\frac{1}{2}-2 \delta+\gamma} ; \tilde{E}_{1}, E_{1}\right)$, which is given as the left-symbol

$$
a^{(*, \delta)}(r, \xi, z)=\left(a\left(r^{\prime}, \xi, 1-2 \delta-\bar{z}\right)^{*}\right)_{L}=\int_{\mathbb{R}_{\mathbb{R}_{+}}} s^{i \eta} a(s r, \xi, 1-2 \delta-\bar{z}+i \eta)^{*} \frac{d s}{s} d \eta
$$

according to Theorem 4.3. In particular, the following asymptotic expansion of $a^{(*, \delta)}$ in terms of $a$ is valid:

$$
a^{(*, \delta)}(r, \xi, z) \sim \sum_{k=0}^{\infty} \frac{1}{k !} D_{\tau}^{k}\left(-r \partial_{r}\right)^{k} a(r, \xi, 1-2 \delta-\bar{z})^{*} .
$$

The mapping $a \mapsto a^{(*, \delta)}$ is antilinear and continuous.

In the classical case we conclude from the asymptotic expansion (4.vi) the identity $a_{(\mu)}^{(*, \delta)}=a_{(\mu)}^{*}$ for the principal symbol of the formal adjoint.

\subsection{Continuity in Mellin Sobolev spaces}

4.13 Definition. Let $E$ be a Hilbert space with group-action $\left\{\kappa_{\varrho}\right\}$. For $s, \gamma \in \mathbb{R}$ define the space $\mathcal{V}^{s, \gamma}\left(\mathbb{R}_{+}, E\right)$ to consist of all $u \in \mathcal{T}_{\gamma}^{\prime}\left(\mathbb{R}_{+}, E\right)$ such that $\mathcal{M}_{\gamma} u$ is a regular distribution in $\mathcal{S}^{\prime}\left(\Gamma_{\frac{1}{2}-\gamma}, E\right)$, and

$$
\|u\|_{\mathcal{V}^{s, \gamma}\left(\mathbb{R}_{+}, E\right)}:=\left(\frac{1}{2 \pi i} \int_{\Gamma_{\frac{1}{2}-\gamma}}\langle\operatorname{Im}(z)\rangle^{2 s}\left\|\kappa_{\langle\operatorname{Im}(z)\rangle}^{-1} \mathcal{M}_{\gamma} u(z)\right\|_{E}^{2} d z\right)^{\frac{1}{2}}<\infty
$$


If the group-action is trivial we write $\mathcal{H}^{s, \gamma}\left(\mathbb{R}_{+}, E\right)$. In case of $E=\mathbb{C}$ and trivial group-action the space is suppressed from the notation.

The following properties of these spaces are evident (see also Dorschfeldt [7], Hirschmann [17]):

i) $\mathcal{V}^{s, \gamma}\left(\mathbb{R}_{+}, E\right)$ is a Hilbert space with respect to the inner product

$$
\langle u, v\rangle_{\mathcal{V}^{s, \gamma}\left(\mathbb{R}_{+}, E\right)}:=\frac{1}{2 \pi i} \int_{\Gamma_{\frac{1}{2}-\gamma}}\langle\operatorname{Im}(z)\rangle^{2 s}\left\langle\kappa_{\langle\operatorname{Im}(z)\rangle}^{-1} \mathcal{M}_{\gamma} u(z), \kappa_{\langle\operatorname{Im}(z)\rangle}^{-1} \mathcal{M}_{\gamma} v(z)\right\rangle_{E} d z
$$

ii) The embedding $\mathcal{T}_{\gamma}\left(\mathbb{R}_{+}, E\right) \hookrightarrow \mathcal{V}^{s, \gamma}\left(\mathbb{R}_{+}, E\right)$ is continuous and dense.

iii) If $E \hookrightarrow \tilde{E}$ and the restriction of the group-action $\left\{\tilde{\kappa}_{\varrho}\right\}$ of $\tilde{E}$ on $E$ equals $\left\{\kappa_{\varrho}\right\}$, then the embedding $\mathcal{V}^{s, \gamma}\left(\mathbb{R}_{+}, E\right) \hookrightarrow \mathcal{V}^{s^{\prime}, \gamma}\left(\mathbb{R}_{+}, \tilde{E}\right)$ is well-defined and continuous for $s \geq s^{\prime}$.

iv) Let $M$ be the constant in the norm-estimate (3.iv) of the group-action from Definition 3.3. Then

$$
\mathcal{H}^{s+M, \gamma}\left(\mathbb{R}_{+}, E\right) \hookrightarrow \mathcal{V}^{s, \gamma}\left(\mathbb{R}_{+}, E\right) \hookrightarrow \mathcal{H}^{s-M, \gamma}\left(\mathbb{R}_{+}, E\right) .
$$

For $\delta \in \mathbb{R}$ the operator of multiplication with the function $r^{\delta}$ provides an isomorphism $\mathcal{V}^{s, \gamma}\left(\mathbb{R}_{+}, E\right) \longrightarrow \mathcal{V}^{s, \gamma+\delta}\left(\mathbb{R}_{+}, E\right)$.

For $r_{0} \in \mathbb{R}_{+}$we define the space $\mathcal{V}_{0}^{s, \gamma}\left(\left(0, r_{0}\right], E\right)$ to consist of all $u \in \mathcal{V}^{s, \gamma}\left(\mathbb{R}_{+}, E\right)$ such that $\operatorname{supp}(u) \subseteq\left(0, r_{0}\right]$. This is a closed subspace of $\mathcal{V}^{s, \gamma}\left(\mathbb{R}_{+}, E\right)$ and equals the closure of $\mathcal{T}_{\gamma, 0}\left(\left(0, r_{0}\right), E\right)$ in $\mathcal{V}^{s, \gamma}\left(\mathbb{R}_{+}, E\right)$.

4.14 Theorem. Let $E$ and $\tilde{E}$ be Hilbert spaces with group-actions $\left\{\kappa_{\varrho}\right\}$ and $\left\{\tilde{\kappa}_{\varrho}\right\}$, respectively. Moreover, let $M$ and $\tilde{M}$ be the constants in the norm-estimates for $\left\{\kappa_{\varrho}\right\}$ and $\left\{\tilde{\kappa}_{\varrho}\right\}$ from (3.iv). Let $a \in M_{\gamma} S^{\mu ; \ell}\left(\mathbb{R}_{+} \times \mathbb{R}^{n} \times \Gamma_{\frac{1}{2}-\gamma} ; E, \tilde{E}\right)$ and $s, \nu \in \mathbb{R}$ where $\nu \geq \frac{\mu}{\ell}$. Then $\operatorname{op}_{M}^{\gamma}(a)(\xi)$ extends for $\xi \in \mathbb{R}^{n}$ by continuity to an operator $o p_{M}^{\gamma}(a)(\xi) \in \mathcal{L}\left(\mathcal{V}^{s, \gamma}\left(\mathbb{R}_{+}, E\right), \mathcal{V}^{s-\nu, \gamma}\left(\mathbb{R}_{+}, \tilde{E}\right)\right)$, which induces a continuous embedding

$$
M_{\gamma} L^{\mu ; \ell}\left(\mathbb{R}_{+} ; \mathbb{R}^{n} ; E, \tilde{E}\right) \hookrightarrow\left\{\begin{array}{r}
S_{\perp}^{\mu+M+\tilde{M}}\left(\mathbb{R}^{n} ; \mathcal{V}^{s, \gamma}\left(\mathbb{R}_{+}, E\right), \mathcal{V}^{s-\nu, \gamma}\left(\mathbb{R}_{+}, \tilde{E}\right)\right) \\
\nu \geq 0 \\
S_{\perp}^{\mu-\nu+M+\tilde{M}}\left(\mathbb{R}^{n} ; \mathcal{V}^{s, \gamma}\left(\mathbb{R}_{+}, E\right), \mathcal{V}^{s-\nu, \gamma}\left(\mathbb{R}_{+}, \tilde{E}\right)\right) \\
\nu \leq 0
\end{array}\right.
$$

into the space of operator-valued symbols with the trivial group-action involved on the Sobolev spaces (which is indicated by the subscript 1). 
Moreover, restriction of Volterra pseudodifferential operators to the $\mathcal{V}_{0}^{s, \gamma}$-spaces provides continuous mappings

$$
M_{\gamma} L_{V}^{\mu, \ell}\left(\mathbb{R}_{+} ; \mathbb{R}^{n} ; E, \tilde{E}\right) \rightarrow\left\{\begin{array}{r}
S_{1}^{\mu+M+\tilde{M}}\left(\mathbb{R}^{n} ; \mathcal{V}_{0}^{s, \gamma}\left(\left(0, r_{0}\right], E\right), \mathcal{V}_{0}^{s-\nu, \gamma}\left(\left(0, r_{0}\right], \tilde{E}\right)\right) \\
\nu \geq 0 \\
S_{1}^{\mu-\nu+M+\tilde{M}}\left(\mathbb{R}^{n} ; \mathcal{V}_{0}^{s, \gamma}\left(\left(0, r_{0}\right], E\right), \mathcal{V}_{0}^{s-\nu, \gamma}\left(\left(0, r_{0}\right], \tilde{E}\right)\right) \\
\nu \leq 0
\end{array}\right.
$$

for each $r_{0} \in \mathbb{R}_{+}$.

Proof. The boundedness of the operators, as well as (4.vii), are easily deduced from the general continuity result for pseudodifferential operators with operatorvalued symbols given in Seiler [47], see also [23]. Thereby, the isomorphism $S_{\gamma}$ and relation (4.i) reduces the considerations to the case of Kohn-Nirenberg quantized operators in abstract edge Sobolev spaces instead of the Mellin operators, see also Schulze [40]. The second assertion concerning Volterra operators follows from (4.vii) and Theorem 4.9.

\subsection{Volterra Mellin operators with analytic symbols}

4.15 Proposition. a) Let $\emptyset \neq I \subseteq \mathbb{R}$ be an open interval. Moreover, let $a \in$ $C_{B}^{\infty}\left(\mathbb{R}_{+}, \ell_{\text {loc }}^{\infty} \mathcal{A}^{\mu ; \ell}(E, \tilde{E})\right)$ (cf. Proposition 3.25). Then for $\gamma, \gamma^{\prime} \in \mathbb{R}$ such that $\frac{1}{2}-\gamma, \frac{1}{2}-\gamma^{\prime} \in I$ we have op $p_{M}^{\gamma}(a)(\xi)=o p_{M}^{\gamma^{\prime}}(a)(\xi)$ as operators on $C_{0}^{\infty}\left(\mathbb{R}_{+}, E\right)$.

b) Let $\gamma, \delta \in \mathbb{R}$ and $a \in M_{\gamma} S^{\mu ; \ell}\left(\mathbb{R}_{+} \times \mathbb{R}^{n} \times \Gamma_{\frac{1}{2}-\gamma} ; E, \tilde{E}\right)$. Then we have $o p_{M}^{\gamma}(a)(\xi) r^{\delta}=r^{\delta} o p_{M}^{\gamma-\delta}\left(T_{-\delta} a\right)(\xi)$ as operators acting in $\mathcal{T}_{\gamma-\delta}\left(\mathbb{R}_{+}, E\right) \longrightarrow$ $\mathcal{T}_{\gamma}\left(\mathbb{R}_{+}, \tilde{E}\right)$, where $T_{-\delta} a \in M_{\gamma-\delta} S^{\mu ; \ell}\left(\mathbb{R}_{+} \times \mathbb{R}^{n} \times \Gamma_{\frac{1}{2}-\gamma+\delta} ; E, \tilde{E}\right)$ is defined as $\left(T_{-\delta} a\right)\left(r, \xi, \frac{1}{2}-\gamma+\delta+i \tau\right):=a\left(r, \xi, \frac{1}{2}-\gamma+i \tau\right)$.

Proof. For the proof of a) note that we may write for $u \in C_{0}^{\infty}\left(\mathbb{R}_{+}, E\right)$

$$
\left(\operatorname{op}_{M}^{\gamma}(a)(\xi) u\right)(r)=\frac{1}{2 \pi i} \int_{\Gamma_{\frac{1}{2}-\gamma}} r^{-\zeta} a(r, \xi, \zeta)(\mathcal{M} u)(\zeta) d \zeta .
$$

By Cauchy's theorem we may change the line of integration from $\Gamma_{\frac{1}{2}-\gamma}$ to $\Gamma_{\frac{1}{2}-\gamma^{\prime}}$ which shows a).

We have to prove the asserted identity in b) only as operators on $C_{0}^{\infty}\left(\mathbb{R}_{+}, E\right)$ in 
view of the density. We may write for $u \in C_{0}^{\infty}\left(\mathbb{R}_{+}, E\right)$

$$
\begin{aligned}
\left(\operatorname{op}_{M}^{\gamma}(a)(\xi)\left(r^{\delta} u\right)\right)(r) & =\frac{1}{2 \pi i} \int_{\Gamma_{\frac{1}{2}-\gamma}} r^{-\zeta} a(r, \xi, \zeta)(\mathcal{M} u)(\zeta+\delta) d \zeta \\
& =\frac{1}{2 \pi i} \int_{\Gamma_{\frac{1}{2}-\gamma+\delta}} r^{-(\zeta-\delta)}\left(T_{-\delta} a\right)(r, \xi, \zeta)(\mathcal{M} u)(\zeta) d \zeta \\
& =r^{\delta}\left(\operatorname{op}_{M}^{\gamma-\delta}\left(T_{-\delta} a\right)(\xi) u\right)(r) .
\end{aligned}
$$

4.16 Definition. Let $E$ and $\tilde{E}$ be Hilbert spaces endowed with strongly continuous group-actions $\left\{\kappa_{\varrho}\right\}$ and $\left\{\tilde{\kappa}_{\varrho}\right\}$, respectively. We define for $\mu \in \mathbb{R}$ the spaces of $\left(r, r^{\prime}\right)$ - resp. $r$-dependent (classical) parameter-dependent holomorphic Mellin symbols with parameter-space $\mathbb{R}^{n}$ as

$$
M S_{O(c l)}^{\mu ; \ell}\left(\left(\overline{\mathbb{R}}_{+}\right)^{q} \times \mathbb{R}^{n} \times \mathbb{C} ; E, \tilde{E}\right):=C_{B}^{\infty}\left(\left(\overline{\mathbb{R}}_{+}\right)^{q}, S_{O(c l)}^{\mu ; \ell}\left(\mathbb{R}^{n} \times \mathbb{C} ; E, \tilde{E}\right)\right)
$$

for $q=1$, 2. Correspondingly, the spaces of (classical) holomorphic Volterra Mellin symbols of order $\mu$ with parameter-space $\mathbb{R}^{n}$ are defined as

$$
M S_{V, O(c l)}^{\mu ; \ell}\left(\left(\overline{\mathbb{R}}_{+}\right)^{q} \times \mathbb{R}^{n} \times \mathbb{C} ; E, \tilde{E}\right):=C_{B}^{\infty}\left(\left(\overline{\mathbb{R}}_{+}\right)^{q}, S_{V, O(c l)}^{\mu ; \ell}\left(\mathbb{R}^{n} \times \mathbb{C} ; E, \tilde{E}\right)\right)
$$

for $q=1,2$. Analogously, we obtain the spaces of order $-\infty$. All these spaces carry Fréchet topologies in a canonical way. With the same conventions as in Definition 3.4 we also have the (Volterra) Mellin symbol spaces when we deal with scales of Hilbert spaces instead of single Hilbert spaces only.

For every $\gamma \in \mathbb{R}$ the embeddings

$$
\begin{gathered}
M S_{O(c l)}^{\mu ; \ell}\left(\left(\overline{\mathbb{R}}_{+}\right)^{q} \times \mathbb{R}^{n} \times \mathbb{C} ; E, \tilde{E}\right) \hookrightarrow M_{\gamma} S_{(c l)}^{\mu ; \ell}\left(\left(\mathbb{R}_{+}\right)^{q} \times \mathbb{R}^{n} \times \Gamma_{\frac{1}{2}-\gamma} ; E, \tilde{E}\right), \\
M S_{V, O(c l)}^{\mu ; \ell}\left(\left(\overline{\mathbb{R}}_{+}\right)^{q} \times \mathbb{R}^{n} \times \mathbb{C} ; E, \tilde{E}\right) \hookrightarrow M_{\gamma} S_{V(c l)}^{\mu ; \ell}\left(\left(\mathbb{R}_{+}\right)^{q} \times \mathbb{R}^{n} \times \mathbb{H}_{\frac{1}{2}-\gamma} ; E, \tilde{E}\right)
\end{gathered}
$$

are well-defined and continuous for $q=1,2$.

4.17 Definition. Let $\left(\mu_{k}\right) \subseteq \mathbb{R}$ be a sequence of reals such that $\mu_{k} \underset{k \rightarrow \infty}{\longrightarrow}-\infty$ and $\bar{\mu}:=\max _{k \in \mathbb{N}} \mu_{k}$. Moreover, let $a_{k} \in M S_{(V,) O}^{\mu_{k}, \ell}\left(\overline{\mathbb{R}}_{+} \times \mathbb{R}^{n} \times \mathbb{C} ; E, \tilde{E}\right)$. A symbol $a \in M S_{(V,) O}^{\bar{\mu} ; \ell}\left(\overline{\mathbb{R}}_{+} \times \mathbb{R}^{n} \times \mathbb{C} ; E, \tilde{E}\right)$ is called the asymptotic expansion of the $a_{k}$, if for every $R \in \mathbb{R}$ there is a $k_{0} \in \mathbb{N}$ such that for $k>k_{0}$

$$
a-\sum_{j=1}^{k} a_{j} \in M S_{(V,) O}^{R ; \ell}\left(\overline{\mathbb{R}}_{+} \times \mathbb{R}^{n} \times \mathbb{C} ; E, \tilde{E}\right) .
$$


The symbol $a$ is uniquely determined modulo $M S_{(V,) O}^{-\infty}\left(\overline{\mathbb{R}}_{+} \times \mathbb{R}^{n} \times \mathbb{C} ; E, \tilde{E}\right)$. We shall employ the notation $a \underset{(V)}{\sim} \sum_{j=1}^{\infty} a_{j}$.

Recall that due to Remark 3.26 this definition is not a proper refinement of the notion of asymptotic expansion of (Volterra) symbols.

4.18 Theorem. Let $\gamma \in \mathbb{R}$ and $a \in M S_{(V,) O(c l)}^{\mu ; \ell}\left(\overline{\mathbb{R}}_{+} \times \overline{\mathbb{R}}_{+} \times \mathbb{R}^{n} \times \mathbb{C} ; E, \tilde{E}\right)$. Then the Mellin left- and right-symbols $a_{L}(r, \xi, z), a_{R}\left(r^{\prime}, \xi, z\right)$ associated to the operator op $_{M}^{\gamma}(a)(\xi)$ from Theorem 4.3 belong to $M S_{(V,) O(c l)}^{\mu ; \ell}\left(\overline{\mathbb{R}}_{+} \times \mathbb{R}^{n} \times \mathbb{C} ; E, \tilde{E}\right)$ and do not depend on the particular weight $\gamma \in \mathbb{R}$. The oscillatory integral formulas for $a_{L}$ and $a_{R}$ in terms of $a$ from Theorem 4.3 hold for $z \in \mathbb{C}$, and the mappings $a \mapsto a_{L}$ and $a \mapsto a_{R}$ are continuous.

Moreover, we have the asymptotic expansions in the sense of Definition 4.17:

$$
\begin{aligned}
& \left.a_{L}(r, \xi, z) \underset{(V)}{\sim} \sum_{k=0}^{\infty} \frac{1}{k !} \partial_{z}^{k}\left(-r^{\prime} \partial_{r^{\prime}}\right)^{k} a\left(r, r^{\prime}, \xi, z\right)\right|_{r^{\prime}=r}, \\
& \left.a_{R}\left(r^{\prime}, \xi, z\right) \underset{(V)}{\sim} \sum_{k=0}^{\infty} \frac{1}{k !}(-1)^{k} \partial_{z}^{k}\left(-r \partial_{r}\right)^{k} a\left(r, r^{\prime}, \xi, z\right)\right|_{r=r^{\prime}} .
\end{aligned}
$$

Proof. We carry out the proof for the left-symbol only, because the proof for the right-symbol is analogous. In view of Theorem 4.3 the left-symbol $a_{L}$ is given by the following Mellin oscillatory integral formula:

$$
a_{L}(r, \xi, z)=\int_{\mathbb{R}_{\mathbb{R}_{+}}} s^{i \eta} a(r, s r, \xi, z+i \eta) \frac{d s}{s} d \eta
$$

which apriori holds for $z \in \Gamma_{\frac{1}{2}-\gamma}$ in the non-Volterra case, and for $z \in \mathbb{H}_{\frac{1}{2}-\gamma}$ in the Volterra case. Note that formula (1) does also make sense for $z \in \mathbb{C}$, which provides an analytic extension to the whole complex plane. More precisely, we see that $\left.a_{L}(r, \xi, z)\right|_{\Gamma_{\frac{1}{2}-\gamma}}$ is the left-symbol associated to $\left.a\left(r, r^{\prime}, \xi, z\right)\right|_{\Gamma_{\frac{1}{2}-\gamma}}$ for every $\gamma \in \mathbb{R}$, and we have

$$
a_{L}(r, \xi, z) \in \begin{cases}M_{\gamma} S_{(c l)}^{\mu ; \ell}\left(\mathbb{R}_{+} \times \mathbb{R}^{n} \times \Gamma_{\frac{1}{2}-\gamma} ; E, \tilde{E}\right) & \text { in the non-Volterra case } \\ M_{\gamma} S_{V(c l)}^{\mu ; \ell}\left(\mathbb{R}_{+} \times \mathbb{R}^{n} \times \mathbb{H}_{\frac{1}{2}-\gamma} ; E, \tilde{E}\right) & \text { in the Volterra case }\end{cases}
$$

as a smooth function of $\gamma \in \mathbb{R}$, i. e.,

$$
a_{L}(r, \xi, z) \in \begin{cases}C_{B}^{\infty}\left(\mathbb{R}_{+}, S_{O(c l)}^{\mu ; \ell}\left(\mathbb{R}^{n} \times \mathbb{C} ; E, \tilde{E}\right)\right) & \text { in the non-Volterra case } \\ C_{B}^{\infty}\left(\mathbb{R}_{+}, S_{V, O(c l)}^{\mu ; \ell}\left(\mathbb{R}^{n} \times \mathbb{C} ; E, \tilde{E}\right)\right) & \text { in the Volterra case. }\end{cases}
$$


For $\alpha \in \mathbb{N}_{0}^{n}$ and $\beta \in \mathbb{N}_{0}$ we see that $\partial_{\xi}^{\alpha} \partial_{z}^{\beta} a_{L}$ is the associated left-symbol to $\partial_{\xi}^{\alpha} \partial_{z}^{\beta} a \in M S_{(V,) O(c l)}^{\mu-|\alpha|-|\beta| \ell ; \ell}\left(\overline{\mathbb{R}}_{+} \times \overline{\mathbb{R}}_{+} \times \mathbb{R}^{n} \times \mathbb{C} ; E, \tilde{E}\right)$. Consequently, it remains to show that the derivatives with respect to $r \in \mathbb{R}_{+}$in (1) remain bounded in $S_{(V,) O(c l)}^{\mu ; \ell}\left(\mathbb{R}^{n} \times \mathbb{C} ; E, \tilde{E}\right)$ for $r \in \mathbb{R}_{+}$.

We prove by induction the following formula:

$$
\left(\partial_{r}\right)^{k} a_{L}(r, \xi, z)=\sum_{j=0}^{k}\left(\begin{array}{l}
k \\
j
\end{array}\right) \int_{\mathbb{R}_{\mathbb{R}_{+}}} s^{i \eta}\left[\left(\partial_{r}\right)^{j}\left(\partial_{r^{\prime}}\right)^{k-j} a\right](r, s r, \xi, z-(k-j)+i \eta) \frac{d s}{s} d \eta .
$$

Note that $T_{-(k-j)}\left(\partial_{r}\right)^{j}\left(\partial_{r^{\prime}}\right)^{k-j} a \in M S_{(V,) O(c l)}^{\mu ; \ell}\left(\overline{\mathbb{R}}_{+} \times \overline{\mathbb{R}}_{+} \times \mathbb{R}^{n} \times \mathbb{C} ; E, \tilde{E}\right)$ for $j \in \mathbb{N}_{0}$ in view of Proposition 3.25, where $T$ denotes the translation operator for functions in the complex plane. Consequently, formula (2) may be rewritten as

$$
\left(\partial_{r}\right)^{k} a_{L}(r, \xi, z)=\sum_{j=0}^{k}\left(\begin{array}{l}
k \\
j
\end{array}\right)\left(T_{-(k-j)}\left(\partial_{r}\right)^{j}\left(\partial_{r^{\prime}}\right)^{k-j} a\right)_{L}
$$

and from the already proven part of the theorem we obtain the boundedness of $\left(\partial_{r}\right)^{k} a_{L}$ for $r \in \mathbb{R}_{+}$as desired.

For $k=0$ formula (2) precisely becomes (1). Let us consider the case $k=1$ first. Regularizing the Mellin oscillatory integral (1) yields

$$
\begin{gathered}
a_{L}(r, \xi, z)=\iint_{\mathbb{R}_{\mathbb{R}_{+}}} s^{i \eta} a(r, s r, \xi, z+i \eta) \frac{d s}{s} d \eta=\frac{1}{2 \pi i} \int_{\Gamma_{0} \mathbb{R}_{+}} s^{\zeta}\langle\log s\rangle^{-2} \\
\left(1+\partial_{\zeta}^{2}\right)\left[\left(\frac{1}{2-\zeta^{2}}\right)^{\nu}\left(\left(2-\left(-r^{\prime} \partial_{r^{\prime}}\right)^{2}\right)^{\nu} a\right)(r, s r, \xi, z+\zeta)\right] \frac{d s}{s} d \zeta,
\end{gathered}
$$

where $\nu \in \mathbb{N}_{0}$ is chosen sufficiently large. For $s \in \mathbb{R}_{+}$we may write

$$
\begin{aligned}
& \partial_{r} \frac{1}{2 \pi i} \int_{\Gamma_{0}} s^{\zeta}\left(1+\partial_{\zeta}^{2}\right)\left[\left(\frac{1}{2-\zeta^{2}}\right)^{\nu}\left(\left(2-\left(-r^{\prime} \partial_{r^{\prime}}\right)^{2}\right)^{\nu} a\right)(r, s r, \xi, z+\zeta)\right] d \zeta \\
& =\frac{1}{2 \pi i} \int_{\Gamma_{0}} s^{\zeta}\left(1+\partial_{\zeta}^{2}\right)\left[\left(\frac{1}{2-\zeta^{2}}\right)^{\nu}\left(\left(2-\left(-r^{\prime} \partial_{r^{\prime}}\right)^{2}\right)^{\nu}\left(\partial_{r} a\right)\right)(r, s r, \xi, z+\zeta)\right] d \zeta \\
& \quad+\frac{1}{2 \pi i} \int_{\Gamma_{0}} s^{\zeta+1}\left(1+\partial_{\zeta}^{2}\right)\left[\left(\frac{1}{2-\zeta^{2}}\right)^{\nu}\left(\partial_{r^{\prime}}\left(2-\left(-r^{\prime} \partial_{r^{\prime}}\right)^{2}\right)^{\nu} a\right)(r, s r, \xi, z+\zeta)\right] d \zeta
\end{aligned}
$$


Due to Cauchy's theorem the second integral equals

$$
\begin{gathered}
\frac{1}{2 \pi i} \int_{\Gamma_{0}} s^{\zeta}\left(1+\partial_{\zeta}^{2}\right)\left[\left(\frac{1}{2-(\zeta-1)^{2}}\right)^{\nu}\left(\partial_{r^{\prime}}\left(2-\left(-r^{\prime} \partial_{r^{\prime}}\right)^{2}\right)^{\nu} a\right)(r, s r, \xi, z-1+\zeta)\right] d \zeta \\
=\frac{1}{2 \pi i} \int_{\Gamma_{0}} s^{\zeta}\left(1+\partial_{\zeta}^{2}\right)\left[\left(\frac{1}{2-(\zeta-1)^{2}}\right)^{\nu}\right. \\
\left.\left(\left(2-\left(\left(-r^{\prime} \partial_{r^{\prime}}\right)-1\right)^{2}\right)^{\nu}\left(\partial_{r^{\prime}} a\right)\right)(r, s r, \xi, z-1+\zeta)\right] d \zeta .
\end{gathered}
$$

Summing up, the derivative $\partial_{r} a_{L}(r, \xi, z)$ is given as

$$
\begin{gathered}
\frac{1}{2 \pi i} \int_{\Gamma_{0}} \int_{\mathbb{R}_{+}} s^{\zeta}\langle\log s\rangle^{-2}\left(1+\partial_{\zeta}^{2}\right)\left[\left(\frac{1}{2-\zeta^{2}}\right)^{\nu}\left(\left(2-\left(-r^{\prime} \partial_{r^{\prime}}\right)^{2}\right)^{\nu}\left(\partial_{r} a\right)\right)\right. \\
(r, s r, \xi, z+\zeta)] \frac{d s}{s} d \zeta+\frac{1}{2 \pi i} \iint_{\Gamma_{0} \mathbb{R}_{+}} s^{\zeta}\langle\log s\rangle^{-2}\left(1+\partial_{\zeta}^{2}\right)\left[\left(\frac{1}{2-(\zeta-1)^{2}}\right)^{\nu}\right. \\
\left.\left(\left(2-\left(\left(-r^{\prime} \partial_{r^{\prime}}\right)-1\right)^{2}\right)^{\nu}\left(\partial_{r^{\prime}} a\right)\right)(r, s r, \xi, z-1+\zeta)\right] \frac{d s}{s} d \zeta= \\
=\frac{1}{2 \pi i} \int_{\Gamma_{0}} \int_{\mathbb{R}_{+}} s^{\zeta}\left(\partial_{r} a\right)(r, s r, \xi, z+\zeta) \frac{d s}{s} d \zeta+ \\
+\frac{1}{2 \pi i} \int_{\Gamma_{0}} \int_{\mathbb{R}_{+}} s^{\zeta}\left(\partial_{r^{\prime}} a\right)(r, s r, \xi, z-1+\zeta) \frac{d s}{s} d \zeta
\end{gathered}
$$

i. e., formula (2) holds for $k=1$. Now assume that (2), (3) holds for some $k \in \mathbb{N}_{0}$. We may apply the above considerations involving one derivative only to the terms in the summation in (3). This at once implies the validity of (2), (3) in case of $k+1$ derivatives, i. e., these formulas hold for all $k \in \mathbb{N}_{0}$ by induction. The continuity of the mappings $a \mapsto a_{L}$ and $a \mapsto a_{R}$ follow from the closed graph theorem.

The asymptotic expansion formulas for $a_{L}$ and $a_{R}$ in terms of $a$ follow with the above considerations analogously to the proof of Theorem 4.3 via Taylor expansion in the Mellin oscillatory integral formulas.

\subsection{Definition. Define}

$$
\begin{aligned}
M_{O} L_{(c l)}^{\mu ; \ell}\left(\mathbb{R}_{+} ; \mathbb{R}^{n} ; E, \tilde{E}\right) & :=\left\{\operatorname{op}_{M}^{\gamma}(a)(\xi) ; a \in M S_{O(c l)}^{\mu ; \ell}\left(\overline{\mathbb{R}}_{+} \times \mathbb{R}^{n} \times \mathbb{C} ; E, \tilde{E}\right)\right\} \\
M_{V, O} L_{(c l)}^{\mu ; \ell}\left(\mathbb{R}_{+} ; \mathbb{R}^{n} ; E, \tilde{E}\right) & :=\left\{\operatorname{op}_{M}^{\gamma}(a)(\xi) ; a \in M S_{V, O(c l)}^{\mu ; \ell}\left(\overline{\mathbb{R}}_{+} \times \mathbb{R}^{n} \times \mathbb{C} ; E, \tilde{E}\right)\right\}
\end{aligned}
$$

In view of Theorem $4.18 \mathrm{op}_{M}^{\gamma}(\cdot)(\xi)$ provides an isomorphism between the operator spaces and the corresponding (left-) symbol spaces. Via that isomorphism we carry over the topologies which turns the operator spaces into Fréchet spaces. 
We do not refer to the particular weight $\gamma \in \mathbb{R}$ which is on the one hand justified by Theorem 4.18, and on the other hand by Proposition 4.15.

4.20 Theorem. Let $E, \tilde{E}$, and $\hat{E}$ be Hilbert spaces with group-actions $\left\{\kappa_{\ell}\right\}$, $\left\{\tilde{\kappa}_{\varrho}\right\}$, and $\left\{\hat{\kappa}_{\varrho}\right\}$, respectively. Let $a \in M S_{(V,) O(c l)}^{\mu ; \ell}\left(\overline{\mathbb{R}}_{+} \times \mathbb{R}^{n} \times \mathbb{C} ; \tilde{E}, \hat{E}\right)$ and $b \in M S_{(V,) O(c l)}^{\mu^{\prime} ; \ell}\left(\overline{\mathbb{R}}_{+} \times \mathbb{R}^{n} \times \mathbb{C} ; E, \tilde{E}\right)$. Then the Leibniz-product $a \# b$ belongs to $M S_{(V,) O(c l)}^{\mu+\mu^{\prime} ; \ell}\left(\overline{\mathbb{R}}_{+} \times \mathbb{R}^{n} \times \mathbb{C} ; E, \hat{E}\right)$ and is independent of the particular weight $\gamma \in \mathbb{R}$. The oscillatory integral formula (4.ii) for $a \# b$ in terms of $a$ and $b$ from Theorem 4.5 holds for $z \in \mathbb{C}$, and the mapping $(a, b) \mapsto a \# b$ is bilinear and continuous.

The following asymptotic expansion holds for $a \# b$ in the sense of Definition 4.17:

$$
a \# b \underset{(V)}{\sim} \sum_{k=0}^{\infty} \frac{1}{k !}\left(\partial_{z}^{k} a\right)\left(\left(-r \partial_{r}\right)^{k} b\right) .
$$

Moreover, we have the following formula for the derivatives of the Leibniz-product:

$$
\partial_{r}^{k}(a \# b)=\sum_{j=0}^{k}\left(\begin{array}{l}
k \\
j
\end{array}\right)\left(T_{-(k-j)} \partial_{r}^{j} a\right) \#\left(\partial_{r}^{k-j} b\right)
$$

where $T$ denotes the translation operator for functions in the complex plane.

Proof. The assertions follow analogously to the proof of Theorem 4.5 from Theorem 4.18. Formula (4.ix) for the higher derivatives of the Leibniz-product follows as in the proof of formula (2), (3) in the proof of Theorem 4.18 via investigating the oscillatory integral formula for the Leibniz-product (4.ii).

4.21 Definition. Let op $M_{M}^{\gamma}(a)(\xi) \in M_{O} L^{\mu, \ell}\left(\mathbb{R}_{+} ; \mathbb{R}^{n} ; \tilde{E}, \hat{E}\right)$. For $k \in \mathbb{N}_{0}$ we define the conormal symbol of order $-k$ via

$$
\sigma_{M}^{-k}\left(\operatorname{op}_{M}^{\gamma}(a)(\xi)\right)(\xi, z):=\frac{1}{k !}\left(\partial_{r}^{k} a\right)(0, \xi, z) .
$$

The conormal symbol of order 0 is also called conormal symbol simply.

Let $\operatorname{op}_{M}^{\gamma}(b)(\xi) \in M_{O} L^{\mu^{\prime} ; \ell}\left(\mathbb{R}_{+} ; \mathbb{R}^{n} ; E, \tilde{E}\right)$. Then we obtain from (4.ix) the following formula for the conormal symbols of the composition

$$
\sigma_{M}^{-k}\left(\mathrm{op}_{M}^{\gamma}(a \# b)(\xi)\right)=\sum_{p+q=k} T_{-q} \sigma_{M}^{-p}\left(\mathrm{op}_{M}^{\gamma}(a)(\xi)\right) \sigma_{M}^{-q}\left(\mathrm{op}_{M}^{\gamma}(b)(\xi)\right),
$$

where $T$ denotes the translation operator for functions in the complex plane.

4.22 Proposition. Let $a\left(r, r^{\prime}, \xi, z\right) \in M S_{(V,) O}^{\mu ; \ell}\left(\overline{\mathbb{R}}_{+} \times \overline{\mathbb{R}}_{+} \times \mathbb{R}^{n} \times \mathbb{C} ; E, \tilde{E}\right)$, such that $a\left(r, r^{\prime}, \xi, \lambda\right) \equiv 0$ for $\left|\frac{r}{r^{\prime}}-1\right|<\varepsilon$ for a sufficiently small $\varepsilon>0$. Then $o p_{M}^{\gamma}(a)(\xi)=$ $o p_{M}^{\gamma}(c)(\xi)$ with a symbol $c \in M S_{(V,) O}^{-\infty}\left(\overline{\mathbb{R}}_{+} \times \mathbb{R}^{n} \times \mathbb{C} ; E, \tilde{E}\right)$. 
4.23 Corollary. Let $a(r, \xi, z) \in M S_{(V,) O}^{\mu, \ell}\left(\overline{\mathbb{R}}_{+} \times \mathbb{R}^{n} \times \mathbb{C} ; E, \tilde{E}\right)$, and $\varphi, \psi \in C_{B}^{\infty}\left(\overline{\mathbb{R}}_{+}\right)$ such that $\operatorname{dist}(\operatorname{supp} \varphi, \operatorname{supp} \psi)>0$. Then $\varphi \operatorname{op}_{M}^{\gamma}(a)(\xi) \psi=o p_{M}^{\gamma}\left(a_{\varphi, \psi}\right)(\xi)$ with a symbol $a_{\varphi, \psi} \in M S_{(V,) O}^{-\infty}\left(\overline{\mathbb{R}}_{+} \times \mathbb{R}^{n} \times \mathbb{C} ; E, \tilde{E}\right)$. The mapping $a \longmapsto a_{\varphi, \psi}$ is continuous.

4.24 Theorem. Let $\left\{E_{0}, E, E_{1} ; \kappa\right\}$ and $\left\{\tilde{E}_{0}, \tilde{E}, \tilde{E}_{1} ; \tilde{\kappa}\right\}$ be Hilbert triples, and let $a(r, \xi, z) \in M S_{O(c l)}^{\mu ; \ell}\left(\overline{\mathbb{R}}_{+} \times \mathbb{R}^{n} \times \mathbb{C} ; E_{0}, \tilde{E}_{0}\right)$.

For each $\delta \in \mathbb{R}$ the formal adjoint symbol $a^{(*, \delta)}(r, \xi, z)$ from Theorem 4.12 belongs to $M S_{O(c l)}^{\mu ; \ell}\left(\overline{\mathbb{R}}_{+} \times \mathbb{R}^{n} \times \mathbb{C} ; \tilde{E}_{1}, E_{1}\right)$, and it is independent of the particular weight $\gamma \in \mathbb{R}$. The identity (4.v) is valid for all $z \in \mathbb{C}$, and the mapping $a \mapsto a^{(*, \delta)}$ is antilinear and continuous.

Moreover, the following asymptotic expansion of $a^{(*, \delta)}$ in terms of $a$ holds in the sense of Definition 4.17:

$$
a^{(*, \delta)}(r, \xi, z) \sim \sum_{k=0}^{\infty} \frac{1}{k !} \partial_{z}^{k}\left(-r \partial_{r}\right)^{k} a(r, \xi, 1-2 \delta-\bar{z})^{*} .
$$

\section{Kernel cut-off and Mellin quantization}

\subsection{The Mellin kernel cut-off operator}

5.1 Definition. Let $E$ and $\tilde{E}$ be Hilbert spaces with group-actions $\left\{\kappa_{\varrho}\right\}$ and $\left\{\tilde{\kappa}_{\varrho}\right\}$, respectively. Define the Mellin kernel cut-off operator with respect to the weight $\gamma \in \mathbb{R}$ by means of the Mellin oscillatory integral

$$
\left(H_{\gamma}(\varphi) a\right)(\xi, z):=\int_{\mathbb{R}} \int_{0}^{\infty} r^{i \tau} \varphi(r) a(\xi, z-i \tau) \frac{d r}{r} d \tau
$$

for $(\xi, z) \in \mathbb{R}^{n} \times \Gamma_{\frac{1}{2}-\gamma}$ and $\varphi \in C_{B}^{\infty}\left(\mathbb{R}_{+}\right), a \in S^{\mu ; \ell}\left(\mathbb{R}^{n} \times \Gamma_{\frac{1}{2}-\gamma} ; E, \tilde{E}\right)$. Analogous notions apply in case of scales of Hilbert spaces involved.

5.2 Remark. We may rewrite (5.i) as

$$
\left(H_{\gamma}(\varphi) a\right)\left(\xi, \frac{1}{2}-\gamma+i \tau\right)=\left(H\left(S_{\frac{1}{2}} \varphi\right) a_{\gamma}\right)(\xi, \tau)
$$

with the Fourier kernel cut-off operator

$$
(H(\psi) b)(\xi, \tau):=\int_{\mathbb{R}} \int_{\mathbb{R}} e^{-i t \lambda} \psi(t) b(\xi, \tau-\lambda) d t d \lambda,
$$


where $S_{\frac{1}{2}}: C_{B}^{\infty}\left(\mathbb{R}_{+}\right) \longrightarrow C_{b}^{\infty}(\mathbb{R})$ is as in $(2 . \mathrm{i})$, and $a_{\gamma}(\xi, \tau):=a\left(\xi, \frac{1}{2}-\gamma+i \tau\right)$. Therefore, the properties of the Mellin kernel cut-off operator can be traced back to those of the Fourier kernel cut-off operator, which is rigorously discussed, e. g., in Krainer [23], [24]. In particular, the following theorem that summarizes some of the relevant functional analytic properties is a consequence of this relation.

5.3 Theorem. Let $\left\{E_{j}\right\}$ and $\left\{\tilde{E}_{j}\right\}$ be scales of Hilbert spaces with group-actions $\left\{\kappa_{\varrho}\right\}$ and $\left\{\tilde{\kappa}_{\varrho}\right\}$, respectively, as in Definition 3.4. We again use the abbreviations $\mathcal{E}$ and $\tilde{\mathcal{E}}$ from Notation 3.5 .

The Mellin kernel cut-off operator with respect to the weight $\gamma \in \mathbb{R}$ is a continuous bilinear mapping in the spaces

$$
H_{\gamma}: C_{B}^{\infty}\left(\mathbb{R}_{+}\right) \times S_{(c l)}^{\mu ; \ell}\left(\mathbb{R}^{n} \times \Gamma_{\frac{1}{2}-\gamma} ; \mathcal{E}, \tilde{\mathcal{E}}\right) \longrightarrow S_{(c l)}^{\mu ; \ell}\left(\mathbb{R}^{n} \times \Gamma_{\frac{1}{2}-\gamma} ; \mathcal{E}, \tilde{\mathcal{E}}\right) .
$$

It restricts to continuous bilinear mappings in the spaces

$$
H_{\gamma}:\left\{\begin{array}{l}
C_{B}^{\infty}\left(\mathbb{R}_{+}\right) \times S_{V(c l)}^{\mu ; \ell}\left(\mathbb{R}^{n} \times \mathbb{H}_{\frac{1}{2}-\gamma} ; \mathcal{E}, \tilde{\mathcal{E}}\right) \longrightarrow S_{V(c l)}^{\mu ; \ell}\left(\mathbb{R}^{n} \times \mathbb{H}_{\frac{1}{2}-\gamma} ; \mathcal{E}, \tilde{\mathcal{E}}\right) \\
C_{0}^{\infty}\left(\mathbb{R}_{+}\right) \times S_{(c l)}^{\mu ; \ell}\left(\mathbb{R}^{n} \times \Gamma_{\frac{1}{2}-\gamma} ; \mathcal{E}, \tilde{\mathcal{E}}\right) \longrightarrow S_{O(c l)}^{\mu ; \ell}\left(\mathbb{R}^{n} \times \mathbb{C} ; \mathcal{E}, \tilde{\mathcal{E}}\right) \\
C_{0}^{\infty}\left(\mathbb{R}_{+}\right) \times S_{V(c l)}^{\mu ; \ell}\left(\mathbb{R}^{n} \times \mathbb{H}_{\frac{1}{2}-\gamma} ; \mathcal{E}, \tilde{\mathcal{E}}\right) \longrightarrow S_{V, O(c l)}^{\mu ; \ell}\left(\mathbb{R}^{n} \times \mathbb{C} ; \mathcal{E}, \tilde{\mathcal{E}}\right)
\end{array}\right.
$$

The following asymptotic expansion holds for $H_{\gamma}(\varphi) a \in S^{\mu ; \ell}\left(\mathbb{R}^{n} \times \Gamma_{\frac{1}{2}-\gamma} ; \mathcal{E}, \tilde{\mathcal{E}}\right)$ in terms of $\varphi \in C_{B}^{\infty}\left(\mathbb{R}_{+}\right)$and $a \in S^{\mu ; \ell}\left(\mathbb{R}^{n} \times \Gamma_{\frac{1}{2}-\gamma} ; \mathcal{E}, \tilde{\mathcal{E}}\right)$ :

$$
\left.H_{\gamma}(\varphi) a \sim \sum_{k=0}^{\infty} \frac{1}{k !}\left(r \partial_{r}\right)^{k} \varphi(r)\right|_{r=1} \cdot D_{\tau}^{k} a
$$

In case of Volterra symbols we obtain

$$
\left.H_{\gamma}(\varphi) a \underset{V}{\sim} \sum_{k=0}^{\infty} \frac{1}{k !}\left(r \partial_{r}\right)^{k} \varphi(r)\right|_{r=1} \cdot \partial_{z}^{k} a
$$

If $\varphi \in C_{0}^{\infty}\left(\mathbb{R}_{+}\right)$and $a \in S^{\mu ; \ell}\left(\mathbb{R}^{n} \times \Gamma_{\frac{1}{2}-\gamma} ; \mathcal{E}, \tilde{\mathcal{E}}\right)$ we have for every $\delta \in \mathbb{R}$ the following asymptotic expansion of $\left.H_{\gamma}(\varphi) a\right|_{\Gamma_{\frac{1}{2}-\gamma-\delta}} \in S^{\mu ; \ell}\left(\mathbb{R}^{n} \times \Gamma_{\frac{1}{2}-\gamma-\delta} ; \mathcal{E}, \tilde{\mathcal{E}}\right)$ in terms of $\varphi$ and $a$ :

$$
\left.\left.H_{\gamma}(\varphi) a\right|_{\Gamma_{\frac{1}{2}-\gamma-\delta}} \sim \sum_{k=0}^{\infty} \frac{1}{k !}\left(r \partial_{r}\right)^{k} r^{-\delta} \varphi(r)\right|_{r=1} \cdot D_{\tau}^{k} a
$$

If $\psi \in C_{0}^{\infty}\left(\mathbb{R}_{+}\right)$such that $\psi \equiv 1$ near $r=1$, then the operator $I-H_{\gamma}(\psi)$ is continuous in the spaces

$$
I-H_{\gamma}(\psi):\left\{\begin{array}{l}
S^{\mu ; \ell}\left(\mathbb{R}^{n} \times \Gamma_{\frac{1}{2}-\gamma} ; \mathcal{E}, \tilde{\mathcal{E}}\right) \longrightarrow S^{-\infty}\left(\mathbb{R}^{n} \times \Gamma_{\frac{1}{2}-\gamma} ; \mathcal{E}, \tilde{\mathcal{E}}\right) \\
S_{V}^{\mu ; \ell}\left(\mathbb{R}^{n} \times \mathbb{H}_{\frac{1}{2}-\gamma} ; \mathcal{E}, \tilde{\mathcal{E}}\right) \longrightarrow S_{V}^{-\infty}\left(\mathbb{R}^{n} \times \mathbb{H}_{\frac{1}{2}-\gamma} ; \mathcal{E}, \tilde{\mathcal{E}}\right)
\end{array}\right.
$$


5.4 Remark. The Mellin kernel cut-off operator can be used to give a proof for the existence of Volterra symbols having a prescribed asymptotic expansion.

More precisely, let $a_{j} \in S_{V}^{\mu_{j} ; \ell}\left(\mathbb{R}^{n} \times \mathbb{H}_{\frac{1}{2}-\gamma} ; \mathcal{E}, \tilde{\mathcal{E}}\right)$, where $\mathcal{E}$ and $\tilde{\mathcal{E}}$ are as in Notation 3.5 , and $\mu_{j} \rightarrow-\infty$ as $j \rightarrow \infty$. To construct a symbol $a \in S_{V}^{\bar{\mu} ; \ell}\left(\mathbb{R}^{n} \times \mathbb{H}_{\frac{1}{2}-\gamma} ; \mathcal{E}, \tilde{\mathcal{E}}\right)$, where $\bar{\mu}=\max \left\{\mu_{j} ; j \in \mathbb{N}\right\}$, with $a \underset{V}{\sim} \sum_{j=1}^{\infty} a_{j}$, we may define the following unconditionally convergent sum

$$
a:=\sum_{j=1}^{\infty} H_{\gamma}\left(\psi\left(r^{c_{j}}\right)\right) a_{j}
$$

where $\psi \in C_{0}^{\infty}\left(\mathbb{R}_{+}\right)$with $\psi \equiv 1$ near $r=1$, and $\left(c_{j}\right)_{j \in \mathbb{N}} \subseteq \mathbb{R}_{+}$with $c_{j} \rightarrow \infty$ as $j \rightarrow \infty$ sufficiently fast. To establish the convergence of this series makes use of the analyticity of Volterra symbols and a related Paley-Wiener effect in the Mellin preimage. Once this is achieved the desired assertion follows, using Theorem 5.3. Moreover, by replacing $a$ by $H_{\gamma}(\psi) a$, we can even construct $a$ as a holomorphic Volterra Mellin symbol, which provides a proof for the existence of holomorphic Volterra Mellin symbols with a prescribed asymptotic expansion.

A rigorous discussion based on the Fourier kernel cut-off operator can be found in [23], [24] (see also Remark 5.2 above).

\subsection{Degenerate symbols and Mellin quantization}

5.5 Lemma. Define $M: \mathbb{R}_{+} \times \mathbb{R}_{+} \rightarrow \mathbb{R}$ via

$$
M\left(r, r^{\prime}\right):= \begin{cases}\frac{\log (r)-\log \left(r^{\prime}\right)}{r-r^{\prime}} & \text { if } r \neq r^{\prime} \\ \frac{1}{r} & \text { if } r=r^{\prime} .\end{cases}
$$

Then $M \in C^{\infty}\left(\mathbb{R}_{+} \times \mathbb{R}_{+}\right)$, and $M$ is strictly positive.

Proof. That $M$ is strictly positive is evident from the definition. For $r \neq r^{\prime}$ such that $\left|r-r^{\prime}\right|<r^{\prime}$ we may write

$$
M\left(r, r^{\prime}\right)=\frac{1}{r-r^{\prime}} \log \left(1+\frac{r-r^{\prime}}{r^{\prime}}\right)=\sum_{k=0}^{\infty} \frac{(-1)^{k}}{k+1} \frac{\left(r-r^{\prime}\right)^{k}}{r^{\prime k+1}} .
$$

This shows that $M$ is a smooth function as asserted.

5.6 Definition. Let $E$ and $\tilde{E}$ be Hilbert spaces with group-actions $\left\{\kappa_{\varrho}\right\}$ and $\left\{\tilde{\kappa}_{\varrho}\right\}$, respectively. For $\varphi \in C_{0}^{\infty}\left(\mathbb{R}_{+}\right)$and $a \in S^{\mu ; \ell}\left(\mathbb{R}^{n} \times \mathbb{R} ; E, \tilde{E}\right)$ define

$$
Q(\varphi, a)(\xi, z):=\int_{\mathbb{R}} \int_{\mathbb{R}} e^{-i s \eta} e^{i \eta} s^{-z} \varphi(s) a(\xi, \eta) d s d \eta
$$


for $(\xi, z) \in \mathbb{R}^{n} \times \mathbb{C}$.

Moreover, for every $\gamma \in \mathbb{R}$ we define for $\psi \in C_{0}^{\infty}\left(\mathbb{R}_{+}\right)$and $a \in S^{\mu ; \ell}\left(\mathbb{R}^{n} \times\right.$ $\left.\Gamma_{\frac{1}{2}-\gamma} ; E, \tilde{E}\right)$

$$
\tilde{Q}_{\gamma}(\psi, a)(\xi, z):=\frac{1}{2 \pi i} \int_{\Gamma_{\frac{1}{2}-\gamma}} \int_{0}^{\infty} s^{\zeta} e^{i(s-1) z} \psi(s) a(\xi, \zeta) \frac{d s}{s} d \zeta
$$

for $(\xi, z) \in \mathbb{R}^{n} \times \mathbb{C}$.

If $\varphi \equiv 1$ near $r=1$, and $\psi \equiv 1$ near $r=1$, we simply write $Q(\varphi, a)=Q(a)$ and $\tilde{Q}_{\gamma}(\psi, a)=\tilde{Q}_{\gamma}(a)$, respectively. The mapping $Q$ is called Mellin quantization, $\tilde{Q}_{\gamma}$ is called inverse Mellin quantization with respect to the weight $\gamma \in \mathbb{R}$.

5.7 Theorem. Let $\left\{E_{j}\right\}$ and $\left\{\tilde{E}_{j}\right\}$ be scales of Hilbert spaces with group-actions $\left\{\kappa_{\varrho}\right\}$ and $\left\{\tilde{\kappa}_{\varrho}\right\}$, respectively, and $\mathcal{E}$ and $\tilde{\mathcal{E}}$ as in Notation 3.5.

a) The operator $Q$ from (5.ii) provides continuous bilinear mappings

$$
Q:\left\{\begin{array}{l}
C_{0}^{\infty}\left(\mathbb{R}_{+}\right) \times S_{(c l)}^{\mu ; \ell}\left(\mathbb{R}^{n} \times \mathbb{R} ; \mathcal{E}, \tilde{\mathcal{E}}\right) \longrightarrow S_{O(c l)}^{\mu ; \ell}\left(\mathbb{R}^{n} \times \mathbb{C} ; \mathcal{E}, \tilde{\mathcal{E}}\right) \\
C_{0}^{\infty}\left(\mathbb{R}_{+}\right) \times S_{V(c l)}^{\mu ; \ell}\left(\mathbb{R}^{n} \times \mathbb{H} ; \mathcal{E}, \tilde{\mathcal{E}}\right) \longrightarrow S_{V, O(c l)}^{\mu ; \ell}\left(\mathbb{R}^{n} \times \mathbb{C} ; \mathcal{E}, \tilde{\mathcal{E}}\right)
\end{array}\right.
$$

Moreover, there are universal coefficients $\left(c_{k, j}(\varphi, \gamma)\right)$ depending neither on a nor on the Hilbert spaces, but only on $\left\{\left(\partial_{r}^{\nu} \varphi\right)(1) ; \nu \in \mathbb{N}_{0}\right\}$ and $\gamma \in \mathbb{R}$, such that the following asymptotic expansion holds for $\left.Q(\varphi, a)\right|_{\Gamma_{\frac{1}{2}-\gamma}}$, respectively $\left.Q(\varphi, a)\right|_{\mathbb{H}_{\frac{1}{2}-\gamma}}$, in terms of $a$ :

$Q(\varphi, a)\left(\xi, \frac{1}{2}-\gamma+i \tau\right) \underset{(V)}{\sim} \varphi(1) a(\xi,-\tau)+\sum_{k=1}^{\infty} \sum_{j=0}^{k} c_{k, j}(\varphi, \gamma)(-\tau)^{j}\left(\partial_{\tau}^{k+j} a\right)(\xi,-\tau)$

for $\tau \in \mathbb{R}$, respectively $\tau \in \mathbb{H}_{-}$.

b) The operator $\tilde{Q}_{\gamma}$ from (5.iii) provides continuous bilinear mappings

$$
\tilde{Q}_{\gamma}:\left\{\begin{array}{l}
C_{0}^{\infty}\left(\mathbb{R}_{+}\right) \times S_{(c l)}^{\mu ; \ell}\left(\mathbb{R}^{n} \times \Gamma_{\frac{1}{2}-\gamma} ; \mathcal{E}, \tilde{\mathcal{E}}\right) \longrightarrow S_{i O(c l)}^{\mu ; \ell}\left(\mathbb{R}^{n} \times \mathbb{C} ; \mathcal{E}, \tilde{\mathcal{E}}\right) \\
C_{0}^{\infty}\left(\mathbb{R}_{+}\right) \times S_{V(c l)}^{\mu ; \ell}\left(\mathbb{R}^{n} \times \mathbb{H}_{\frac{1}{2}-\gamma} ; \mathcal{E}, \tilde{\mathcal{E}}\right) \longrightarrow S_{V, i O(c l)}^{\mu ; \ell}\left(\mathbb{R}^{n} \times \mathbb{C} ; \mathcal{E}, \tilde{\mathcal{E}}\right)
\end{array}\right.
$$

The spaces in the image are given by means of the isomorphism

$$
S_{(V,) i O(c l)}^{\mu ; \ell}\left(\mathbb{R}^{n} \times \mathbb{C} ; \mathcal{E}, \tilde{\mathcal{E}}\right) \ni a(\xi, z) \longmapsto a(\xi,-i z) \in S_{(V,) O(c l)}^{\mu ; \ell}\left(\mathbb{R}^{n} \times \mathbb{C} ; \mathcal{E}, \tilde{\mathcal{E}}\right) .
$$

Moreover, there are universal coefficients $\left(d_{k, j}(\psi, \gamma)\right)$ depending neither on a nor on the Hilbert spaces, but only on $\left\{\left(\partial_{r}^{\nu} \psi\right)(1) ; \nu \in \mathbb{N}_{0}\right\}$ and $\gamma \in \mathbb{R}$, 
such that the following asymptotic expansion holds for $\left.\tilde{Q}_{\gamma}(\psi, a)\right|_{\mathbb{R}}$, respectively $\left.\tilde{Q}_{\gamma}(\psi, a)\right|_{\mathbb{H}}$, in terms of $a$ :

$$
\begin{aligned}
\tilde{Q}_{\gamma}(\psi, a)(\xi, \tau) \underset{(V)}{\sim} \psi(1) & a\left(\xi, \frac{1}{2}-\gamma-i \tau\right) \\
& +\sum_{k=1}^{\infty} \sum_{j=0}^{k} d_{k, j}(\psi, \gamma)(-i \tau)^{j}\left(\partial_{\tau}^{k+j} a\right)\left(\xi, \frac{1}{2}-\gamma-i \tau\right)
\end{aligned}
$$

for $\tau \in \mathbb{R}$, respectively $\tau \in \mathbb{H}$.

c) For $\varphi, \psi \in C_{0}^{\infty}\left(\mathbb{R}_{+}\right)$such that $\varphi \equiv 1$ and $\psi \equiv 1$ near $r=1$ we have

$$
\begin{aligned}
& Q\left(\tilde{Q}_{\gamma}(a)\right)-a \in\left\{\begin{array}{l}
S^{-\infty}\left(\mathbb{R}^{n} \times \Gamma_{\frac{1}{2}-\gamma} ; \mathcal{E}, \tilde{\mathcal{E}}\right) \\
S_{V}^{-\infty}\left(\mathbb{R}^{n} \times \mathbb{H}_{\frac{1}{2}-\gamma} ; \mathcal{E}, \tilde{\mathcal{E}}\right),
\end{array}\right. \\
& \tilde{Q}_{\gamma}(Q(a))-a \in\left\{\begin{array}{l}
S^{-\infty}\left(\mathbb{R}^{n} \times \mathbb{R} ; \mathcal{E}, \tilde{\mathcal{E}}\right) \\
S_{V}^{-\infty}\left(\mathbb{R}^{n} \times \mathbb{H} ; \mathcal{E}, \tilde{\mathcal{E}}\right) .
\end{array}\right.
\end{aligned}
$$

Proof. We may restrict ourselves to the case of single Hilbert spaces $E$ and $\tilde{E}$. For the proof of the continuity of $Q$ and $\tilde{Q}_{\gamma}$ in a) and b) we simply have to show that the corresponding spaces are mapped into each other as asserted. The (separate) continuity then follows from the closed graph theorem. From the asymptotic expansions in a) and b) we obtain that classical symbols are mapped to classical symbols, hence it suffices to consider general symbols and to show the validity of the expansions (see also Proposition 3.25). Let us first prove a). Clearly, $Q(\varphi, a)(\xi, z)$ depends holomorphically on $z \in \mathbb{C}$. Moreover, we may write

$$
Q(\varphi, a)(\xi, \delta+i \tau)=Q\left(r^{-\delta} \varphi(r), a\right)(\xi, i \tau) .
$$

For the family $\left\{r^{-\delta} \varphi(r)\right\} \subseteq C_{0}^{\infty}\left(\mathbb{R}_{+}\right)$is locally uniformly bounded for $\delta \in \mathbb{R}$, the proof reduces to show, using Proposition 3.25, that

$$
Q:\left\{\begin{array}{l}
C_{0}^{\infty}\left(\mathbb{R}_{+}\right) \times S^{\mu ; \ell}\left(\mathbb{R}^{n} \times \mathbb{R} ; E, \tilde{E}\right) \longrightarrow S^{\mu ; \ell}\left(\mathbb{R}^{n} \times \Gamma_{0} ; E, \tilde{E}\right) \\
C_{0}^{\infty}\left(\mathbb{R}_{+}\right) \times S_{V}^{\mu ; \ell}\left(\mathbb{R}^{n} \times \mathbb{H} ; E, \tilde{E}\right) \longrightarrow S_{V}^{\mu ; \ell}\left(\mathbb{R}^{n} \times \mathbb{H}_{0} ; E, \tilde{E}\right)
\end{array}\right.
$$

as continuous bilinear mappings, i. e., the spaces are mapped into each other, as well as the asymptotic expansion. With the function $M$ from Lemma 5.5 we may rewrite $Q(\varphi, a)$ as

$$
Q(\varphi, a)(\xi, i \tau)=\int_{\mathbb{R}} \int_{0}^{\infty} s^{i \eta} \varphi(s) s M(s, 1) a(\xi,-M(s, 1)(\tau+\eta)) \frac{d s}{s} d \eta
$$


for either $\tau \in \mathbb{R}$ or $\tau \in \mathbb{H}$ _ in the Volterra case. Note that $\varphi$ has compact support in $\mathbb{R}_{+}$. Regularizing the oscillatory integral reveals that $Q(\varphi, a)$ belongs to the corresponding spaces as asserted.

Let us now prove the asymptotic expansion. Carrying out a Taylor expansion in $\eta=0$ shows that we may write for each $N \in \mathbb{N}$ :

$$
\begin{aligned}
Q(\varphi, a)(\xi, i \tau)= & \underbrace{\sum_{k=0}^{N-1} \frac{(-1)^{k}}{k !} \int_{\mathbb{R}} \int_{0}^{\infty} s^{i \eta} \eta^{k} \varphi(s) s M(s, 1)^{k+1}\left(\partial_{\tau}^{k} a\right)(\xi,-M(s, 1) \tau) \frac{d s}{s} d \eta}_{(*)} \\
& +r_{N}(\xi, i \tau),
\end{aligned}
$$

where

$$
\begin{aligned}
& r_{N}(\xi, i \tau):=\int_{0}^{1} \frac{(1-\theta)^{N-1}}{(N-1) !}(-1)^{N} \int_{\mathbb{R}} \int_{0}^{\infty} s^{i \eta} \eta^{N} \varphi(s) s M(s, 1)^{N+1} \\
&\left(\partial_{\tau}^{N} a\right)(\xi,-M(s, 1)(\tau+\theta \eta)) \frac{d s}{s} d \eta d \theta .
\end{aligned}
$$

Now we see that

$$
r_{N}(\xi, i \tau) \in\left\{\begin{array}{l}
S^{\mu-N \ell ; \ell}\left(\mathbb{R}^{n} \times \Gamma_{0} ; E, \tilde{E}\right) \\
S_{V}^{\mu-N \ell ; \ell}\left(\mathbb{R}^{n} \times \mathbb{H}_{0} ; E, \tilde{E}\right)
\end{array}\right.
$$

while $(*)$ equals

$$
\left.\sum_{k=0}^{N-1} \frac{(-i)^{k}}{k !}\left(r \partial_{r}\right)^{k}\left\{\varphi(r) r M(r, 1)^{k+1}\left(\partial_{\tau}^{k} a\right)(\xi,-M(r, 1) \tau)\right\}\right|_{r=1}
$$

which proves the asymptotic expansion.

The proof of b) is analogous, noting that we may rewrite $\tilde{Q}_{\gamma}(\psi, a)$ as

$$
\begin{aligned}
\tilde{Q}_{\gamma}(\psi, a)(\xi, \tau)=\iint_{\mathbb{R}_{+}} e^{-i(s-1) \eta} \frac{\psi(s)}{s} s^{\frac{1}{2}-\gamma} M(s, 1)^{-1} \\
a\left(\xi, \frac{1}{2}-\gamma-M(s, 1)^{-1}(i \tau+i \eta)\right) d s d \eta .
\end{aligned}
$$

Recall that $\psi \in C_{0}^{\infty}\left(\mathbb{R}_{+}\right)$by assumption.

Now let us prove c). Due to the compact support of $\varphi$ and $\psi$ we conclude, that 
the compositions $Q\left(\tilde{Q}_{\gamma}(a)\right)$ and $\tilde{Q}_{\gamma}(Q(a))$ are given as

$$
\begin{aligned}
Q\left(\tilde{Q}_{\gamma}(a)\right)\left(\xi, \frac{1}{2}-\gamma+i \tau\right) & =\int_{\mathbb{R}} \int_{0}^{\infty} s^{i \eta}(\varphi \psi)\left(\frac{1}{s}\right) a\left(\xi, \frac{1}{2}-\gamma+i \tau-i \eta\right) \frac{d s}{s} d \eta \\
\tilde{Q}_{\gamma}(Q(a))(\xi, \tau) & =\int_{\mathbb{R}} \int_{\mathbb{R}} e^{-i s \eta}(\varphi \psi)(-s+1) a(\xi, \tau-\eta) d s d \eta
\end{aligned}
$$

i. e., the first composition equals the Mellin kernel cut-off operator with respect to the weight $\gamma \in \mathbb{R}$ and the function $(\varphi \psi)\left(\frac{1}{r}\right)$ (cf. Definition 5.1), while the second one equals the Fourier kernel cut-off operator with respect to the function $(\varphi \psi)(-r+1)$ (cf. Krainer [23], [24], and Remark 5.2). Now we obtain the desired assertion from Theorem 5.3, noting that $\varphi \psi \equiv 1$ near $r=1$.

5.8 Remark. Let $\varphi, \psi \in C_{0}^{\infty}\left(\mathbb{R}_{+}\right)$such that $\varphi \equiv 1$ and $\psi \equiv 1$ near $r=1$. Then by Theorem 5.7 the mappings $Q: a \mapsto Q(a)$ and $\tilde{Q}_{\gamma}: a \mapsto \tilde{Q}_{\gamma}(a)$ provide isomorphisms

$$
\begin{aligned}
& Q:\left\{\begin{array}{c}
S_{(c l)}^{\mu ; \ell}\left(\mathbb{R}^{n} \times \mathbb{R} ; \mathcal{E}, \tilde{\mathcal{E}}\right) / S^{-\infty}\left(\mathbb{R}^{n} \times \mathbb{R} ; \mathcal{E}, \tilde{\mathcal{E}}\right) \\
\longrightarrow S_{O(c l)}^{\mu ; \ell}\left(\mathbb{R}^{n} \times \mathbb{C} ; \mathcal{E}, \tilde{\mathcal{E}}\right) / S_{O}^{-\infty}\left(\mathbb{R}^{n} \times \mathbb{C} ; \mathcal{E}, \tilde{\mathcal{E}}\right) \\
S_{V(c l)}^{\mu ; \ell}\left(\mathbb{R}^{n} \times \mathbb{H} ; \mathcal{E}, \tilde{\mathcal{E}}\right) / S_{V}^{-\infty}\left(\mathbb{R}^{n} \times \mathbb{H} ; \mathcal{E}, \tilde{\mathcal{E}}\right) \\
\longrightarrow S_{V, O(c l)}^{\mu ; \ell}\left(\mathbb{R}^{n} \times \mathbb{C} ; \mathcal{E}, \tilde{\mathcal{E}}\right) / S_{V, O}^{-\infty}\left(\mathbb{R}^{n} \times \mathbb{C} ; \mathcal{E}, \tilde{\mathcal{E}}\right)
\end{array}\right. \\
& \tilde{Q}_{\gamma}:\left\{\begin{array}{c}
S_{(c l)}^{\mu ; \ell}\left(\mathbb{R}^{n} \times \Gamma_{\frac{1}{2}-\gamma} ; \mathcal{E}, \tilde{\mathcal{E}}\right) / S^{-\infty}\left(\mathbb{R}^{n} \times \Gamma_{\frac{1}{2}-\gamma} ; \mathcal{E}, \tilde{\mathcal{E}}\right) \\
\longrightarrow S_{i O(c l)}^{\mu ; \ell}\left(\mathbb{R}^{n} \times \mathbb{C}^{\prime} \mathcal{E}, \tilde{\mathcal{E}}\right) / S_{i O}^{-\infty}\left(\mathbb{R}^{n} \times \mathbb{C} ; \mathcal{E}, \tilde{\mathcal{E}}\right) \\
S_{V(c l)}^{\mu ; \ell}\left(\mathbb{R}^{n} \times \mathbb{H}_{\frac{1}{2}-\gamma} ; \mathcal{E}, \tilde{\mathcal{E}}\right) / S_{V}^{-\infty}\left(\mathbb{R}^{n} \times \mathbb{H}_{\frac{1}{2}-\gamma} ; \mathcal{E}, \tilde{\mathcal{E}}\right) \\
\longrightarrow S_{V, i O(c l)}^{\mu ; \ell}\left(\mathbb{R}^{n} \times \mathbb{C} ; \mathcal{E}, \tilde{\mathcal{E}}\right) / S_{V, i O}^{-\infty}\left(\mathbb{R}^{n} \times \mathbb{C} ; \mathcal{E}, \tilde{\mathcal{E}}\right) .
\end{array}\right.
\end{aligned}
$$

On the level of quotient spaces, $Q$ and $\tilde{Q}_{\gamma}$ are independent of $\varphi$ and $\psi$, respectively, and we have $\tilde{Q}_{\gamma}=Q^{-1}$ (cf. part c) of Theorem 5.7).

5.9 Theorem. Let $E$ and $\tilde{E}$ be Hilbert spaces with group-actions $\left\{\kappa_{\varrho}\right\}$ and $\left\{\tilde{\kappa}_{\varrho}\right\}$, respectively. Let $\varphi, \psi \in C_{0}^{\infty}\left(\mathbb{R}_{+}\right)$be fixed such that $\varphi \equiv 1$ and $\psi \equiv 1$ near $r=1$.

a) Let $\tilde{a} \in C^{\infty}\left(\mathbb{R}_{+}, S^{\mu ; \ell}\left(\mathbb{R}^{n} \times \mathbb{R} ; E, \tilde{E}\right)\right)$, and define $a(r, \xi, \tau):=\tilde{a}(r, \xi, r \tau)$. Then we have for every $\gamma \in \mathbb{R}$

$$
o p_{r}(a)(\xi)-o p_{M}^{\gamma}(Q(\tilde{a}))(\xi)=o p_{r}\left((1-\varphi)\left(\frac{r^{\prime}}{r}\right) a\right)(\xi)
$$

as operators in $C_{0}^{\infty}\left(\mathbb{R}_{+}, E\right) \longrightarrow C^{\infty}\left(\mathbb{R}_{+}, \tilde{E}\right)$. 
b) Let $\tilde{a} \in C^{\infty}\left(\mathbb{R}_{+}, S^{\mu ; \ell}\left(\mathbb{R}^{n} \times \Gamma_{\frac{1}{2}-\gamma} ; E, \tilde{E}\right)\right)$, and define $a(r, \xi, \tau):=\tilde{Q}_{\gamma}(\tilde{a})(r, \xi, r \tau)$. Then we have

$$
o p_{M}^{\gamma}(\tilde{a})(\xi)-o p_{r}(a)(\xi)=o p_{M}^{\gamma}\left((1-\psi)\left(\frac{r^{\prime}}{r}\right) \tilde{a}\right)(\xi)
$$

as operators in $C_{0}^{\infty}\left(\mathbb{R}_{+}, E\right) \longrightarrow C^{\infty}\left(\mathbb{R}_{+}, \tilde{E}\right)$.

Proof. Let us first prove a). In view of the analyticity of $Q(\tilde{a})$ we conclude from Proposition 4.15 that we only have to consider the case $\gamma=\frac{1}{2}$. We write

$$
a(r, \xi, \tau)=\varphi\left(\frac{r^{\prime}}{r}\right) a(r, \xi, \tau)+(1-\varphi)\left(\frac{r^{\prime}}{r}\right) a(r, \xi, \tau) .
$$

Consequently, for the proof of a) we have to show

$$
\mathrm{op}_{r}\left(\varphi\left(\frac{r^{\prime}}{r}\right) a\right)(\xi)=\mathrm{op}_{M}^{\frac{1}{2}}(Q(\tilde{a}))(\xi)
$$

as operators in $C_{0}^{\infty}\left(\mathbb{R}_{+}, E\right) \longrightarrow C^{\infty}\left(\mathbb{R}_{+}, \tilde{E}\right)$. For $u \in C_{0}^{\infty}\left(\mathbb{R}_{+}, E\right)$ we have with the function $M$ from Lemma 5.5

$$
\begin{gathered}
\left.\operatorname{op}_{r}\left(\varphi\left(\frac{r^{\prime}}{r}\right) a\right)(\xi) u\right)(r)=\iint_{\mathbb{R}_{+}} e^{i\left(r-r^{\prime}\right) \tau} \varphi\left(\frac{r^{\prime}}{r}\right) a(r, \xi, \tau) u\left(r^{\prime}\right) d r^{\prime} d \tau \\
=\int_{\mathbb{R}} \int_{0}^{\infty}\left(\frac{r}{r^{\prime}}\right)^{-i \tau} r^{\prime} M\left(r, r^{\prime}\right) \varphi\left(\frac{r^{\prime}}{r}\right) a\left(r, \xi,-M\left(r, r^{\prime}\right) \tau\right) u\left(r^{\prime}\right) \frac{d r^{\prime}}{r^{\prime}} d \tau .
\end{gathered}
$$

The latter Mellin oscillatory integral equals $\left(\mathrm{op}_{M}^{\frac{1}{2}}(g)(\xi) u\right)(r)$ with the (parameterdependent) Mellin symbol $g\left(r, r^{\prime}, \xi, i \tau\right):=r^{\prime} M\left(r, r^{\prime}\right) \varphi\left(\frac{r^{\prime}}{r}\right) a\left(r, \xi,-M\left(r, r^{\prime}\right) \tau\right)$. For the parameter-dependent Mellin operator $\operatorname{op}_{M}^{\frac{1}{2}}(g)(\xi)$ is properly supported (see, e. g., Schulze [40], [42], [43]), we have $\mathrm{op}_{M}^{\frac{1}{2}}(g)(\xi)=\mathrm{op}_{M}^{\frac{1}{2}}(h)(\xi)$ with the Mellin symbol

$$
\begin{aligned}
h(r, \xi, i \tau) & =r^{i \tau}\left(\operatorname{op}_{M}^{\frac{1}{2}}(g)(\xi) r^{\prime-i \tau}\right)(r) \\
& =\int_{\mathbb{R}} \int_{0}^{\infty} s^{i \eta} g(r, s r, \xi, i(\eta+\tau)) \frac{d s}{s} d \eta .
\end{aligned}
$$

Inserting the definition of $g$ and formula (1) in the proof of Theorem 5.7 yields that $h=Q(\varphi, \tilde{a})=Q(\tilde{a})$ as asserted. This proves a).

The proof of b) is similar. First we decompose $\tilde{a}$ as

$$
\tilde{a}\left(r, \xi, \frac{1}{2}-\gamma+i \tau\right)=\psi\left(\frac{r^{\prime}}{r}\right) \tilde{a}\left(r, \xi, \frac{1}{2}-\gamma+i \tau\right)+\left(1-\psi\left(\frac{r^{\prime}}{r}\right)\right) \tilde{a}\left(r, \xi, \frac{1}{2}-\gamma+i \tau\right)
$$


which reduces the proof to show that

$$
\operatorname{op}_{M}^{\gamma}\left(\psi\left(\frac{r^{\prime}}{r}\right) \tilde{a}\right)(\xi)=\operatorname{op}_{r}(a)(\xi)
$$

as operators in $C_{0}^{\infty}\left(\mathbb{R}_{+}, E\right) \longrightarrow C^{\infty}\left(\mathbb{R}_{+}, \tilde{E}\right)$. With the function $M$ from Lemma 5.5 we may write for $u \in C_{0}^{\infty}\left(\mathbb{R}_{+}, E\right)$

$$
\begin{aligned}
& \left(\operatorname{op}_{M}^{\gamma}\left(\psi\left(\frac{r^{\prime}}{r}\right) \tilde{a}\right)(\xi) u\right)(r)=\int_{\mathbb{R}} \int_{0}^{\infty}\left(\frac{r}{r^{\prime}}\right)^{-i \tau}\left(\frac{r^{\prime}}{r}\right)^{\frac{1}{2}-\gamma} \psi\left(\frac{r^{\prime}}{r}\right) \tilde{a}(r, \xi, i \tau) u\left(r^{\prime}\right) \frac{d r^{\prime}}{r^{\prime}} d \tau \\
& =\int_{\mathbb{R}_{\mathbb{R}_{+}}} e^{i\left(r-r^{\prime}\right) \tau} \frac{1}{r^{\prime} M\left(r, r^{\prime}\right)}\left(\frac{r^{\prime}}{r}\right)^{\frac{1}{2}-\gamma} \psi\left(\frac{r^{\prime}}{r}\right) \tilde{a}\left(r, \xi,-M\left(r, r^{\prime}\right)^{-1} i \tau\right) u\left(r^{\prime}\right) d r^{\prime} d \tau,
\end{aligned}
$$

i. e., we have a representation of the Mellin operator by means of a pseudodifferential operator based on the Fourier transform with the parameter-dependent symbol $g\left(r, r^{\prime}, \xi, \tau\right):=\frac{1}{r^{\prime} M\left(r, r^{\prime}\right)}\left(\frac{r^{\prime}}{r}\right)^{\frac{1}{2}-\gamma} \psi\left(\frac{r^{\prime}}{r}\right) \tilde{a}\left(r, \xi,-M\left(r, r^{\prime}\right)^{-1} i \tau\right)$. For $\operatorname{op}_{r}(g)(\xi)$ is properly supported we obtain that $\mathrm{op}_{r}(g)(\xi)=\mathrm{op}_{r}(h)(\xi)$ with $h$ given as

$$
\begin{aligned}
h(r, \xi, \tau) & =e^{-i r \tau}\left(\operatorname{op}_{r}(g)(\xi) e^{i r^{\prime} \tau}\right)(r) \\
& =\int_{\mathbb{R}_{\mathbb{R}_{+}}} e^{-i\left(r^{\prime}-1\right) \eta} g\left(r, r r^{\prime}, \xi, \frac{\eta}{r}+\tau\right) d r^{\prime} d \eta,
\end{aligned}
$$

i. e., from the definition of $g$ and formula (2) in the proof of Theorem 5.7 we see that $h=a$ as asserted.

5.10 Remark. Theorem 5.9 motivates the name "Mellin quantization" for the operator $Q$ and "inverse Mellin quantization" for $\tilde{Q}$. Together with Theorem 5.7 it follows, that modulo "smoothing" (Volterra) operators we obtain isomorphisms between Fourier pseudodifferential operators with degenerate (Volterra) symbols on the half-axis and (Volterra) Mellin pseudodifferential operators.

Notice, in particular, that if in a) or b) the dependence of the symbol $\tilde{a}$ on $r \in \mathbb{R}_{+}$ is $C_{B}^{\infty}\left(\overline{\mathbb{R}}_{+}, \cdot\right)$, then so is also the dependence of $Q(\tilde{a})$ or $\tilde{Q}(\tilde{a})$, respectively.

The technique to express Mellin quantization via explicit Mellin oscillatory integral formulas was used first in concrete situations, especially edge symbolic calculus, by Gil, Seiler, and Schulze [14], [15], see also Seiler [46].

\section{Parabolicity and Volterra parametrices}

\subsection{Ellipticity and parabolicity on symbolic level}

6.1 Remark. In the present section we recall the notion of ellipticity for general and holomorphic operator-valued Mellin symbols, and we introduce the notion of 
parabolicity for Volterra symbols. In particular, we focus on the study of symbolic invertibility within the corresponding classes up to lower order terms, which is the starting point for the construction of parametrices in the next section.

Notice, in particular, that the study of parabolicity requires a more subtle treatment on the side of the covariables than the study of ellipticity for there is the additional analyticity condition involved. Techniques involving excision functions have to be replaced; the translation operator in Volterra symbols provides the appropriate tool to overcome these difficulties.

6.2 Definition. Let $E$ and $\tilde{E}$ be Hilbert spaces with group-actions $\left\{\kappa_{\varrho}\right\}$ and $\left\{\tilde{\kappa}_{\varrho}\right\}$, respectively.

a) A symbol $a\left(r, \xi, \frac{1}{2}-\gamma+i \tau\right) \in M_{\gamma} S^{\mu ; \ell}\left(\mathbb{R}_{+} \times \mathbb{R}^{n} \times \Gamma_{\frac{1}{2}-\gamma} ; E, \tilde{E}\right)$ is called elliptic if there exists $R>0$ such that for $(\xi, \tau) \in \mathbb{R}^{n} \times \mathbb{R}^{2}$ with $|(\xi, \tau)|_{\ell} \geq R$ and all $r \in \mathbb{R}_{+}$there exists $a\left(r, \xi, \frac{1}{2}-\gamma+i \tau\right)^{-1} \in \mathcal{L}(\tilde{E}, E)$, and

$$
\sup \left\{\left\|\kappa_{\langle\xi, \tau\rangle_{\ell}}^{-1}\left(a\left(r, \xi, \frac{1}{2}-\gamma+i \tau\right)^{-1}\right) \tilde{\kappa}_{\langle\xi, \tau\rangle_{\ell}}\right\|\langle\xi, \tau\rangle_{\ell}^{\mu} ; r \in \mathbb{R}_{+},|(\xi, \tau)|_{\ell} \geq R\right\}<\infty .
$$

b) A symbol $a\left(r, \xi, \frac{1}{2}-\gamma+\zeta\right) \in M_{\gamma} S_{V}^{\mu, \ell}\left(\mathbb{R}_{+} \times \mathbb{R}^{n} \times \mathbb{H}_{\frac{1}{2}-\gamma} ; E, \tilde{E}\right)$ is called parabolic if there exists $R>0$ such that for $(\xi, \zeta) \in \mathbb{R}^{n} \times \mathbb{H}_{0}^{2}$ with $|(\xi, \zeta)|_{\ell} \geq R$ and all $r \in \mathbb{R}_{+}$there exists $a\left(r, \xi, \frac{1}{2}-\gamma+\zeta\right)^{-1} \in \mathcal{L}(\tilde{E}, E)$, and

$$
\sup \left\{\left\|\kappa_{\langle\xi, \zeta\rangle_{\ell}}^{-1}\left(a\left(r, \xi, \frac{1}{2}-\gamma+\zeta\right)^{-1}\right) \tilde{\kappa}_{\langle\xi, \zeta\rangle_{\ell}}\right\|\langle\xi, \zeta\rangle_{\ell}^{\mu} ; r \in \mathbb{R}_{+},|(\xi, \zeta)|_{\ell} \geq R\right\}<\infty .
$$

6.3 Proposition. Let $a(r, \xi, z) \in M_{\gamma} S^{\mu ; \ell}\left(\mathbb{R}_{+} \times \mathbb{R}^{n} \times \Gamma_{\frac{1}{2}-\gamma} ; E, \tilde{E}\right)$. The following are equivalent:

a) $a(r, \xi, z)$ is elliptic.

b) There exists $b \in M_{\gamma} S^{-\mu ; \ell}\left(\mathbb{R}_{+} \times \mathbb{R}^{n} \times \Gamma_{\frac{1}{2}-\gamma} ; \tilde{E}, E\right)$ such that $a b-1 \in$ $M_{\gamma} S^{-\varepsilon ; \ell}\left(\mathbb{R}_{+} \times \mathbb{R}^{n} \times \Gamma_{\frac{1}{2}-\gamma} ; \tilde{E}, \tilde{E}\right)$ and $b a-1 \in M_{\gamma} S^{-\varepsilon ; \ell}\left(\mathbb{R}_{+} \times \mathbb{R}^{n} \times \Gamma_{\frac{1}{2}-\gamma} ; E, E\right)$ for some $\varepsilon>0$. (It is possible to choose $\varepsilon=1$.)

In case of a classical symbol $a(r, \xi, z), a)$ and $b)$ are equivalent to the following:

c) The principal symbol $a_{(\mu)}\left(r, \xi, \frac{1}{2}-\gamma+i \tau\right)$ is invertible for $(\xi, \tau) \in\left(\mathbb{R}^{n} \times \mathbb{R}\right) \backslash 0$ and all $r \in \mathbb{R}_{+}$, and we have

$$
\sup \left\{\left\|a_{(\mu)}\left(r, \xi, \frac{1}{2}-\gamma+i \tau\right)^{-1}\right\|_{\mathcal{L}(\tilde{E}, E)} ; r \in \mathbb{R}_{+},|\xi, \tau|_{\ell}=1\right\}<\infty .
$$


d) There exists $b \in M_{\gamma} S_{c l}^{-\mu ; \ell}\left(\mathbb{R}_{+} \times \mathbb{R}^{n} \times \Gamma_{\frac{1}{2}-\gamma} ; \tilde{E}, E\right)$ such that $a b-1 \in$ $M_{\gamma} S_{c l}^{-1 ; \ell}\left(\mathbb{R}_{+} \times \mathbb{R}^{n} \times \Gamma_{\frac{1}{2}-\gamma} ; \tilde{E}, \tilde{E}\right)$ and $b a-1 \in M_{\gamma} S_{c l}^{-1 ; \ell}\left(\mathbb{R}_{+} \times \mathbb{R}^{n} \times \Gamma_{\frac{1}{2}-\gamma} ; E, E\right)$.

Moreover, if $a(r, \xi, z) \in M_{\gamma} S_{V}^{\mu ; \ell}\left(\mathbb{R}_{+} \times \mathbb{R}^{n} \times \mathbb{H}_{\frac{1}{2}-\gamma} ; E, \tilde{E}\right)$, the following are equivalent:

i) $a(r, \xi, z)$ is parabolic.

ii) There exists $b \in M_{\gamma} S_{V}^{-\mu ; \ell}\left(\mathbb{R}_{+} \times \mathbb{R}^{n} \times \mathbb{H}_{\frac{1}{2}-\gamma} ; \tilde{E}, E\right)$ such that $a b-1 \in$ $M_{\gamma} S_{V}^{-\varepsilon ; \ell}\left(\mathbb{R}_{+} \times \mathbb{R}^{n} \times \mathbb{H}_{\frac{1}{2}-\gamma} ; \tilde{E}, \tilde{E}\right)$ and $b a-1 \in M_{\gamma} S_{V}^{-\varepsilon ; \ell}\left(\mathbb{R}_{+} \times \mathbb{R}^{n} \times \mathbb{H}_{\frac{1}{2}-\gamma} ; E, E\right)$ for some $\varepsilon>0$. (It is possible to choose $\varepsilon=1$.)

In case of a classical Volterra symbol $a(r, \xi, z)$, i) and ii) are equivalent to the following:

iii) The principal symbol $a_{(\mu)}\left(r, \xi, \frac{1}{2}-\gamma+\zeta\right)$ is invertible for $(\xi, \zeta) \in\left(\mathbb{R}^{n} \times \mathbb{H}_{0}\right) \backslash 0$ and all $r \in \mathbb{R}_{+}$, and we have

$$
\sup \left\{\left\|a_{(\mu)}\left(r, \xi, \frac{1}{2}-\gamma+\zeta\right)^{-1}\right\|_{\mathcal{L}(\tilde{E}, E)} ; r \in \mathbb{R}_{+},|\xi, \zeta|_{\ell}=1\right\}<\infty .
$$

iv) There exists $b \in M_{\gamma} S_{V c l}^{-\mu ; \ell}\left(\mathbb{R}_{+} \times \mathbb{R}^{n} \times \mathbb{H}_{\frac{1}{2}-\gamma} ; \tilde{E}, E\right)$ such that $a b-1 \in$ $M_{\gamma} S_{V c l}^{-1 ; \ell}\left(\mathbb{R}_{+} \times \mathbb{R}^{n} \times \mathbb{H}_{\frac{1}{2}-\gamma} ; \tilde{E}, \tilde{E}\right)$ and $b a-1 \in M_{\gamma} S_{V c l}^{-1 ; \ell}\left(\mathbb{R}_{+} \times \mathbb{R}^{n} \times \mathbb{H}_{\frac{1}{2}-\gamma} ; E, E\right)$.

The ellipticity and parabolicity conditions are invariant with respect to additive perturbations of (Volterra) Mellin symbols of lower order.

Proof. Note that in view of Definition 6.2 the conditions in b) and ii) are clearly sufficient for the ellipticity or parabolicity of the symbol $a$, respectively. Moreover, the implications d) to c), b), as well as iv) to iii), ii) are evident.

The implications a) to c) and i) to iii) in the classical case are due to the fact that the invertibility conditions (6.i), (6.ii) in Definition 6.2 are easily seen to be invariant with respect to additive perturbations of lower order. To make the argument more precise, this invariance reduces to require the invertibility for $\chi a_{(\mu)}$ instead of the full symbol $a$, where $\chi$ is a smooth 0-excision function in the covariables $(\xi, \tau) \in \mathbb{R}^{n} \times \mathbb{R}$ or $(\xi, \zeta) \in \mathbb{R}^{n} \times \mathbb{H}_{0}$, respectively. In view of the homogeneity of the principal component $a_{(\mu)}$ the conditions $(6 . \mathrm{i})$, (6.ii) then reduce to (6.iii), (6.iv), respectively.

What remains to show are the implications a) to b), c) to d), as well as i) to ii), and iii) to iv). 
Let us assume that a) or c) holds for the Mellin symbol $a$, and let $\chi \in C^{\infty}\left(\mathbb{R}^{n} \times \mathbb{R}\right)$ be a 0 -excision function with $\chi \equiv 0$ for $|\xi, \tau|_{\ell} \leq R+1$ and $\chi \equiv 1$ for $|\xi, \tau|_{\ell} \geq$ $R+2$, where $R>0$ is chosen from the ellipticity condition in Definition 6.2. For $\left(r, \xi, \frac{1}{2}-\gamma+i \tau\right) \in \mathbb{R}_{+} \times \mathbb{R}^{n} \times \Gamma_{\frac{1}{2}-\gamma}$ define

$$
b\left(r, \xi, \frac{1}{2}-\gamma+i \tau\right):= \begin{cases}\chi(\xi, \tau)\left(a\left(r, \xi, \frac{1}{2}-\gamma+i \tau\right)\right)^{-1} & \text { in the general case } \\ \chi(\xi, \tau)\left(a_{(\mu)}\left(r, \xi, \frac{1}{2}-\gamma+i \tau\right)\right)^{-1} & \text { in the classical case. }\end{cases}
$$

Thus we see that $b \in M_{\gamma} S_{(c l)}^{-\mu ; \ell}\left(\mathbb{R}_{+} \times \mathbb{R}^{n} \times \Gamma_{\frac{1}{2}-\gamma} ; \tilde{E}, E\right)$, and moreover $a b-1 \in$ $M_{\gamma} S_{(c l)}^{-1 ; \ell}\left(\mathbb{R}_{+} \times \mathbb{R}^{n} \times \Gamma_{\frac{1}{2}-\gamma} ; \tilde{E}, \tilde{E}\right)$ and $b a-1 \in M_{\gamma} S_{(c l)}^{-1 ; \ell}\left(\mathbb{R}_{+} \times \mathbb{R}^{n} \times \Gamma_{\frac{1}{2}-\gamma} ; E, E\right)$ as desired.

Now assume that i) or iii) holds for the Volterra Mellin symbol $a$, and let $R>0$ be chosen according to the parabolicity condition in Definition 6.2. For $\left(r, \xi, \frac{1}{2}-\right.$ $\gamma+\zeta) \in \mathbb{R}_{+} \times \mathbb{R}^{n} \times \mathbb{H}_{\frac{1}{2}-\gamma}$, where $\zeta \in \mathbb{H}_{0}$, define

$$
b\left(r, \xi, \frac{1}{2}-\gamma+\zeta\right):= \begin{cases}\left(a\left(r, \xi, \frac{1}{2}-\gamma+R+\zeta\right)\right)^{-1} & \text { in the general case } \\ \left(a_{(\mu)}\left(r, \xi, \frac{1}{2}-\gamma+1+\zeta\right)\right)^{-1} & \text { in the classical case. }\end{cases}
$$

From the investigations on the translation operator in Proposition 3.20 and Theorem 3.22 we conclude that $b \in M_{\gamma} S_{V(c l)}^{-\mu ; \ell}\left(\mathbb{R}_{+} \times \mathbb{R}^{n} \times \mathbb{H}_{\frac{1}{2}-\gamma} ; \tilde{E}, E\right)$, and moreover $a b-1 \in M_{\gamma} S_{V(c l)}^{-1 ; \ell}\left(\mathbb{R}_{+} \times \mathbb{R}^{n} \times \mathbb{H}_{\frac{1}{2}-\gamma} ; \tilde{E}, \tilde{E}\right)$ and $b a-1 \in M_{\gamma} S_{V(c l)}^{-1 ; \ell}\left(\mathbb{R}_{+} \times \mathbb{R}^{n} \times\right.$ $\left.\mathbb{H}_{\frac{1}{2}-\gamma} ; E, E\right)$.

6.4 Definition. Let $E$ and $\tilde{E}$ be Hilbert spaces with group-actions $\left\{\kappa_{\varrho}\right\}$ and $\left\{\tilde{\kappa}_{\varrho}\right\}$, respectively.

a) A symbol $a(r, \xi, z) \in M S_{O}^{\mu ; \ell}\left(\overline{\mathbb{R}}_{+} \times \mathbb{R}^{n} \times \mathbb{C} ; E, \tilde{E}\right)$ is called elliptic if there exists $\gamma \in \mathbb{R}$ as well as $R>0$ such that for $(\xi, \tau) \in \mathbb{R}^{n} \times \mathbb{R}$ with $|(\xi, \tau)|_{\ell} \geq R$ and all $r \in \overline{\mathbb{R}}_{+}$there exists $a\left(r, \xi, \frac{1}{2}-\gamma+i \tau\right)^{-1} \in \mathcal{L}(\tilde{E}, E)$, and

$$
\sup \left\{\left\|\kappa_{\langle\xi, \tau\rangle_{\ell}}^{-1}\left(a\left(r, \xi, \frac{1}{2}-\gamma+i \tau\right)^{-1}\right) \tilde{\kappa}_{\langle\xi, \tau\rangle_{\ell}}\right\|\langle\xi, \tau\rangle_{\ell}^{\mu} ; r \in \overline{\mathbb{R}}_{+},|(\xi, \tau)|_{\ell} \geq R\right\}<\infty .
$$

b) A symbol $a(r, \xi, z) \in M S_{V O O}^{\mu ; \ell}\left(\overline{\mathbb{R}}_{+} \times \mathbb{R}^{n} \times \mathbb{C} ; E, \tilde{E}\right)$ is called parabolic if there exists $\gamma \in \mathbb{R}$ as well as $R>0$ such that for $(\xi, \zeta) \in \mathbb{R}^{n} \times \mathbb{H}_{0}$ with $|(\xi, \zeta)|_{\ell} \geq R$ and all $r \in \overline{\mathbb{R}}_{+}$there exists $a\left(r, \xi, \frac{1}{2}-\gamma+\zeta\right)^{-1} \in \mathcal{L}(\tilde{E}, E)$, and

$$
\sup \left\{\left\|\kappa_{\langle\xi, \zeta\rangle_{\ell}}^{-1}\left(a\left(r, \xi, \frac{1}{2}-\gamma+\zeta\right)^{-1}\right) \tilde{\kappa}_{\langle\xi, \zeta\rangle_{\ell}}\right\|\langle\xi, \zeta\rangle_{\ell}^{\mu} ; r \in \overline{\mathbb{R}}_{+},|(\xi, \zeta)|_{\ell} \geq R\right\}<\infty .
$$


6.5 Remark. The ellipticity and parabolicity condition for a holomorphic (Volterra) Mellin symbol $a(r, \xi, z)$ means that its restriction in the covariable $z \in \mathbb{C}$ to a weight line or half plane is required to be elliptic or parabolic in the sense of Definition 6.2, where the invertibility is additionally assumed to hold up to the origin $r=0$.

As in Proposition 6.3, it is easy to see that in case of a classical symbol $a(r, \xi, z)$ the ellipticity condition $(6 . \mathrm{v})$ is equivalent to require the invertibility of the principal symbol $a_{(\mu)}\left(r, \xi, \frac{1}{2}-\gamma+i \tau\right)$ for $0 \neq(\xi, \tau) \in \mathbb{R}^{n} \times \mathbb{R}$ and all $r \in \overline{\mathbb{R}}_{+}$with

$$
\sup \left\{\left\|a_{(\mu)}\left(r, \xi, \frac{1}{2}-\gamma+i \tau\right)^{-1}\right\|_{\mathcal{L}(\tilde{E}, E)} ; r \in \overline{\mathbb{R}}_{+},|\xi, \tau|_{\ell}=1\right\}<\infty .
$$

Analogously, the parabolicity condition (6.vi) for a classical holomorphic Volterra Mellin symbol is equivalent to require the invertibility of the principal symbol $a_{(\mu)}\left(r, \xi, \frac{1}{2}-\gamma+\zeta\right)$ for $0 \neq(\xi, \zeta) \in \mathbb{R}^{n} \times \mathbb{H}_{0}$ and all $r \in \overline{\mathbb{R}}_{+}$with

$$
\sup \left\{\left\|a_{(\mu)}\left(r, \xi, \frac{1}{2}-\gamma+\zeta\right)^{-1}\right\|_{\mathcal{L}(\tilde{E}, E)} ; r \in \overline{\mathbb{R}}_{+},|\xi, \zeta|_{\ell}=1\right\}<\infty
$$

From the investigations on holomorphic (Volterra) symbols in Section 3.4, especially Proposition 3.25, we conclude that the ellipticity and parabolicity condition in Definition 6.4 are in fact independent of the choice of the particular weight line of half-plane, i. e., once they are fulfilled for some $\gamma \in \mathbb{R}$ they hold for all $\gamma \in \mathbb{R}$. This is due to the fact that all restrictions to weight lines or half-planes coincide up to lower order terms.

The following theorem establishes the equivalence of ellipticity and parabolicity for holomorphic (Volterra) Mellin symbols to the corresponding invertibility result within the appropriate symbol classes up to lower order terms.

6.6 Theorem. Let $a(r, \xi, z) \in M S_{O(c l)}^{\mu ; \ell}\left(\overline{\mathbb{R}}_{+} \times \mathbb{R}^{n} \times \mathbb{C} ; E, \tilde{E}\right)$. The following are equivalent:

a) $a(r, \xi, z)$ is elliptic.

b) There exists $b \in M S_{O(c l)}^{-\mu ; \ell}\left(\overline{\mathbb{R}}_{+} \times \mathbb{R}^{n} \times \mathbb{C} ; \tilde{E}, E\right)$ such that $a b-1 \in M S_{O(c l)}^{-1 ; \ell}\left(\overline{\mathbb{R}}_{+} \times\right.$ $\left.\mathbb{R}^{n} \times \mathbb{C} ; \tilde{E}, \tilde{E}\right)$ and $b a-1 \in M S_{O(c l)}^{-1 ; \ell}\left(\overline{\mathbb{R}}_{+} \times \mathbb{R}^{n} \times \mathbb{C} ; E, E\right)$.

Moreover, for $a(r, \xi, z) \in M S_{V, O(c l)}^{\mu ; \ell}\left(\overline{\mathbb{R}}_{+} \times \mathbb{R}^{n} \times \mathbb{C} ; E, \tilde{E}\right)$ the following are equivalent:

i) $a(r, \xi, z)$ is parabolic.

ii) There exists $b \in M S_{V, O(c l)}^{-\mu ; \ell}\left(\overline{\mathbb{R}}_{+} \times \mathbb{R}^{n} \times \mathbb{C} ; \tilde{E}, E\right)$ such that $a b-1 \in$ $M S_{V, O(c l)}^{-1 ; \ell}\left(\overline{\mathbb{R}}_{+} \times \mathbb{R}^{n} \times \mathbb{C} ; \tilde{E}, \tilde{E}\right)$ and $b a-1 \in M S_{V, O(c l)}^{-1 ; \ell}\left(\overline{\mathbb{R}}_{+} \times \mathbb{R}^{n} \times \mathbb{C} ; E, E\right)$. 
Proof. Clearly, the existence of the symbol $b(r, \xi, z)$ is sufficient for the ellipticity or parabolicity of $a(r, \xi, z)$, respectively.

Now assume that $a(r, \xi, z)$ is elliptic or parabolic, respectively. Following the lines of the proof of Proposition 6.3 it is easy to see that for some fixed $\gamma \in \mathbb{R}$ there exists

$$
\tilde{b}(r, \xi, z) \in \begin{cases}C_{B}^{\infty}\left(\overline{\mathbb{R}}_{+}, S_{(c l)}^{-\mu, \ell}\left(\mathbb{R}^{n} \times \Gamma_{\frac{1}{2}-\gamma} ; \tilde{E}, E\right)\right) & \text { under assumption a) } \\ C_{B}^{\infty}\left(\overline{\mathbb{R}}_{+}, S_{V(c l)}^{-\mu ; \ell}\left(\mathbb{R}^{n} \times \mathbb{H}_{\frac{1}{2}-\gamma} ; \tilde{E}, E\right)\right) & \text { under assumption i })\end{cases}
$$

such that

$$
\left.\begin{array}{r}
\left(\left.a\right|_{\Gamma_{\frac{1}{2}-\gamma}}\right) \tilde{b}-1 \in C_{B}^{\infty}\left(\overline{\mathbb{R}}_{+}, S_{(c l)}^{-1 ; \ell}\left(\mathbb{R}^{n} \times \Gamma_{\frac{1}{2}-\gamma} ; \tilde{E}, \tilde{E}\right)\right) \\
\tilde{b}\left(\left.a\right|_{\Gamma_{\frac{1}{2}-\gamma}}\right)-1 \in C_{B}^{\infty}\left(\overline{\mathbb{R}}_{+}, S_{(c l)}^{-1 ; \ell}\left(\mathbb{R}^{n} \times \Gamma_{\frac{1}{2}-\gamma} ; E, E\right)\right)
\end{array}\right\} \quad \text { under assumption a), }
$$

According to the properties of the Mellin kernel cut-off operator $H_{\gamma}$ given in Theorem 5.3, we may replace $\tilde{b}$ in the above identities by $b:=H_{\gamma}(\varphi) \tilde{b}$, where $\varphi \in C_{0}^{\infty}\left(\mathbb{R}_{+}\right)$with $\varphi \equiv 1$ near $r=1$; notice, in particular, that $\tilde{b}-b$ is of order $-\infty$ with respect to the weight line $\Gamma_{\frac{1}{2}-\gamma}$ or half-plane $\mathbb{H}_{\frac{1}{2}-\gamma}$, respectively, and we have $b \in M S_{(V,) O(c l)}^{-\mu, \ell}\left(\overline{\mathbb{R}}_{+} \times \mathbb{R}^{n} \times \mathbb{C} ; \tilde{E}, E\right)$. Using Proposition 3.25 we now conclude that $b$ fulfills the assertion in b) or ii), respectively.

\subsection{The parametrix construction}

6.7 Remark. Using the algebraic properties of the (Volterra) Mellin calculus, i. e., the analysis of compositions from Theorem 4.5 and Theorem 4.20, as well as the analysis of ellipticity and parabolicity on symbolic level from the preceding section, we are now in the position to establish the existence of (Volterra) parametrices within the calculus for elliptic and parabolic Mellin operators. The possibility to carry out asymptotic expansions within all symbol classes under consideration, see, e. g., Remark 3.8 (for general symbols) and Remark 5.4 (for Volterra and holomorphic (Volterra) symbols), enables us to employ the classical formal Neumann series argument in the construction of the parametrices.

6.8 Theorem. Let $E$ and $\tilde{E}$ be Hilbert spaces with group-actions $\left\{\kappa_{\varrho}\right\}$ and $\left\{\tilde{\kappa}_{\varrho}\right\}$, respectively, and let

$$
a(r, \xi, z) \in\left\{\begin{array}{l}
M_{\gamma} S_{(c l)}^{\mu ; \ell}\left(\mathbb{R}_{+} \times \mathbb{R}^{n} \times \Gamma_{\frac{1}{2}-\gamma} ; E, \tilde{E}\right) \\
M S_{O(c l)}^{\mu ; \ell}\left(\overline{\mathbb{R}}_{+} \times \mathbb{R}^{n} \times \mathbb{C} ; E, \tilde{E}\right) .
\end{array}\right.
$$

The following are equivalent: 
a) $a(r, \xi, z)$ is elliptic.

b) There exists

$$
b(r, \xi, z) \in\left\{\begin{array}{l}
M_{\gamma} S_{(c l)}^{-\mu ; \ell}\left(\mathbb{R}_{+} \times \mathbb{R}^{n} \times \Gamma_{\frac{1}{2}-\gamma} ; \tilde{E}, E\right) \\
M S_{O(c l)}^{-\mu ; \ell}\left(\overline{\mathbb{R}}_{+} \times \mathbb{R}^{n} \times \mathbb{C} ; \tilde{E}, E\right)
\end{array}\right.
$$

such that

$$
\begin{aligned}
& a \# b-1 \in\left\{\begin{array}{l}
M_{\gamma} S^{-\infty}\left(\mathbb{R}_{+} \times \mathbb{R}^{n} \times \Gamma_{\frac{1}{2}-\gamma} ; \tilde{E}, \tilde{E}\right) \\
M S_{O}^{-\infty}\left(\overline{\mathbb{R}}_{+} \times \mathbb{R}^{n} \times \mathbb{C} ; \tilde{E}, \tilde{E}\right),
\end{array}\right. \\
& b \# a-1 \in\left\{\begin{array}{l}
M_{\gamma} S^{-\infty}\left(\mathbb{R}_{+} \times \mathbb{R}^{n} \times \Gamma_{\frac{1}{2}-\gamma} ; E, E\right) \\
M S_{O}^{-\infty}\left(\overline{\mathbb{R}}_{+} \times \mathbb{R}^{n} \times \mathbb{C} ; E, E\right) .
\end{array}\right.
\end{aligned}
$$

In particular, we have

$$
\begin{aligned}
& o p_{M}^{\gamma}(a)(\xi) o p_{M}^{\gamma}(b)(\xi)-1 \in\left\{\begin{array}{l}
M_{\gamma} L^{-\infty}\left(\mathbb{R}_{+} ; \mathbb{R}^{n} ; \tilde{E}, \tilde{E}\right) \\
M_{O} L^{-\infty}\left(\mathbb{R}_{+} ; \mathbb{R}^{n} ; \tilde{E}, \tilde{E}\right),
\end{array}\right. \\
& \text { op }_{M}^{\gamma}(b)(\xi) o p_{M}^{\gamma}(a)(\xi)-1 \in\left\{\begin{array}{l}
M_{\gamma} L^{-\infty}\left(\mathbb{R}_{+} ; \mathbb{R}^{n} ; E, E\right) \\
M_{O} L^{-\infty}\left(\mathbb{R}_{+} ; \mathbb{R}^{n} ; E, E\right) .
\end{array}\right.
\end{aligned}
$$

Moreover, let

$$
a(r, \xi, z) \in\left\{\begin{array}{l}
M_{\gamma} S_{V(c l)}^{\mu ; \ell}\left(\mathbb{R}_{+} \times \mathbb{R}^{n} \times \mathbb{H}_{\frac{1}{2}-\gamma} ; E, \tilde{E}\right) \\
M S_{V, O(c l)}^{\mu ; \ell}\left(\overline{\mathbb{R}}_{+} \times \mathbb{R}^{n} \times \mathbb{C} ; E, \tilde{E}\right) .
\end{array}\right.
$$

Then the following are equivalent:

i) $a(r, \xi, z)$ is parabolic.

ii) There exists

$$
b(r, \xi, z) \in\left\{\begin{array}{l}
M_{\gamma} S_{V(c l)}^{-\mu ; \ell}\left(\mathbb{R}_{+} \times \mathbb{R}^{n} \times \mathbb{H}_{\frac{1}{2}-\gamma} ; \tilde{E}, E\right) \\
M S_{V, O(c l)}^{-\mu ; \ell}\left(\overline{\mathbb{R}}_{+} \times \mathbb{R}^{n} \times \mathbb{C} ; \tilde{E}, E\right)
\end{array}\right.
$$

such that

$$
\begin{aligned}
& a \# b-1 \in\left\{\begin{array}{l}
M_{\gamma} S_{V}^{-\infty}\left(\mathbb{R}_{+} \times \mathbb{R}^{n} \times \mathbb{H}_{\frac{1}{2}-\gamma} ; \tilde{E}, \tilde{E}\right) \\
M_{V, O}^{-\infty}\left(\overline{\mathbb{R}}_{+} \times \mathbb{R}^{n} \times \mathbb{C} ; \tilde{E}, \tilde{E}\right),
\end{array}\right. \\
& b \# a-1 \in\left\{\begin{array}{l}
M_{\gamma} S_{V}^{-\infty}\left(\mathbb{R}_{+} \times \mathbb{R}^{n} \times \mathbb{H}_{\frac{1}{2}-\gamma} ; E, E\right) \\
M S_{V, O}^{-\infty}\left(\overline{\mathbb{R}}_{+} \times \mathbb{R}^{n} \times \mathbb{C} ; E, E\right) .
\end{array}\right.
\end{aligned}
$$


In particular, we have

$$
\begin{aligned}
& \operatorname{op}_{M}^{\gamma}(a)(\xi) \operatorname{op}_{M}^{\gamma}(b)(\xi)-1 \in\left\{\begin{array}{l}
M_{\gamma} L_{V}^{-\infty}\left(\mathbb{R}_{+} ; \mathbb{R}^{n} ; \tilde{E}, \tilde{E}\right) \\
M_{V, O} L^{-\infty}\left(\mathbb{R}_{+} ; \mathbb{R}^{n} ; \tilde{E}, \tilde{E}\right),
\end{array}\right. \\
& \operatorname{op}_{M}^{\gamma}(b)(\xi) \operatorname{op}_{M}^{\gamma}(a)(\xi)-1 \in\left\{\begin{array}{l}
M_{\gamma} L_{V}^{-\infty}\left(\mathbb{R}_{+} ; \mathbb{R}^{n} ; E, E\right) \\
M_{V, O} L^{-\infty}\left(\mathbb{R}_{+} ; \mathbb{R}^{n} ; E, E\right) .
\end{array}\right.
\end{aligned}
$$

Proof. Assume first that we are given a symbol $b(r, \xi, z)$ satisfying b) or ii), respectively. In view of the asymptotic expansion of the Leibniz-product from Theorem 4.5 and Theorem 4.20 we see that $a \# b=a b$ and $b \# a=b a$ up to terms of order -1 , and, consequently, $a b-1$ and $b a-1$ are of order -1 , which implies the ellipticity or parabolicity of the symbol $a(r, \xi, z)$, respectively, according to Proposition 6.3 and Theorem 6.6.

Now assume that $a(r, \xi, z)$ is elliptic or parabolic. From Proposition 6.3 and Theorem 6.6 we conclude that there exists a symbol

$$
\tilde{b}(r, \xi, z) \in\left\{\begin{array}{c}
M_{\gamma} S_{(c l)}^{-\mu ; \ell}\left(\mathbb{R}_{+} \times \mathbb{R}^{n} \times \Gamma_{\frac{1}{2}-\gamma} ; \tilde{E}, E\right) \\
M S_{O(c l)}^{-\mu ; \ell}\left(\overline{\mathbb{R}}_{+} \times \mathbb{R}^{n} \times \mathbb{C} ; \tilde{E}, E\right) \\
M_{\gamma} S_{V(c l)}^{-\mu ; \ell}\left(\mathbb{R}_{+} \times \mathbb{R}^{n} \times \mathbb{H}_{\frac{1}{2}-\gamma} ; \tilde{E}, E\right) \\
M S_{V, O(c l)}^{-\mu ; \ell}\left(\mathbb{R}_{+} \times \mathbb{R}^{n} \times \mathbb{C} ; \tilde{E}, E\right)
\end{array}\right\} \quad \text { in the cases a), }
$$

such that $a \tilde{b}-1$ and $\tilde{b} a-1$ are of order -1 in the corresponding symbol classes, and thus the same holds true for $a \# \tilde{b}-1$ and $\tilde{b} \# a-1$ by the asymptotic expansion of the Leibniz-product.

Now assume, for instance, that $a \# \tilde{b}=1-r$ with the corresponding remainder term $r$ of order -1 , and let $\tilde{r}$ be any symbol of order -1 within the same class having the asymptotic expansion

$$
\tilde{r} \underset{(V)}{\sim} \sum_{k=1}^{\infty} \underbrace{r \# \ldots \# r}_{k \text {-times }} .
$$

At this stage of the proof it is important to having achieved the result about asymptotic completeness of all symbol classes involved, i. e., that it is always possible to find symbols within the classes having a prescribed asymptotic expansion; see Remark 3.8 and Remark 5.4.

It is evident from the asymptotic expansion that $(1-r) \#(1+\tilde{r})-1$ is of order $-\infty$, and, consequently, $a \#(\tilde{b} \#(1+\tilde{r}))-1$ is of order $-\infty$. Hence we have constructed a symbol $b_{(R)}:=\tilde{b} \#(1+\tilde{r})$ that fulfills the assertions in b) and ii) with respect to the Leibniz-product from the right. Analogously, we can construct a parametrix 
$b_{(L)}$ from the left, but $b_{(R)}$ and $b_{(L)}$ differ only by a term of order $-\infty$, which follows immediately from considering the product $b_{(L)} \# a \# b_{(R)}$, keeping in mind the ideal property of the symbol classes of order $-\infty$. Thus we may choose either $b:=b_{(L)}$ or $b:=b_{(R)}$, and the desired assertion in b) or ii) follows.

6.9 Remark. As usual, a (Volterra) Mellin operator op ${ }_{M}^{\gamma}(a)(\xi)$ within the general or holomorphic calculus is called elliptic or parabolic if its symbol $a(r, \xi, z)$ has this property.

From the considerations about Mellin quantization in Section 5.2 we see, however, that modulo smoothing (Volterra) operators the operator op ${ }_{M}^{\gamma}(a)(\xi)$ has a representation as a standard (Kohn-Nirenberg quantized) pseudodifferential operator on the half-axis with a degenerate operator-valued (Volterra) symbol (see Theorem 5.9). More precisely, we have op ${ }_{M}^{\gamma}(a)(\xi) \equiv \mathrm{op}_{r}(b)(\xi)$ modulo smoothing parameter-dependent (Volterra) operators, where $b(r, \xi, \tau)=\tilde{a}(r, \xi, r \tau)$ with

$$
\tilde{a}(r, \xi, \tau) \in\left\{\begin{array}{l}
C^{\infty}\left(\mathbb{R}_{+}, S_{(c l)}^{\mu ; \ell}\left(\mathbb{R}^{n} \times \mathbb{R} ; E, \tilde{E}\right)\right) \\
C^{\infty}\left(\mathbb{R}_{+}, S_{V(c l)}^{\mu ; \ell}\left(\mathbb{R}^{n} \times \mathbb{H} ; E, \tilde{E}\right)\right) .
\end{array}\right.
$$

Moreover, in case of classical symbols $a(r, \xi, z)$, the identity

$$
a_{(\mu)}\left(r, \xi, \frac{1}{2}-\gamma-i \tau\right)=\tilde{a}_{(\mu)}(r, \xi, \tau)=b_{(\mu)}\left(r, \xi, \frac{1}{r} \tau\right)
$$

is valid for the principal symbols. In particular, using Proposition 6.3 and Theorem 6.6 , this induces the notions of ellipticity and parabolicity for degenerate symbols $b(r, \xi, \tau)$, and Theorem 6.8 may be interpreted also as a result about the existence of (Volterra) parametrices to elliptic and parabolic standard pseudodifferential operators on the half-axis built upon degenerate operator-valued symbols.

\section{References}

[1] M. S. Agranovich and M. I. Vishik. Elliptic problems with a parameter and parabolic problems of general form. Russ. Math. Surv., 19(3):53-157, 1964. Engl. transl. of Usp. Mat. Nauk. 19(3):53-161, 1964.

[2] S. Behm. Pseudo-differential Operators with Parameters on Manifolds with Edges. PhD thesis, Universität Potsdam, 1995.

[3] L. Boutet de Monvel. Boundary problems for pseudo-differential operators. Acta Math., 126:11-51, 1971.

[4] Th. Buchholz. Parabolische Pseudodifferentialoperatoren mit operatorwertigen Symbolen. PhD thesis, Universität Potsdam, 1996. 
[5] Th. Buchholz and B.-W. Schulze. Anisotropic edge pseudo-differential operators with discrete asymptotics. Math. Nachr., 184:73-125, 1997.

[6] Th. Buchholz and B.-W. Schulze. Volterra operators and parabolicity. Anisotropic pseudo-differential operators. Preprint 98/11, Institute for Mathematics, Potsdam, 1998.

[7] Ch. Dorschfeldt. Algebras of Pseudo-differential Operators near Edge and Corner Singularities, volume 102 of Math. Research. Wiley-VCH, Berlin, 1998.

[8] Ch. Dorschfeldt, U. Grieme, and B.-W. Schulze. Pseudo-differential calculus in the Fourier-Edge approach on non-compact manifolds. In M. Demuth, E. Schrohe, B.-W. Schulze, and J. Sjöstrand, editors, Spectral Theory, Microlocal Analysis, Singular Manifolds, volume 14 of Math. Topics, Advances in Partial Differential Equations, pages 249-299, Akademie Verlag, Berlin, 1997.

[9] Ch. Dorschfeldt and B.-W. Schulze. Pseudo-differential operators with operator-valued symbols in the Mellin-Edge approach. Ann. Global Anal. Geom., 12:135-171, 1994.

[10] Ju. V. Egorov and B.-W. Schulze. Pseudo-differential Operators, Singularities, Applications, volume 93 of Operator Theory: Advances and Applications. Birkhäuser Verlag, Basel-Boston-Berlin, 1997.

[11] S. D. Ejdel'man and N. V. Zhitarashu. Parabolic Boundary-Value Problems, volume 101 of Operator Theory: Advances and Applications. Birkhäuser Verlag, Basel-Boston-Berlin, 1998.

[12] G. I. Eskin. Boundary Value Problems for Elliptic Pseudodifferential Equations, volume 52 of Math. Monographs. Amer. Math. Soc., Providence, Rhode Island, 1980. Engl. transl. of Nauka, Moskva, 1973.

[13] J. B. Gil. Heat Trace Asymptotics for Cone Differential Operators. PhD thesis, Universität Potsdam, 1998.

[14] J. B. Gil, B.-W. Schulze, and J. Seiler. Holomorphic operator-valued symbols for edge-degenerate pseudo-differential operators. In M. Demuth and B.-W. Schulze, editors, Differential Equations, Asymptotic Analysis, and Mathematical Physics, volume 100 of Math. Research, pages 113-137, Akademie Verlag, Berlin, 1997.

[15] J. B. Gil, B.-W. Schulze, and J. Seiler. Cone pseudodifferential operators in the edge symbolic calculus. Osaka J. Math., 37:221-260, 2000.

[16] G. Grubb. Functional Calculus of Pseudodifferential Boundary Problems, volume 65 of Progress in Mathematics. Birkhäuser Verlag, Basel-BostonBerlin, second edition, 1996. 
[17] T. Hirschmann. Functional analysis in cone and edge Sobolev spaces. Ann. Glob. Anal. Geom., 8(2):167-192, 1990.

[18] L. Hörmander. Pseudo-differential operators. Comm. Pure Appl. Math., 18:501-517, 1965.

[19] C. Iwasaki. The fundamental solution for parabolic pseudo-differential operators of parabolic type. Osaka J. Math., 14:569-592, 1977.

[20] P. Jeanquartier. Transformation de Mellin et développement asymptotiques. L'Enseignement mathématique, 25:285-308, 1979.

[21] J. J. Kohn and L. Nirenberg. An algebra of pseudo-differential operators. Comm. Pure Appl. Math., 18:269-305, 1965.

[22] V. A. Kondrat'ev. Boundary problems for elliptic equations in domains with conical or angular points. Trans. Moscow Math. Soc., 16:227-313, 1967.

[23] T. Krainer. On the calculus of pseudodifferential operators with an anisotropic analytic parameter. In preparation.

[24] T. Krainer. Parabolic pseudodifferential operators and long-time asymptotics of solutions. PhD thesis, Universität Potsdam, 2000.

[25] T. Krainer and B.-W. Schulze. Long-time asymptotics with geometric singularities in the spatial variables. Preprint 2000/17, Institute of Mathematics, Potsdam, 2000.

[26] T. Krainer and B.-W. Schulze. On the inverse of parabolic systems of partial differential equations of general form in an infinite space-time cylinder. Preprint 2001/14-16, Institute of Mathematics, Potsdam, 2001. To appear in Advances in Partial Differential Equations.

[27] H. Kumano-go. Pseudo-differential Operators. The MIT Press, Cambridge (MA), London, 1981.

[28] J. E. Lewis and C. Parenti. Pseudodifferential operators of Mellin type. Comm. Partial Differential Equations, 5:477-544, 1983.

[29] R. B. Melrose and G. A. Mendoza. Elliptic operators of totally characteristic type. Preprint, MSRI, 1983.

[30] A. Piriou. Une classe d'opérateurs pseudo-différentiels du type de Volterra. Ann. Inst. Fourier, Grenoble, 20(1):77-94, 1970.

[31] A. Piriou. Problèmes aux limites généraux pour des opérateurs différentiels paraboliques dans un domaine borné. Ann. Inst. Fourier, Grenoble, 21(1):59$78,1971$. 
[32] S. Rempel and B.-W. Schulze. Index Theory of Elliptic Boundary Problems. Akademie Verlag, Berlin, 1982.

[33] S. Rempel and B.-W. Schulze. Parametrices and boundary symbolic calculus for elliptic boundary problems without the transmission property. Math. Nachr., 105:45-149, 1982.

[34] H. H. Schaefer. Topological Vector Spaces, volume 3 of Graduate Texts in Mathematics. Springer Verlag, Berlin-Heidelberg-New York, 1971.

[35] E. Schrohe and B.-W. Schulze. Boundary value problems in Boutet de Monvel's calculus for manifolds with conical singularities I. In Pseudo-differential Calculus and Mathematical Physics, Advances in Partial Differential Equations, pages 97-209, Akademie Verlag, Berlin, 1994.

[36] E. Schrohe and B.-W. Schulze. Boundary value problems in Boutet de Monvel's calculus for manifolds with conical singularities II. In Boundary Value Problems, Schrödinger Operators, Deformation Quantization, Advances in Partial Differential Equations, pages 70-205, Akademie Verlag, Berlin, 1995.

[37] B.-W. Schulze. Mellin expansions of pseudo-differential operators and conormal asymptotics of solutions. In Proc. Oberwolfach 1986, volume 1256 of Lecture Notes in Mathematics, Springer Verlag, Berlin-Heidelberg-New York, 1987.

[38] B.-W. Schulze. Pseudo-differential operators on manifolds with edges. In H. Triebel et al., editors, Symposium 'Partial Differential Equations' 1988, Holzhau (Germany), volume 112 of Teubner Texte zur Mathematik, pages 259-287, B. G. Teubner, Stuttgart, 1989.

[39] B.-W. Schulze. Mellin representations of pseudo-differential operators on manifolds with corners. Ann. Glob. Anal. Geom., 8(3):261-297, 1990.

[40] B.-W. Schulze. Pseudo-differential Operators on Manifolds with Singularities. North Holland, Amsterdam, 1991.

[41] B.-W. Schulze. The Mellin pseudo-differential calculus on manifolds with corners. In H. Triebel et al., editors, Symposium 'Analysis on Manifolds with Singularities' 1990, Breitenbrunn (Germany), volume 131 of Teubner Texte zur Mathematik, pages 208-289, B. G. Teubner, Stuttgart, 1992.

[42] B.-W. Schulze. Pseudo-differential Boundary Value Problems, Conical Singularities, and Asymptotics, volume 4 of Math. Topics. Akademie Verlag, Berlin, 1994.

[43] B.-W. Schulze. Boundary Value Problems and Singular Pseudo-differential Operators. J. Wiley, Chichester, 1998. 
[44] B.-W. Schulze. Operator algebras with symbol hierarchies on manifolds with singularities. In J. Gil, D. Grieser, and M. Lesch, editors, Approaches to Singular Analysis, Advances in Partial Differential Equations, Oper. Theory Adv. Appl., pages 167-207, Birkhäuser Verlag, Basel-Boston-Berlin, 2001.

[45] R. T. Seeley. Complex powers of an elliptic operator, volume 10 of Amer. Math. Soc. Proc. Symp. Pure Math., pages 288-307. Amer. Math. Soc., Providence, Rhode Island, 1967.

[46] J. Seiler. Pseudodifferential Calculus on Manifolds with Non-Compact Edges. PhD thesis, Universität Potsdam, 1997.

[47] J. Seiler. Continuity of edge and corner pseudodifferential operators. Math. Nachr., 205:163-182, 1999.

[48] M. A. Shubin. Pseudodifferential Operators and Spectral Theory. Springer Verlag, Berlin-Heidelberg-New York, 1987.

[49] V. A. Solonnikov. Boundary value problems for linear parabolic systems of differential equations in general form. Proc. Steklov Inst. Math., 83, 1965. Engl. transl. of Tr. Mat. Inst. Steklova 83, 1965.

[50] A. Unterberger. The calculus of pseudo-differential operators of Fuchs type. Comm. Partial Differential Equations, 9:1179-1236, 1984. 